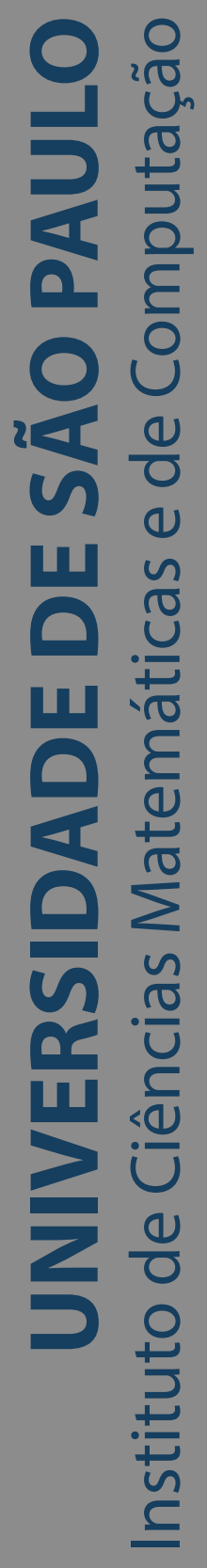

\title{
Parallelizable semidynamical systems
}

\section{Tiago Alves Pacifico}

Tese de Doutorado do Programa de Pós-Graduação em Matemática (PPG-Mat) 

Data de Depósito:

Assinatura:

\section{Tiago Alves Pacifico}

\section{Parallelizable semidynamical systems}

Thesis submitted to the Instituto de Ciências Matemáticas e de Computação - ICMC-USP - in accordance with the requirements of the Mathematics Graduate Program, for the degree of Doctor in Science. EXAMINATION BOARD PRESENTATION COPY

Concentration Area: Mathematics

Advisor: Prof. Dr. Everaldo de Mello Bonotto

USP - São Carlos

September 2021 
Ficha catalográfica elaborada pela Biblioteca Prof. Achille Bassi e Seção Técnica de Informática, ICMC/USP, com os dados inseridos pelo(a) autor(a)

Pacífico, Tiago Alves

Parallelizable semidynamical systems / Tiago

Alves Pacífico; orientador Everaldo de Mello

Bonotto. -- São Carlos, 2021.

$102 \mathrm{p}$.

Tese (Doutorado - Programa de Pós-Graduação em Matemática) -- Instituto de Ciências Matemáticas e de Computação, Universidade de São Paulo, 2021.

1. Semidynamical Systems. 2. Impulsive Semidynamical systems. 3. Sections. 4. Parallelizable Systems. 5. Dispersive Systems. I. Bonotto, Everaldo de Mello, orient. II. Título.

Bibliotecários responsáveis pela estrutura de catalogação da publicação de acordo com a AACR2: Gláucia Maria Saia Cristianini - CRB - 8/4938 Juliana de Souza Moraes - CRB - 8/6176 


\section{Tiago Alves Pacifico}

\section{Sistemas semidinâmicos paralelizáveis}

Tese apresentada ao Instituto de Ciências Matemáticas e de Computação - ICMC-USP, como parte dos requisitos para obtenção do título de Doutor em Ciências - Matemática. EXEMPLAR $D E D E F E S A$

Área de Concentração: Matemática

Orientador: Prof. Dr. Everaldo de Mello Bonotto

\section{USP - São Carlos}

Setembro de 2021 

To my mother and my father, they deserve the good things. 

Certainly, I will not be able to express in words all the greatness I feel for the people who have helped me throughout my studies, but I will try as far as I can. In this way, I apologize if you feel unfair.

First of all, I am grateful to God for giving me health, strength and resilience to face each difficulty that was imposed on me.

I am grateful to my advisor Professor Dr. Everaldo de Mello Bonotto, who sheltered me from the beginning, guided me throughout the research and encouraged me to overcome all obstacles on this journey.

I would like to thank my family who always supported me, encouraged me, and set me on the path of education from an early age. In special, I am grateful to my mother and my father for all the efforts they have made so that I could fight for my dreams. I thank my brothers who always helped when needed.

I thank all my friends who make this journey less arduous. In particular, to all the friends that I knew during my PhD.

I also thank my girlfriend for all her love, patience and affection with me.

Finally, I thank to CAPES for the financial support through the process PROEX-9725248/D. 

"Seek not to understand that you may believe, but believe that you may understand."

(St. Augustine) 



\section{RESUMO}

PACÍFICO, T. A. Sistemas semidinâmicos paralelizáveis. 2021. 102 p. Tese (Doutorado em Ciências - Matemática) - Instituto de Ciências Matemáticas e de Computação, Universidade de São Paulo, São Carlos - SP, 2021.

Neste trabalho, introduzimos o conceito de paralelizabilidade para sistemas semidinâmicos. Estudamos propriedades topológicas de sistemas semidinâmicos paralelizáveis contínuos e impulsivos. Duas relações importantes são investigadas tanto no estudo de sistemas semidinâmicos contínuos quanto no estudo de sistemas semidinâmicos com impulsos: a relação entre sistemas paralelizáveis e a existência de seções e a relação entre sistemas paralelizáveis e sistemas dispersivos. Além disso, apresentamos um estudo de paralelizabilidade na região de atração fraca de um conjunto compacto, fortemente invariante e assintoticamente estável.

Os resultados apresentados nesta tese deram origem aos seguintes artigos:

(1) Recursive properties on semidynamical systems: parallelizability and dispersiveness. Veja (BONOTTO; PACIFICO, 2021b).

(2) Parallelizable impulsive semidynamical systems. Veja (BONOTTO; PACIFICO, 2021a).

Palavras-chave: Sistemas Semidinâmicos, Sistemas Semidinâmicos Impulsivos, Seções, Sistemas Paralelizáveis, Sistemas Dispersivos. 



\section{ABSTRACT}

PACÍFICO, T. A. Parallelizable semidynamical systems. 2021. 102 p. Tese (Doutorado em Ciências - Matemática) - Instituto de Ciências Matemáticas e de Computação, Universidade de São Paulo, São Carlos - SP, 2021.

In this work, we introduce the concept of parallelizability for semidynamical systems. We study topological properties of impulsive and continuous parallelizable semidynamical systems. Two important relationships are investigated both in the study of continuous semidynamical systems and in the study of impulsive semidynamical systems: the relationship between parallelizable systems and the existence of sections and the relationship between parallelizable systems and dispersive systems. In addition, we present a study of parallelizability in the region of weak attraction of a compact, strongly invariant and asymptotically stable set.

The new results presented in this thesis gave rise to the following articles:

(1) Recursive properties on semidynamical systems: parallelizability and dispersiveness. See (BONOTTO; PACIFICO, 2021b).

(2) Parallelizable impulsive semidynamical systems. See (BONOTTO; PACIFICO, 2021a).

Keywords: Semidynamical Systems, Impulsive Semidynamical Systems, Sections, Parallelizable Systems, Dispersive Systems. 



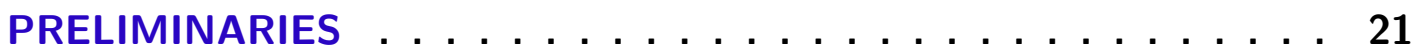

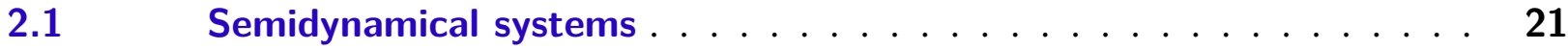

$2.2 \quad$ Impulsive semidynamical systems . . . . . . . . . . . . 26

2.2.1 Continuity of the impact function $\phi \ldots \ldots \ldots 32$

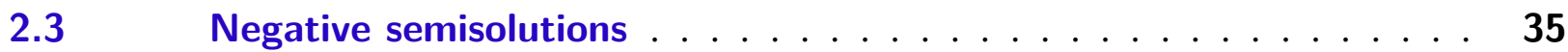

2.3.1 Negative semisolutions for semidynamical systems . . . . . . . . . 35

2.3.2 Negative semisolutions for impulsive semidynamical systems . . . . 36

3 PARALLELIZABLE SEMIDYNAMICAL SYSTEMS $\ldots \ldots . \ldots$

$3.1 \quad$ Parallelizable semidynamical systems . . . . . . . . . . 41

$3.2 \quad$ Parallelizability versus sections . . . . . . . . . . . . 50

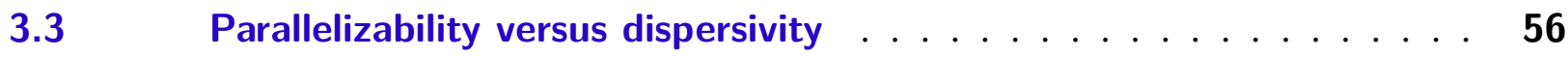

$3.4 \quad$ Parallelizability versus negative semisolutions $\ldots \ldots \ldots \ldots$

4 PARALLELIZABLE IMPULSIVE SEMIDYNAMICAL SYSTEMS . . . 61

$4.1 \quad$ Continuity of the impact function $\phi \ldots \ldots \ldots$. . . . . . .

$4.2 \quad$ Parallelizable impulsive semidynamical systems . . . . . . . . . 68

$4.3 \quad$ Parallelizability versus sections . . . . . . . . . . . . 74

$4.4 \quad$ Parallelizability versus dispersivity . . . . . . . . . . . 78

5 PARALLELIZABILITY AND THE REGION OF WEAK ATTRACTION 85

$5.1 \quad$ Parallelizable impulsive region of weak attraction . . . . . . 85

$5.2 \quad$ Application in autonomous differential equations . . . . . . . 93

BIBLIOGRAPHY . . . . . . . . . . . . . . . . . . . 99 

CHAPTER

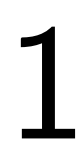

1

INTRODUCTION

The study of parallelizable dynamical systems started with H. Whitney in the paper (WHITNEY, 1932). Under certain conditions, Whitney proved that a parallelizable dynamical system is homeomorphic to a family of parallel lines. Besides that, Whitney concluded that the existence of a parallelizable dynamical system is equivalent to the existence of a section with section map continuous.

In 1960, J. Dugundji and H. A. Antosiewicz proved the equivalence between parallelizable dynamical systems and dispersive dynamical systems on separable locally compact metric spaces. This study is presented in the paper (ANTOSIEWICZ; DUGUNDJI, 1960). This equivalence also was investigated by Nemytskii and Stepanov in (NEMYTSKII; STEPANOV, 1989).

In (URA; EGAWA, 1973), J. Egawa together with T. Ura, established the study of isomorphisms between two parallelizable dynamical systems. In particular, by considering a topological space $X$, they found how many distinct ways it is possible to write $X=W \times \mathbb{R}, W \subset X$. This result together with others from the classical topology, showed that in $\mathbb{R}^{n}, n \geqslant 4$, the number of parallelizable dynamical systems which are not isomorphic to each other is not enumerable.

Recently, J. A. Souza et al., (PACIFICO; SOUZA; TOZATTI, 2017), demonstrated that a parallelizable system is equivalent to a trivial principal bundle with fiber homeomorphic to a section.

As presented in (BHATIA; SZEGÖ, 2002), parallelizability implies the absence of stationary points, periodic points, non-wandering points and of Poisson stable points. 
However, the theory of parallelizability has not yet been studied in the context of semidynamical systems (one-sided dynamical systems). Motivated by this fact, we propose in this work to introduce a notion of parallelizability for semidynamical systems and to study their topological properties.

On the other hand, many real world problems can experience abrupt external forces which can change completely their dynamics, for example, the drug distribution and bursting rhythm models in medicine, optimal control in economics and growth population problems. The theory of impulsive differential equations has been successfully used to describe a variety of phenomena that present abrupt perturbations. See, for instance, (CHURCH; LIU, 2019),(GUO; SHEN; GUI, 2021), (LUO; WANG, 2021), (SUGIE; ISHIHARA, 2021), (VADIVOO et al., 2020) and (YU ; TANG, 2002). Among the different types of impulses, we highlight the impulses that vary in time which are more attractive due to their complexity and applicability in real phenomena. The study of semidynamical systems subject to impulses at variable times started with Kaul in the early 1990's, see (KAUL, 1990; KAUL, 1991). Thereafter, many authors have contributed to the development of this theory, for instance, see (AFONSO; BONOTTO; JIMENEZ, 2015), (BONOTTO et al., 2015), (BONOTTO; GRULHA JR, 2010), (BONOTTO; FEDERSON, 2007), (BONOTTO, 2007), (CIESIELSKI, 2004a), (CIESIELSKI, 2004b), (DASHKOVSKIY et al., 2018), (DING; DUAN; PAN, 2020), (DING; DING, 2017), (DING, 2014), (KAPUSTYAN; PERESTYUK, 2015) and (KAPUSTYAN; PERESTYUK, 2016). Thus, we also propose to introduce a notion of parallelizability in the context of impulsive semidynamical systems.

In what follows, we describe a summary of each chapter of this work.

In Chapter 2, we present the basis of the theories of continuous semidynamical systems and impulsive semidynamical systems. Section 2.1 is devoted to the basic concepts of the theory of continuous semidynamical systems, which we just call as semidynamical systems. Some examples are presented in order to show a phenomenon that can occur in the study of one-sided dynamical systems called "the absence of negative uniqueness", see Example 2.1.2 for instance. In Section 2.2 , we exhibit the theory of impulsive semidynamical systems which describes the evolution of systems where the continuous development of a process is interrupted by abrupt changes of state. In Example 2.2.1, we show that a simple perturbation can drastically changes the structure of a system. As part of this section, we discuss in Subsection 2.2.1, the continuity of the impact function (defined in Definition 2.2.4) which plays an important role to ensure the well behavior of the trajectories of impulsive semidynamical systems. The continuity of this function is established by using tube 
conditions. However, the tube conditions are not easy to verify, specially in infinite dimension spaces. In this way, we propose another condition to obtain the continuity of the impact function and this will be studied in Chapter 4. In Section 2.3, we present an introduction to the theory of negative semisolutions, since this theory will be useful in Section 3.4 from Chapter 3 and in Chapter 5. In Subsection 2.3.1, we exhibit basic facts of negative semisolutions for semidynamical systems, and, in Subsection 2.3.2, we dedicate to construct an impulsive negative semisolution.

In Chapter 3, we begin with the presentation of our contribution by introducing the theory of parallelizable semidynamical systems. Section 3.1 characterizes the semidynamical systems that are parallelizable (see Definition 3.1.2). In Definition 3.1.3, we introduce the concept of a section for semidynamical systems and we show that it is possible to define a function called "section function" associated to this section. Some auxiliary results concerning sections are presented in Lemma 3.1.3, Lemma 3.1.4 and in Lemma 3.1.5. Section 3.2 explores the relationship between parallelizable semidynamical systems and the existence of sections. In Theorem 3.2.1, we show that a parallelizable semidynamical system admits a section with continuous function section. The converse of this fact does not hold in general. In this way, we present in Theorem 3.2.2 and in Corollary 3.2.2, sufficient conditions to obtain the converse of Theorem 3.2.1. In Section 3.3, we study the relationship between parallelizable semidynamical systems and dispersive semidynamical systems. In Theorem 3.3.1, we show that every parallelizable semidynamical system is dispersive. The Example 3.3.1 reveals that the converse of Theorem 3.3.1 also is not true in general. But in Theorem 3.3.2 and in Corollary 3.3.1, we provide sufficient conditions for the validity of the converse of Theorem 3.3.1. We end this chapter with Section 3.4. We provide sufficient conditions for a semidynamical system to be parallelizable via negative semisolutions, see Theorem 3.4.1.

In Chapter 4, we dedicate to the study of parallelizable impulsive semidynamical systems. As commented earlier in the description of Subsection 2.2.1, we start Chapter 4 with Section 4.1, where we introduce a new condition called "condition (C)" (see Definiton 4.1.1). If an impulsive set satisfies the condition (C), then we prove in Theorem 4.1.1 that the impact function is continuous outside of the impulsive set. Besides that, we improved some results presented in the literature on convergence by using the condition (C). These results are exhibited in Lemma 4.1.3, Lemma 4.1.4, Corollary 4.1.2 and Lemma 4.1.5. In Section 4.2, we introduce the concepts of parallelizability and section for impulsive semidynamical systems. Section 4.3 deals with the relationship between parallelizable impulsive semidynamical systems and the existence of sections. Theorem 4.3.1 says that every parallelizable impulsive semidynamical systems admits a section with associated section 
function continuous outside of the impulsive set. As done in the continuous case in Chapter 3 , we provide in Theorem 4.3.2 sufficient conditions for the validity of the converse of Theorem 4.3.1. In Section 4.4, we analyze the relationship between parallelizable impulsive semidynamical systems and dispersive impulsive semidynamical systems. Theorem 4.4.1 gives us sufficient conditions for a parallelizable impulsive semidynamical systems to be dispersive. On the other hand, Theorems 4.4.2 and 4.4.3 give sufficient conditions to obtain the converse of Theorem 4.4.1.

Chapter 5 is devoted to present sufficient conditions for the region of weak attraction of a compact, strongly invariant and asymptotically stable set to be parallelizable. The results are obtained for impulsive semidynamical systems. As a consequence, we obtain the same results for continuous semidynamical systems. In Section 5.1, we start by presenting some auxiliary results. In Theorem 5.1.3, under some conditions, we prove that the region of weak attraction of a compact, strongly invariant and asymptotically stable set excluding it and the impulsive set is parallelizable. In Section 5.2, we present an application in autonomous differential equations. We consider an impulsive ordinary differential equation of type

$$
\left\{\begin{array}{l}
x^{\prime}=f(x), \\
I: M \rightarrow \mathbb{R}^{n},
\end{array}\right.
$$

where $M$ is an impulsive set, and we define the concepts of parallelizability and dispersiveness for this impulsive equation (see Definition 5.2.1). We show that the impulsive differential equation (1.0.1) does not admit bounded solutions with closure contained in a parallelizable region, see Proposition 5.2.1 and Corollary 5.2.1. In Theorem 5.2.1, by using Lyapunov functions, we provide sufficient conditions for the impulsive differential equation (1.0.1) to be parallelizable. 
CHAPTER

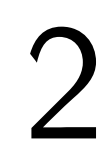

(1)

\section{PRELIMINARIES}

The present chapter deals with basic concepts and some results from the theories of continuous and impulsive semidynamical systems. Section 2.1 is based on the book (BHATIA; HAJEK, 1969). Section 2.2 was written based on the papers (BONOTTO; FEDERSON, 2007; BONOTTO, 2007; BONOTTO et al., 2015; CIESIELSKI, 2004a; KAUL, 1990). Section 2.3 is divided into two subsections. Subsection 2.3.1 was based on (BHATIA; HAJEK, 1969; BISTROŃ, 2003; BISTROŃ, 2005) and Subsection 2.3.2 was based on (AFONSO; BONOTTO; JIMENEZ, 2015).

Throughout this text, we will denote by $\mathbb{R}$ the set of all real numbers, $\mathbb{R}_{+}=\{x \in \mathbb{R}: x \geqslant 0\}$, $\mathbb{R}_{-}=\{x \in \mathbb{R}: x \leqslant 0\}$ and by $\mathbb{R}^{n}$ the real $n$-dimensional euclidean space. The set of all natural numbers will be denoted by $\mathbb{N}=\{1,2, \ldots\}$ and $\mathbb{N}_{0}=\mathbb{N} \cup\{0\}$.

\subsection{Semidynamical systems}

Let $(X, d)$ be a metric space. Given $\delta>0$ and $x \in X$, by $B(x, \delta)$ we mean the open ball with center at $x$ and radius $\delta$. The $\delta$ neighborhood of $A$ is represented by

$$
B(A, \delta)=\{x \in X: d(x, A)<\delta\}
$$

where $d(x, A)=\inf \{d(x, y): y \in A\}$.

Definition 2.1.1. The Hausdorff semidistance between two nonempty bounded subsets $A$ and $B$ 
of $X$, is given by

$$
d_{H}(A, B)=\sup \{d(a, B): a \in A\}
$$

Next, we present the concept of semidynamical systems.

Definition 2.1.2. A triple $\left(X, \pi, \mathbb{R}_{+}\right)$is called a continuous semidynamical system on $X$, if the mapping $\pi: X \times \mathbb{R}_{+} \rightarrow X$ is continuous on $X \times \mathbb{R}_{+}$and satisfies the following conditions:

(i) $\pi(x, 0)=x$ for all $x \in X$;

(ii) $\pi(\pi(x, t), s)=\pi(x, t+s)$ for all $x \in X$ and $t, s \in \mathbb{R}_{+}$.

Condition $(i)$ is known as the identity axiom and condition (ii) is known as the semigroup axiom. The mapping $\pi: X \times \mathbb{R}_{+} \rightarrow X$ is called a phase map and $X$ is called the phase space.

Remark 2.1.1. From now on, we will drop the term "continuous" and we will simply call $\left(X, \pi, \mathbb{R}_{+}\right)$a semidynamical system.

Remark 2.1.2. In Definition 2.1.2, if we replace $\mathbb{R}_{+}$by $\mathbb{R}$ then the triple $(X, \pi, \mathbb{R})$ is called a dynamical system on $X$. Sometimes we will use the term semiflow (flow) instead of semidynamical system (dynamical system).

For each $x \in X$, the continuous map $\pi_{x}: \mathbb{R}_{+} \rightarrow X$ given by $\pi_{x}(t)=\pi(x, t)$ is called the motion through $x$. And, for each $t \in \mathbb{R}_{+}$, the continuous map $\pi_{t}: X \rightarrow X$ given by $\pi_{t}(x)=\pi(x, t)$ is called a transition.

Next, we present a classical example from autonomous differential equations. The reader may consult (BHATIA; HAJEK, 1969, Example 0.3) for more details.

Example 2.1.1. Let $f: \mathbb{R}^{n} \rightarrow \mathbb{R}^{n}$ be a function and consider the autonomous differential equation

$$
\frac{d x}{d t}=f(x)
$$

Assume that $f$ satisfies sufficient conditions such that for each $x \in \mathbb{R}^{n}$, there exists a unique solution $\phi(t, x)$ of the ordinary differential equation (2.1.1) passing through $x$ at time zero, i.e., $\phi(0, x)=x$. Using the uniqueness of solutions, we conclude that

$$
\phi(t, \phi(s, x))=\phi(t+s, x),
$$


for all $x \in \mathbb{R}^{n}$ and $t, s \in \mathbb{R}_{+}$. Hence, the map $\pi: \mathbb{R}^{n} \times \mathbb{R}_{+} \rightarrow \mathbb{R}^{n}$ given by $\pi(x, t)=\phi(t, x)$ defines a semidynamical system on $\mathbb{R}^{n}$.

Definition 2.1.3. The positive trajectory of $x \in X$ in $\left(X, \pi, \mathbb{R}_{+}\right)$is defined by

$$
\pi^{+}(x)=\left\{\pi(x, t): t \in \mathbb{R}_{+}\right\}
$$

Furthermore, given $A \subset X$ and $\Delta \subset \mathbb{R}_{+}$, we define

$$
\pi^{+}(A)=\bigcup_{x \in A} \pi^{+}(x) \quad \text { and } \quad \pi(A, \Delta)=\bigcup_{x \in A, t \in \Delta} \pi(x, t)
$$

In the study of semidynamical systems, a phenomenon that can occur is the absence of negative uniqueness, that is, it is possible that there are $x, y \in X, x \neq y$, such that $\pi(x, t)=\pi(y, t)$ for some $t>0$. This phenomenon is illustrated in the next example.

Example 2.1.2. Let $X=\{(x, 0): x \in \mathbb{R}\} \bigcup\{(1, y): 0<y \leqslant 1\} \subset \mathbb{R}^{2}$ and $\left(X, \pi, \mathbb{R}_{+}\right)$be a semidynamical system, where $\pi: X \times \mathbb{R}_{+} \rightarrow X$ is defined by

$$
\pi((x, 0), t)=(t+x, 0) \quad \text { if } \quad x \in \mathbb{R}, t \in \mathbb{R}_{+},
$$

and

$$
\pi((1, y), t)= \begin{cases}(1, y-t) & \text { if } 0<y \leqslant 1,0 \leqslant t \leqslant y \\ (1+t-y, 0) & \text { if } 0<y \leqslant 1, t \geqslant y\end{cases}
$$

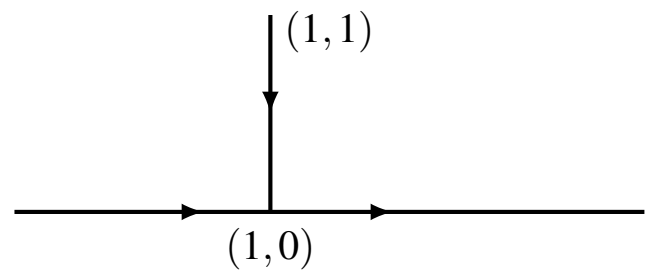

Figure 2.1: Positive trajectories of the $\operatorname{system}\left(X, \pi, \mathbb{R}_{+}\right)$.

Note that, for every $0<y \leqslant 1$, we have $\pi((1, y), t)=\pi((1-y, 0), t)$ whenever $t \geqslant y$.

In order to study the backward trajectory of a point in the phase space, we consider the past of a such point as defined in the next definition. 
Definition 2.1.4. Let $t \in \mathbb{R}_{+}$and $x \in X$. The past of $x$ at time $t$ with respect to a semidynamical system $\left(X, \pi, \mathbb{R}_{+}\right)$is represented by the set

$$
F(x, t)=\{y \in X: \pi(y, t)=x\}
$$

and, for $\Delta \subset[0, \infty)$ and $D \subset X$, we write

$$
F(D, \Delta)=\bigcup\{F(x, t): x \in D \text { and } t \in \Delta\}
$$

In Example 2.1.2, for each $x_{0} \geqslant 1$, the past of $\left(x_{0}, 0\right)$ at time $\lambda$ is given by

$$
F\left(\left(x_{0}, 0\right), \lambda\right)= \begin{cases}\left\{\left(x_{0}-\lambda, 0\right)\right\} & \text { if } 0 \leqslant \lambda \leqslant x_{0}-1, \\ \left\{\left(x_{0}-\lambda, 0\right),\left(1, \lambda-x_{0}+1\right)\right\} & \text { if } x_{0}-1<\lambda \leqslant x_{0}, \\ \left\{\left(x_{0}-\lambda, 0\right)\right\} & \text { if } \lambda>x_{0} .\end{cases}
$$

Definition 2.1.5. A set $A \subset X$ is called positively $\pi$-invariant if $\pi^{+}(A) \subset A$.

Note that the positive trajectory of a point $x \in X$ is positively $\pi$-invariant. The union and intersection of positively $\pi$-invariant sets are positively $\pi$-invariant, (BHATIA; HAJEK, 1969 , Lemma 3.3.2). The closure of a positively $\pi$-invariant set still positively $\pi$-invariant, see (BHATIA; HAJEK, 1969, Lemma 3.4.1).

Next, we present the concepts of stationary points and periodic points.

Definition 2.1.6. Let $\left(X, \pi, \mathbb{R}_{+}\right)$be a semidynamical system.

(i) A point $x \in X$ is said to be a stationary point if $\pi(x, t)=x$ for all $t \geqslant 0$.

(ii) A point $x \in X$ is said to be a periodic point if $\pi(x, t)=x$ for some $t>0$ and $x$ is not stationary. A positive trajectory $\pi^{+}(x), x \in X$, is called periodic if each point $y \in \pi^{+}(x)$ is periodic.

Definition 2.1.7 concerns the concepts of some "limit sets" in semidynamical systems.

Definition 2.1.7. Let $\left(X, \pi, \mathbb{R}_{+}\right)$be a semidynamical system and $A \subset X$.

(i) The positive limit set of $A$ is given by

$$
\begin{gathered}
\omega^{+}(A)=\left\{y \in X: \text { there are sequences }\left\{x_{n}\right\}_{n \in \mathbb{N}} \subset A \text { and }\left\{t_{n}\right\}_{n \in \mathbb{N}} \subset \mathbb{R}_{+}\right. \\
\text {such that } \left.t_{n} \stackrel{n \rightarrow \infty}{\longrightarrow \infty} \text { and } \pi\left(x_{n}, t_{n}\right) \stackrel{n \rightarrow \infty}{\longrightarrow} y\right\}
\end{gathered}
$$


(ii) The positive prolongational limit set of $A$ is given by

$$
\begin{gathered}
J^{+}(A)=\left\{y \in X: \text { there are sequences }\left\{x_{n}\right\}_{n \in \mathbb{N}} \subset X \text { and }\left\{t_{n}\right\}_{n \in \mathbb{N}} \subset \mathbb{R}_{+}\right. \\
\text {such that } \left.d\left(x_{n}, A\right) \stackrel{n \rightarrow \infty}{\longrightarrow} 0, t_{n} \stackrel{n \rightarrow \infty}{\longrightarrow} \infty \text { and } \pi\left(x_{n}, t_{n}\right) \stackrel{n \rightarrow \infty}{\longrightarrow} y\right\} .
\end{gathered}
$$

For each $x \in X$, we set $\omega^{+}(x)=\omega^{+}(\{x\})$ and $J^{+}(x)=J^{+}(\{x\})$. Note that $\omega^{+}(A) \subset J^{+}(A)$ for every subset $A \subset X$.

Lemma 2.1.1. (BHATIA; HAJEK, 1969, Theorems 5.4.2 and 6.3.2) Let $\left(X, \pi, \mathbb{R}_{+}\right)$be a semidynamical system and $A \subset X$. The sets $\omega^{+}(A)$ and $J^{+}(A)$ are closed in $X$ and positively $\pi$-invariant. Example 2.1.3. Let $X=\mathbb{R}^{2}$ be represented in its polar form, that is, for each $z \in \mathbb{R}^{2}$ there are $r \geqslant 0$ and $0 \leqslant \theta \leqslant 2 \pi$ such that $z=r e^{i \theta}$. The map given by

$$
\pi(z, t)= \begin{cases}r e^{i(\theta+t)} & \text { for } 0 \leqslant r \leqslant 1, t \geqslant 0 \\ (r-t) e^{i \theta} & \text { for } r>1,0 \leqslant t \leqslant r-1, \\ e^{i(\theta+t-r+1)} & \text { for } r>1, t>r-1,\end{cases}
$$

defines a semidynamical system on $\mathbb{R}^{2}$. The positive trajectories consist of rays for $r>1$ and circles for $0 \leqslant r \leqslant 1$, see Figura 2.2 .

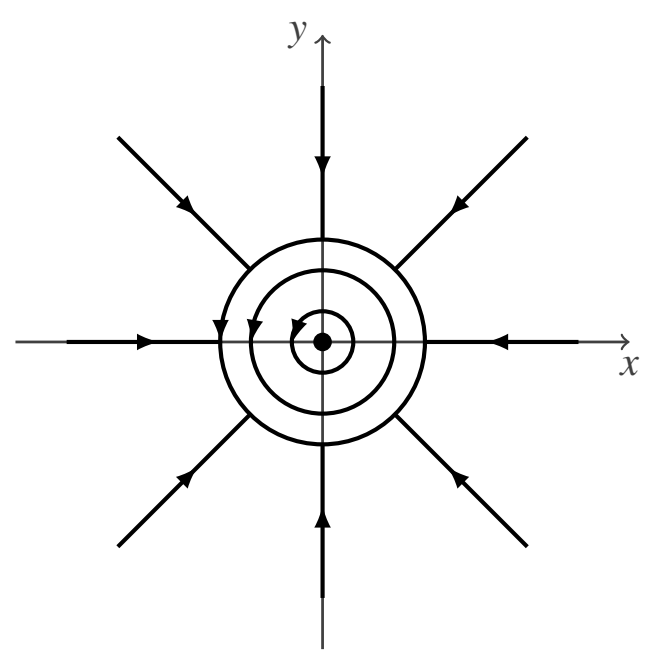

Figure 2.2: Positive trajectories of the semidynamical system (2.1.2).

Note that $r=0$ is a stationary point and for each $0<r \leqslant 1$ and $0 \leqslant \theta \leqslant 2 \pi$, the positive trajectory $\pi^{+}\left(r e^{i \theta}\right)$ is a periodic trajectory. For $r>1$ and $0 \leqslant \theta \leqslant 2 \pi$, we have

$$
\omega^{+}\left(r e^{i \theta}\right)=J^{+}\left(r e^{i \theta}\right)=S^{1}
$$


where $S^{1}$ is the unit circle. For $0 \leqslant r \leqslant 1$ and $0 \leqslant \theta \leqslant 2 \pi$,

$$
\omega^{+}\left(r e^{i \theta}\right)=J^{+}\left(r e^{i \theta}\right)=S^{r}
$$

where $S^{0}=\{(0,0)\}$ and $S^{r}=\left\{r e^{i \theta}: 0 \leqslant \theta \leqslant 2 \pi\right\}$ for $0<r \leqslant 1$.

Definition 2.1.8. The region of weak attraction of a set $A \subset X$ is defined by

$$
\begin{gathered}
P_{w}^{+}(A)=\left\{x \in X: \text { for every neighborhood } U \text { of } A \text {, there is a sequence }\left\{t_{n}\right\}_{n \in \mathbb{N}} \subset \mathbb{R}_{+},\right. \\
\left.\qquad t_{n} \stackrel{n \rightarrow \infty}{\longrightarrow} \infty \text {, such that } \pi\left(x, t_{n}\right) \in U \text { for every } n \in \mathbb{N}\right\} .
\end{gathered}
$$

Next, we present some concepts of stability.

Definition 2.1.9. A subset $A \subset X$ is said to be:

(i) orbitally $\pi$-stable, if for every neighborhood $U$ of $A$ there exists a positively $\pi$-invariant neighborhood $V$ of $A$ with $V \subset U$;

(ii) uniformly $\pi$-stable, if for every $\varepsilon>0$ there is $\delta=\delta(\varepsilon)>0$ such that

$$
\pi(B(A, \delta),[0, \infty)) \subset B(A, \varepsilon)
$$

(iii) weak $\pi$-attractor, if $P_{w}^{+}(A)$ is a neighborhood of $A$;

(iv) asymptotically $\pi$-stable, if it is both weak $\pi$-attractor and orbitally $\pi$-stable.

Example 2.1.4. In Example 2.1.3, let $A=\left\{r e^{i \theta}: 0 \leqslant r \leqslant 1\right.$ and $\left.0 \leqslant \theta \leqslant 2 \pi\right\}$. The set $A$ is orbitally $\pi$-stable. In fact, let $U$ be neighborhood of $A$. Thus, there is $\delta>0$ such that $B(A, \delta) \subset U$. Since $V=\pi\left(B(A, \delta), \mathbb{R}_{+}\right)$is a positively $\pi$-invariant neighborhood of $A$ with $V \subset U$, the result is proved.

The set $A$ is also weak $\pi$-attractor as $P_{w}^{+}(A)=\mathbb{R}^{2}$. Therefore, $A$ is asymptotically $\pi$-stable.

\subsection{Impulsive semidynamical systems}

The theory of impulsive semidynamical systems describes the evolution of systems where the continuous development of a process is interrupted by abrupt changes of state. V. Rozko started the study of impulsive semidynamical systems in the papers (ROZKO, 1972; ROZKO, 1975). 
In these works, V. Rozko considered systems where the impulses occur at fixed times. Just in the early 90's, the mathematician K. Saroop established the theory of impulsive semidynamical systems with impulses at variable times (see (KAUL, 1990; KAUL, 1991)). After that, several works have contributed to the solidification of this theory. The reader may consult (AFONSO; BONOTTO; JIMENEZ, 2015; BONOTTO; KALITA, 2020; BONOTTO; MESQUITA; PARREIRA, 2018; BONOTTO et al., 2016; BONOTTO et al., 2015; BONOTTO; GRULHA JR, 2010; BONOTTO; FEDERSON, 2007; BONOTTO; SOUTO, 2019; BONOTTO, 2007; CIESIELSKI, 2004a; CIESIELSKI, 2004b; DING; DUAN; PAN, 2020; DING; DING, 2017; DING, 2014; KAPUSTYAN; PERESTYUK, 2015; KAPUSTYAN; PERESTYUK, 2016).

We start by presenting the concepts of impulsive set and impulse function.

Definition 2.2.1. Let $\left(X, \pi, \mathbb{R}_{+}\right)$be a semidynamical system. A nonempty closed subset $M$ of $X$ is called an impulsive set, if for every $x \in M$ there exists $\varepsilon_{x}>0$ such that

$$
F\left(x,\left(0, \varepsilon_{x}\right)\right) \cap M=\emptyset \quad \text { and } \quad \pi\left(x,\left(0, \varepsilon_{x}\right)\right) \cap M=\emptyset .
$$

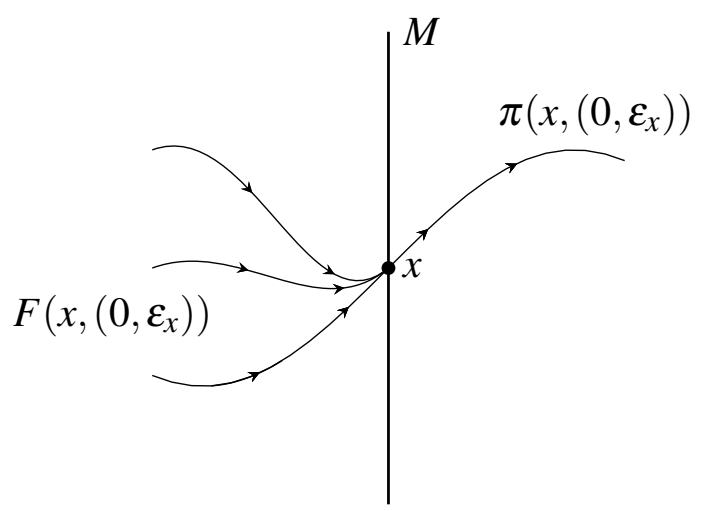

Figure 2.3: The trajectories cross the set $M$.

Definition 2.2.2. Let $\left(X, \pi, \mathbb{R}_{+}\right)$be a semidynamical system and $M \subset X$ be an impulsive set. A continuous function $I: M \rightarrow X$ is called an impulse function.

The next definition concerns the concept of an impulsive semidynamical system.

Definition 2.2.3. An impulsive semidynamical system (ISS for short), denoted by $(X, \pi, M, I)$, consists of a semidynamical system $\left(X, \pi, \mathbb{R}_{+}\right)$, an impulsive set $M \subset X$ and an impulse function $I: M \rightarrow X$. 
In the sequel, when we construct the positive impulsive trajectory, we explain the role of the impulse function $I: M \rightarrow X$. Before that, we present the concept of the impact function.

Definition 2.2.4. The impact function $\phi: X \rightarrow(0, \infty]$ is defined by

$$
\phi(x)= \begin{cases}s, & \text { if } \pi(x, s) \in M \text { and } \pi(x, t) \notin M \text { for } 0<t<s, \\ \infty, & \text { if } \pi(x, t) \notin M \text { for all } t>0 .\end{cases}
$$

Note that if $\left(\bigcup_{t>0} \pi(x, t)\right) \cap M \neq \emptyset$, for some $x \in X$, then the function $\phi(x)$ denotes the least positive time for which the positive trajectory of $x$ meets $M$. The point $\pi(x, \phi(x)), x \in X$, is called the impulsive point of $x$.

Definition 2.2.5. The positive impulsive trajectory of $x$ in $(X, \pi, M, I)$ is an $X$-valued function $\tilde{\pi}_{x}$ defined on a subset $[0, s)$ of $\mathbb{R}_{+}(s$ may be $\infty)$ whose description follows inductively: if $\phi(x)=\infty$, then

$$
\tilde{\pi}_{x}(t)=\pi(x, t) \quad \text { for all } \quad t \in \mathbb{R}_{+} .
$$

However, if $\phi(x)=s_{0}<\infty$ then $\pi\left(x, s_{0}\right)=x_{1} \in M$ and $\pi(x, t) \notin M$ for $0<t<s_{0}$. Thus, we define $\tilde{\pi}_{x}$ on $\left[0, s_{0}\right]$ by

$$
\tilde{\pi}_{x}(t)= \begin{cases}\pi(x, t), & 0 \leqslant t<s_{0} \\ x_{1}^{+}, & t=s_{0}\end{cases}
$$

where $x_{1}^{+}=I\left(x_{1}\right)$.

Let us denote $x$ by $x_{0}^{+}$. Since $s_{0}<\infty$, the process continues from $x_{1}^{+}$onwards. If $\phi\left(x_{1}^{+}\right)=\infty$, then we define

$$
\tilde{\pi}_{x}(t)=\pi\left(x_{1}^{+}, t-s_{0}\right) \quad \text { for all } t \geqslant s_{0} .
$$

But, if $\phi\left(x_{1}^{+}\right)=s_{1}<\infty$ then $\pi\left(x_{1}^{+}, s_{1}\right)=x_{2} \in M$ and $\pi\left(x_{1}^{+}, t-s_{0}\right) \notin M$ for $s_{0}<t<s_{0}+s_{1}$. Thus, we define $\tilde{\pi}_{x}$ on $\left[s_{0}, s_{0}+s_{1}\right]$ by

$$
\tilde{\pi}_{x}(t)= \begin{cases}\pi\left(x_{1}^{+}, t-s_{0}\right), & s_{0} \leqslant t<s_{0}+s_{1} \\ x_{2}^{+}, & t=s_{0}+s_{1}\end{cases}
$$

where $x_{2}^{+}=I\left(x_{2}\right)$. Now, the process continues from $x_{2}^{+}$onwards.

The previous process ends after a finite number of steps provided $\phi\left(x_{n}^{+}\right)=\infty$ for some $n \in \mathbb{N}_{0}$. However, this process continues infinitely if $\phi\left(x_{n}^{+}\right)<\infty$ for all $n \in \mathbb{N}_{0}$. In this last case, $\tilde{\pi}_{x}$ 
is defined on the interval $[0, T(x))$, where

$$
T(x)=\sum_{i=0}^{\infty} s_{i} .
$$

Remark 2.2.1. Sometimes we will use the term impulsive semiflow instead of impulsive semidynamical system.

Definition 2.2.6. The positive impulsive trajectory of a point $x \in X$ is defined by the set $\tilde{\pi}^{+}(x)=$ $\{\tilde{\pi}(x, t): t \in[0, T(x))\}$. Given a subset $A \subset X$, we set

$$
\tilde{\pi}^{+}(A)=\bigcup_{x \in A} \tilde{\pi}^{+}(x)
$$

Analogously to the non-impulsive case, an impulsive semidynamical system satisfies the following standard properties stated in Lemma 2.2.1.

Lemma 2.2.1. (BONOTTO, 2007, Proposition 2.1) Let $(X, \pi, M, I)$ be an ISS. Then:

(a) $\tilde{\pi}(x, 0)=x$ for all $x \in X$

(b) $\tilde{\pi}(\tilde{\pi}(x, t), s)=\tilde{\pi}(x, t+s)$ for all $x \in X$ and for all $t, s \in[0, T(x))$ such that $t+s \in[0, T(x))$.

Throughout this work, we shall assume the following condition:

$$
T(x)=\infty \quad \text { for all } \quad x \in X
$$

Remark 2.2.2. Let $x \in X$ be given. If $\phi\left(x_{j}^{+}\right)<\infty$ for every $j \in \mathbb{N}_{0}$ and $t \geqslant \phi(x)$, then there exist $k \in \mathbb{N}$ and $t^{\prime} \in\left[0, \phi\left(x_{k}^{+}\right)\right)$such that

$$
t=\sum_{j=0}^{k-1} \phi\left(x_{j}^{+}\right)+t^{\prime} .
$$

Consequently,

$$
\tilde{\pi}(x, t)=\pi\left(x_{k}^{+}, t^{\prime}\right) .
$$

Definition 2.2.7. A time $t \in \mathbb{R}_{+}$is called a jump time of $x \in X$ if there exists $k \in \mathbb{N}$ such that $t=\sum_{j=0}^{k-1} \phi\left(x_{j}^{+}\right)$. Otherwise, $t$ is said to be a non-jump time.

In what follows, we exhibit the concepts of positive invariance, stationary points and periodic points in the context of impulsive semidynamical systems. 
Definition 2.2.8. A set $A \subset X$ is called positively $\tilde{\pi}$-invariant if $\tilde{\pi}^{+}(A) \subset A$.

Definition 2.2.9. Let $(X, \pi, M, I)$ be an ISS.

(i) A point $x \in X$ is said to be a stationary point if $\tilde{\pi}(x, t)=x$ for all $t \geqslant 0$.

(ii) A point $x \in X$ is said to be a periodic point if $\tilde{\pi}(x, t)=x$ for some $t>0$ and $x$ is not stationary. A positive trajectory $\tilde{\pi}^{+}(x), x \in X$, is called periodic if each point $y \in \tilde{\pi}^{+}(x)$ is periodic.

Definition 2.2.10 concerns the concepts of "limit sets" in impulsive semidynamical systems.

Definition 2.2.10. Let $(X, \pi, M, I)$ be an ISS and $A \subset X$.

(i) The impulsive positive limit set of $A$ is given by

$$
\begin{gathered}
\tilde{\omega}^{+}(A)=\left\{y \in X: \text { there are sequences }\left\{x_{n}\right\}_{n \in \mathbb{N}} \subset A \text { and }\left\{t_{n}\right\}_{n \in \mathbb{N}} \subset \mathbb{R}_{+}\right. \\
\text {such that } \left.t_{n} \stackrel{n \rightarrow \infty}{\longrightarrow \infty} \text { and } \tilde{\pi}\left(x_{n}, t_{n}\right) \stackrel{n \rightarrow \infty}{\longrightarrow} y\right\}
\end{gathered}
$$

(ii) The impulsive positive prolongational limit set of $A$ is given by

$$
\begin{gathered}
\tilde{J}^{+}(A)=\left\{y \in X: \text { there are sequences }\left\{x_{n}\right\}_{n \in \mathbb{N}} \subset X \text { and }\left\{t_{n}\right\}_{n \in \mathbb{N}} \subset \mathbb{R}_{+}\right. \\
\text {such that } \left.d\left(x_{n}, A\right) \stackrel{n \rightarrow \infty}{\longrightarrow} 0, t_{n} \stackrel{n \rightarrow \infty}{\longrightarrow} \infty \text { and } \tilde{\pi}\left(x_{n}, t_{n}\right) \stackrel{n \rightarrow \infty}{\longrightarrow} y\right\} .
\end{gathered}
$$

For each $x \in X$, we set $\tilde{\omega}^{+}(x)=\tilde{\omega}^{+}(\{x\})$ and $\tilde{J}^{+}(x)=\tilde{J}^{+}(\{x\})$. Note that $\tilde{\omega}^{+}(A) \subset \tilde{J}^{+}(A)$ for every subset $A \subset X$.

The next example is a slight modification of (BONOTTO et al., 2015, Example 4.1).

Example 2.2.1. Let $X=\left\{r e^{i \theta}: r \geqslant 0,0 \leqslant \theta \leqslant \frac{\pi}{2}\right\}$ and $\pi: X \times \mathbb{R}_{+} \rightarrow X$ be the semidynamical system generated by the following system of differential equations

$$
\left\{\begin{array}{l}
\frac{d r}{d t}=-1 \\
\frac{d \theta}{d t}=0
\end{array}\right.
$$

The positive trajectories of $\left(X, \pi, \mathbb{R}_{+}\right)$are rays going to origin. Now, let

$$
M=\left\{r e^{i \theta}: r=1,0 \leqslant \theta \leqslant \frac{\pi}{2}\right\}
$$


and $I: M \rightarrow I(M)$ be given by

$$
I\left(e^{i \theta}\right)=2 e^{i \frac{2 \theta}{3}}
$$

for each $0 \leqslant \theta \leqslant \frac{\pi}{2}$.

Note that if $z=R e^{i \gamma}$ with $R>1$ and $0<\gamma<\frac{\pi}{2}$ then

$$
\tilde{\omega}^{+}(z)=\tilde{J}^{+}(z)=\left\{r e^{i \theta}: 1 \leqslant r \leqslant 2 \text { and } \theta=0\right\} .
$$

The set $A=\left\{r e^{i \theta}: 1<r \leqslant 2\right.$ and $\left.0 \leqslant \theta \leqslant \frac{\pi}{2}\right\}$ is positively $\tilde{\pi}$-invariant. See Figure 2.4.

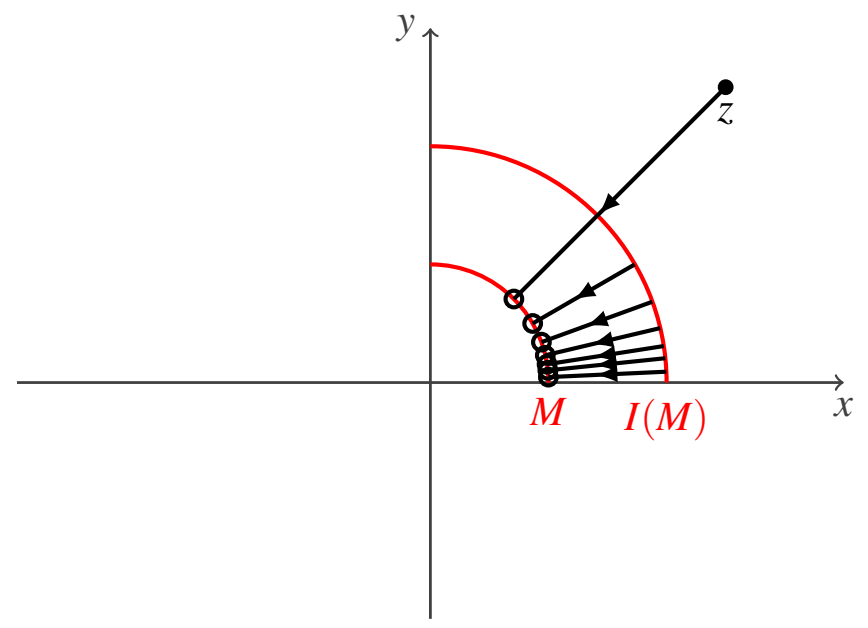

Figure 2.4: Positive impulsive trajectory of a point $z=R e^{i \gamma}$ with $R>2$ and $0<\gamma<\frac{\pi}{2}$.

Definition 2.2.11 gives us the concept of a region of weak attraction for impulsive systems.

Definition 2.2.11. Let $(X, \pi, M, I)$ be an ISS. The impulsive region of weak attraction of a set $A \subset X$ is given by

$$
\begin{aligned}
\tilde{P}_{w}^{+}(A)= & \left\{y \in X: \text { for every neighborhood } U \text { of } A \text {, there is a sequence }\left\{t_{n}\right\}_{n \in \mathbb{N}} \subset \mathbb{R}_{+},\right. \\
& \left.t_{n} \stackrel{n \rightarrow \infty}{\longrightarrow} \infty, \text { such that } \tilde{\pi}\left(y, t_{n}\right) \in U \text { for every } n \in \mathbb{N}\right\} .
\end{aligned}
$$

By (BONOTTO; FEDERSON, 2007, Lemma 3.1), an impulsive region of weak attraction is positively $\tilde{\pi}$-invariant.

Next, we present the versions of the stabilities presented in Definition 2.1.9 in the impulsive case. 
Definition 2.2.12. Let $(X, \pi, M, I)$ be an ISS. A set $A \subset X$ is said to be:

(i) orbitally $\tilde{\pi}$-stable, if for every neighborhood $U$ of $A$, there is a positively $\tilde{\pi}$-invariant neighborhood $V$ of $A$ such that $V \subset U$;

(ii) uniformly $\tilde{\pi}$-stable, if for every $\varepsilon>0$ there is $\delta=\delta(\varepsilon)>0$ such that

$$
\tilde{\pi}(B(A, \delta),[0, \infty)) \subset B(A, \varepsilon)
$$

(iii) weak $\tilde{\pi}$-stable, if $\tilde{P}_{w}^{+}(A)$ is a neighborhood of $A$;

(iv) asymptotically $\tilde{\pi}$-stable, if $A$ is both weak $\tilde{\pi}$-attractor and orbitally $\tilde{\pi}$-stable.

Example 2.2.2. In Example 2.2.1, let $A=\left\{r e^{i \theta}: 1 \leqslant r \leqslant 2\right.$ and $\left.0 \leqslant \theta \leqslant \frac{\pi}{2}\right\}$. Note that $\tilde{P}_{w}^{+}(A)=$ $\left\{r e^{i \theta}: r>1\right.$ and $\left.0 \leqslant \theta \leqslant \frac{\pi}{2}\right\}$. Thus, $A$ is not weak $\tilde{\pi}$-stable. Now, let $B=\{0\} \cup A$. Here, $\tilde{P}_{w}^{+}(B)=\mathbb{R}^{2}$. Note that $B$ is not asymptotically $\tilde{\pi}$-stable but $\{0\}$ is asymptotically $\tilde{\pi}$-stable.

Next, we exhibit an auxiliary result.

Theorem 2.2.1. (BONOTTO; GRULHA JR, 2010, Theorem 3.4) Let $(X, \pi, M, I)$ be an ISS. Assume that $X$ is locally compact and $A$ is a compact subset of $X$. Then $A$ is orbitally $\tilde{\pi}$-stable if and only if $A$ is uniformly $\tilde{\pi}$-stable.

\subsubsection{Continuity of the impact function $\phi$}

In this subsection, we discuss the continuity of the impact function $\phi$ presented in Definition 2.2.4. The continuity of $\phi$ plays an important role to assure the well behavior of the trajectories of impulsive semidynamical systems. The results presented in this subsection were established by Ciesielsky in (CIESIELSKI, 2004a).

Example 2.2.3. Let $\left(\mathbb{R}^{2}, \pi, M, I\right)$ be an ISS, where $M=\{(0, y): y \leqslant 0\}, I: M \rightarrow \mathbb{R}^{2}$ is given by $I(0, y)=(1, y), y \leqslant 0$, and

$$
\pi((x, y), t)=(x+t, y)
$$

for all $(x, y) \in \mathbb{R}^{2}$ and all $t \geqslant 0$. Note that $\phi(-1,0)=1$ and $\phi\left(-1, \frac{1}{n}\right)=\infty$ for all $n \in \mathbb{N}$. Thus, $\phi$ is not continuous at $(-1,0)$. More precisely, $\phi$ is not continuous on the set $\{(x, 0): x \leqslant 0\} \cup$ $\{(0, y): y \leqslant 0\}$. 
Example 2.2.4. In Example 2.2.1, $\phi$ is not continuous on the set $M$.

Ciesielsky proved in (CIESIELSKI, 2004a) that under a "strong tube condition" it is possible to obtain the continuity of $\phi$ outside of $M$. In order to present this result, we start by defining the concept of tube condition.

Definition 2.2.13. A closed subset $S$ containing $x \in X$ is called a $\lambda$-section through $x$ if there exist $\lambda>0$ and a closed subset $L$ of $X$ such that:

(i) $F(L, \lambda)=S$;

(ii) $F(L,[0,2 \lambda])$ contains a neighborhood of $x$;

(iii) $F(L, v) \cap F(L, \zeta)=\varnothing$, if $0 \leqslant v<\zeta \leqslant 2 \lambda$.

The set $F(L,[0,2 \lambda])$ is called a $\lambda$-tube and the set $L$ is called a bar. See Figure 2.5.

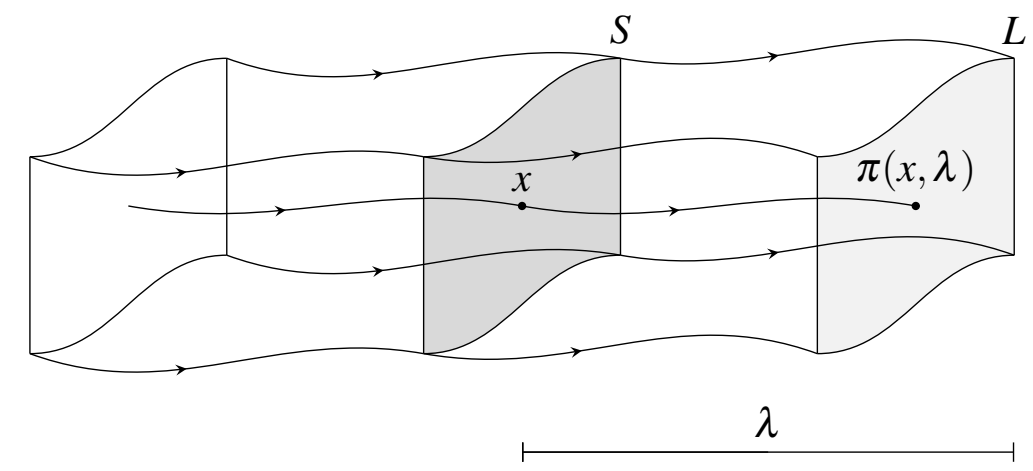

$2 \lambda$

Figure $2.5: \lambda$-tube $F(L,[0,2 \lambda])$.

Definition 2.2.14. Let $(X, \pi, M, I)$ be an ISS. We say that a point $x \in M$ satisfies the strong tube condition (or satisfies STC), if there exists a section $S$ through $x$ such that

$$
S=F(L,[0,2 \lambda]) \cap M
$$

The impulsive set $M$ satisfies STC if each point of $M$ satisfies STC.

The impulsive set presented in Example 2.2.3 does not satisfy the strong tube condition. 
Definition 2.2.15. A point $x \in X$ is called initial if $F(x, t)=\emptyset$ for all $t>0$.

The next result establishes the continuity of $\phi$ which is accomplished outside $M$.

Theorem 2.2.2. (CIESIELSKI, 2004a, Theorem 3.8) Let $(X, \pi, M, I)$ be an ISS such that each point of $M$ satisfies STC. Then $\phi$ is upper semicontinuous in $X$ and it is continuous on $X \backslash M$. Moreover, if there are no initial points in $M$ and $\phi$ is continuous at $x$ then $x \notin M$.

We can observe in Example 2.2.3 that

$$
\tilde{\pi}\left(\left(-1, \frac{1}{n}\right), 1\right) \nrightarrow \tilde{\pi}((-1,0), 1) \text { as } n \rightarrow \infty
$$

since $\tilde{\pi}\left(\left(-1, \frac{1}{n}\right), 1\right)=\pi\left(\left(-1, \frac{1}{n}\right), 1\right)=\left(0, \frac{1}{n}\right)$ and $\tilde{\pi}((-1,0), 1)=(1,0)$. However, if $M$ satisfies STC then it is possible to obtain a special convergence result for impulsive semidynamical systems. See the next result.

Lemma 2.2.2. (BONOTTO et al., 2015, Lemma 3.6, Corollary 3.9) Let $(X, \pi, M, I)$ be an ISS such that $I(M) \cap M=\varnothing$. Assume that each point of $M$ satisfies STC. Let $x \in X \backslash M$ and $\left\{x_{n}\right\}_{n \in \mathbb{N}}$ be a sequence in $X$ such that $x_{n} \stackrel{n \rightarrow \infty}{\longrightarrow} x$. Then, given $t \geqslant 0$, there exists a sequence $\left\{\eta_{n}\right\}_{n \in \mathbb{N}}$ in $\mathbb{R}_{+}$such that $\eta_{n} \stackrel{n \rightarrow \infty}{\longrightarrow} 0$ and

$$
\tilde{\pi}\left(x_{n}, t+\eta_{n}\right) \stackrel{n \rightarrow \infty}{\longrightarrow} \tilde{\pi}(x, t)
$$

Unlike semidynamical systems (without impulses), a "limit set" may not be positively invariant. For instance, in Example 2.2.1, if $p=\sqrt{2} e^{\frac{i \pi}{4}}$ then

$$
\tilde{\omega}^{+}(p)=\tilde{J}^{+}(p)=\left\{r e^{i \theta}: 1 \leqslant r \leqslant 2 \text { and } \theta=0\right\} .
$$

Thus, $\tilde{\omega}^{+}(p)$ and $\tilde{J}^{+}(p)$ are not positively $\tilde{\pi}$-invariant, since $(1,0) \in M$ belongs to these sets. However, $\tilde{\omega}^{+}(p) \backslash M$ and $\tilde{J}^{+}(p) \backslash M$ are positively $\tilde{\pi}$-invariant.

Lemma 2.2.2 is fundamental to obtain the following result.

Theorem 2.2.3. (BONOTTO; GIMENES; SOUTO, 2017, Lemma 3.37) Let $(X, \pi, M, I)$ be an ISS such that $I(M) \cap M=\varnothing$. Assume that each point of $M$ satisfies STC.

(a) If $A \subset X$ is positively $\tilde{\pi}$-invariant then $\bar{A} \backslash M$ is positively $\tilde{\pi}$-invariant.

(b) The sets $\tilde{\omega}^{+}(A) \backslash M$ and $\tilde{J}^{+}(A) \backslash M$ are positively $\tilde{\pi}$-invariant for every subset $A \subset X$. 


\subsection{Negative semisolutions}

As presented in Example 2.1.2, the absence of negative uniqueness can happens in the study of semidynamical systems. In this section, we present the introductory part of the theory of negative semisolutions in the context of semidynamical systems and in the context of impulsive semidynamical systems.

\subsubsection{Negative semisolutions for semidynamical systems}

The study of the past of a point can be done by means of negative semisolutions. The concept of a negative semisolution is stated next.

Definition 2.3.1. A negative semisolution through a point $x \in X$ is a continuous map $\sigma_{x}: I_{x} \rightarrow X$ defined on an interval $I_{x} \subset(-\infty, 0]$, with $0 \in I_{x}$, such that $\sigma_{x}(0)=x$ and $\pi\left(\sigma_{x}(t), s\right)=\sigma_{x}(t+s)$ for all $t \in I_{x}$ and $s \in \mathbb{R}_{+}$such that $t+s \in I_{x}$. If $I_{x}=(-\infty, 0]$, we say that $\sigma_{x}$ is a global negative semisolution through $x$.

Provided $X$ is a locally compact space and assuming that $\left(X, \pi, \mathbb{R}_{+}\right)$does not contain initial points, then it is possible to prolongate a negative semisolution through $x \in X$ to the interval $(-\infty, 0]$, see $(\mathrm{MCCANN}, 1977)$ for instance.

Definition 2.3.2. Let $x \in X$ and $\sigma_{x}$ be a negative semisolution through $x$ defined on $I_{x}$. The global trajectory of $x$ with respect to $\sigma_{x}$ is represented by

$$
\pi_{\sigma}(x)=\sigma_{x}\left(I_{x}\right) \cup \pi^{+}(x)
$$

The weak and strong versions of invariance are exhibited in the next definition.

Definition 2.3.3. Let $\left(X, \pi, \mathbb{R}_{+}\right)$be a semidynamical system. A set $A \subset X$ is called:

(i) negative weakly invariant, if for every $x \in A$ there is a negative semisolution $\sigma_{x}$ through $x$ such that $\sigma_{x}\left(I_{x}\right) \subset A$;

(ii) negatively strongly invariant, if for every $x \in A$ and every negative semisolution $\sigma_{x}$ through $x$ then $\sigma_{x}\left(I_{x}\right) \subset A$; 
(iii) strongly invariant, if it is positively $\pi$-invariant and negatively strongly invariant.

It is not difficult to see that the negative strong invariance implies the negative weak invariance. But the converse does not hold in general, as presented in the next example.

Example 2.3.1. In Example 2.1.3, the unit circle $S^{1}$ is negative weakly invariant but it is not negatively strongly invariant.

In Definition 2.3.4, we exhibit the negative version of limit sets.

Definition 2.3.4. Let $\left(X, \pi, \mathbb{R}_{+}\right)$be a semidynamical system and $x \in X$.

(i) The negative limit set of $x$ with respect to a global negative semisolution $\sigma_{x}$ is given by

$$
\omega_{\sigma}^{-}(x)=\left\{y \in X: \text { there exists a sequence }\left\{t_{n}\right\}_{n \in \mathbb{N}} \subset \mathbb{R}_{-}\right. \text {such that }
$$$$
\left.t_{n} \stackrel{n \rightarrow \infty}{\longrightarrow}-\infty \text { and } \sigma_{x}\left(t_{n}\right) \stackrel{n \rightarrow \infty}{\longrightarrow} y\right\}
$$

(ii) The negative prolongational limit set of $x$ is given

$$
\begin{gathered}
J^{-}(x)=\left\{y \in X: \text { there are sequences }\left\{x_{n}\right\}_{n \in \mathbb{N}} \subset X \text { and }\left\{t_{n}\right\}_{n \in \mathbb{N}} \subset \mathbb{R}_{-}\right. \text {such that } \\
x_{n} \stackrel{n \rightarrow \infty}{\longrightarrow} x, t_{n} \stackrel{n \rightarrow \infty}{\longrightarrow}-\infty \text { and for each } x_{n} \text { there exists a global negative } \\
\text { semisolution } \left.\sigma_{n} \text { through } x_{n} \text { such that } \sigma_{n}\left(t_{n}\right) \stackrel{n \rightarrow \infty}{\longrightarrow} y\right\} .
\end{gathered}
$$

For $x \in X$, the sets $\omega_{\sigma}^{-}(x)$ and $J^{-}(x)$ are closed, positively $\pi$-invariant and if $X$ is locally compact, then they are negatively weakly invariant. See (BHATIA; HAJEK, 1969; BISTROŃ, 2003; BISTROŃ, 2005) for more details.

Lemma 2.3.1. (BHATIA; HAJEK, 1969, Proposition 8.6.2) Let $A \subset X$ and $X$ be locally compact. The region of weak attraction $P_{w}^{+}(A)$ is strongly invariant.

\subsubsection{Negative semisolutions for impulsive semidynamical systems}

In this subsection, we investigate the backward behavior of impulsive semidynamical systems. The first study of impulsive negative semisolutions was introduced in (AFONSO; BONOTTO; JIMENEZ, 2015).

In order to define the concept of impulsive negative semisolutions with respect to an ISS $(X, \pi, M, I)$, we shall assume throughout this subsection the following general conditions: 
H1) Any negative semisolution through $x \in X$ is defined on $I_{x}=(-\infty, 0]$.

H2) The impulsive function $I: M \rightarrow N$ of the ISS $(X, \pi, M, I)$ is a homeomorphism with inverse $J: N \rightarrow M$, where $N=I(M)$.

H3) $I(M) \cap M=\emptyset$.

Remark 2.3.1. Since we are assuming that $I(M) \cap M=\emptyset$, if $x \in M$ then there are no $y \in X$ and $t>0$ such that $\tilde{\pi}(y, t)=x$, i.e., $x$ is an initial point in $(X, \pi, M, I)$. Hence, we cannot define an impulsive negative semisolution through $x \in M$ in $(X, \pi, M, I)$.

In the next lines, we construct an impulsive negative semisolution through a point $x \in X \backslash M$. For that, let $\sigma_{x}:(-\infty, 0] \rightarrow X$ be a global negative semisolution through $x \in X \backslash M$ w.r.t. $\left(X, \pi, \mathbb{R}_{+}\right)$. We have some cases to consider:

Case 1: $x \notin N$.

Subcase 1.1: There is $s<0$ such that $\sigma_{x}(s) \in M, \sigma_{x}((s, 0]) \cap M=\emptyset$ and $\sigma_{x}([s, 0]) \cap N=\emptyset$.

Since $\sigma_{x}(s)$ is an initial point in $(X, \pi, M, I)$, we define $\tilde{\sigma}_{x}$ on $[s, 0]$ by

$$
\tilde{\sigma}_{x}(t)=\sigma_{x}(t)
$$

and $I_{x}=[s, 0]$ is the maximal interval of existence of $\tilde{\sigma}_{x}$.

Subcase 1.2: $\sigma_{x}(t) \notin M \cup N$ for all $t \leqslant 0$.

In this case, we define $\tilde{\sigma}_{x}$ on $I_{x}=(-\infty, 0]$ by $\tilde{\sigma}_{x}(t)=\sigma_{x}(t)$.

Subcase 1.3: there is $r_{0}<0$ such that $\sigma_{x}\left(r_{0}\right)=a_{1} \in N$ and $\sigma_{x}(t) \notin N \cup M$ for $r_{0}<t \leqslant 0$.

Here, we define $\tilde{\sigma}_{x}$ on $\left[r_{0}, 0\right]$ by $\tilde{\sigma}_{x}(t)=\sigma_{x}(t)$. Let $a_{1}^{-}=J\left(a_{1}\right) \in M$ and $\sigma_{a_{1}^{-}}$be an arbitrary global negative semisolution through $a_{1}^{-}$.

- If $\sigma_{a_{1}^{-}}(t) \notin M \cup N$ for all $t<0$, then we define $\tilde{\sigma}_{x}(t)=\sigma_{a_{1}^{-}}\left(t-r_{0}\right)$ for $-\infty<t<r_{0}$. In this way, $\tilde{\sigma}_{x}$ is defined on $I_{x}=(-\infty, 0]$ by

$$
\tilde{\sigma}_{x}(t)= \begin{cases}\sigma_{x}(t), & r_{0} \leqslant t \leqslant 0, \\ \sigma_{a_{1}^{-}}\left(t-r_{0}\right), & -\infty<t<r_{0} .\end{cases}
$$


- If there exists $r_{1}<0$ such that $\sigma_{a_{1}^{-}}\left(r_{1}\right) \in M, \sigma_{a_{1}^{-}}\left(\left(r_{1}, 0\right)\right) \cap M=\emptyset$ and $\sigma_{a_{1}^{-}}\left(\left[r_{1}, 0\right]\right) \cap N=\emptyset$, then we define $\tilde{\sigma}_{x}(t)=\sigma_{a_{1}^{-}}\left(t-r_{0}\right)$ for $r_{0}+r_{1} \leqslant t<r_{0}$. Thus, $\tilde{\sigma}_{x}$ is defined on $I_{x}=\left[r_{0}+r_{1}, 0\right]$ by

$$
\tilde{\sigma}_{x}(t)= \begin{cases}\sigma_{x}(t), & r_{0} \leqslant t \leqslant 0, \\ \sigma_{a_{1}^{-}}\left(t-r_{0}\right), & r_{0}+r_{1} \leqslant t<r_{0} .\end{cases}
$$

- If there exists $r_{1}<0$ such that $\sigma_{a_{1}^{-}}\left(r_{1}\right)=a_{2} \in N$ and $\sigma_{a_{1}^{-}}(t) \notin N \cup M$ for $r_{1}<t<0$, then $\tilde{\sigma}_{x}$ is defined on $\left[r_{0}+r_{1}, 0\right]$ by

$$
\tilde{\sigma}_{x}(t)= \begin{cases}\sigma_{x}(t), & r_{0} \leqslant t \leqslant 0, \\ \sigma_{a_{1}^{-}}\left(t-r_{0}\right), & r_{0}+r_{1} \leqslant t<r_{0} .\end{cases}
$$

Now, we set $a_{2}^{-}=J\left(a_{2}\right)$ and the previous process continues from $a_{2}^{-}$and so on.

Case 2: $x \in N$.

Denote $a_{1}=x$ and $a_{1}^{-}=J(x) \in M$. Let $\sigma_{a_{1}^{-}}$be an arbitrary global negative semisolution through $a_{1}^{-}$.

Subcase 2.1: $\sigma_{a_{1}^{-}}(t) \notin M \cup N$ for all $t<0$.

In this case, we define $\tilde{\sigma}_{x}$ on $I_{x}=(-\infty, 0]$ by

$$
\tilde{\sigma}_{x}(t)= \begin{cases}x, & t=0, \\ \sigma_{a_{1}^{-}}(t), & -\infty<t<0 .\end{cases}
$$

Subcase 2.2: There exists $r_{0}<0$ such that $\sigma_{a_{1}^{-}}\left(r_{0}\right) \in M, \sigma_{a_{1}^{-}}\left(\left(r_{0}, 0\right)\right) \cap M=\emptyset$ and $\sigma_{a_{1}^{-}}\left(\left[r_{0}, 0\right]\right) \cap$ $N=\emptyset$.

Here, $\tilde{\sigma}_{x}$ is defined on $I_{x}=\left[r_{0}, 0\right]$ by

$$
\tilde{\sigma}_{x}(t)= \begin{cases}x, & t=0 \\ \sigma_{a_{1}^{-}}(t), & r_{0} \leqslant t<0 .\end{cases}
$$

Subcase 2.3: There exists $r_{0}<0$ such that $\sigma_{a_{1}^{-}}\left(r_{0}\right)=a_{2} \in N$ and $\sigma_{a_{1}^{-}}(t) \notin M \cup N$ for $r_{0}<t<0$.

We set $\tilde{\sigma}_{x}$ on $\left[r_{0}, 0\right]$ by

$$
\tilde{\sigma}_{x}(t)= \begin{cases}x, & t=0 \\ \sigma_{a_{1}^{-}}(t), & r_{0} \leqslant t<0\end{cases}
$$


and $a_{2}^{-}=J\left(a_{2}\right)$. Let $\sigma_{a_{2}^{-}}$be an arbitrary global negative semisolution through $a_{2}^{-}$.

- If $\sigma_{a_{2}^{-}}(t) \notin M \cup N$ for all $t<0$, then $\tilde{\sigma}_{x}$ is defined on $I_{x}=(-\infty, 0]$ by

$$
\tilde{\sigma}_{x}(t)= \begin{cases}x, & t=0, \\ \sigma_{a_{1}^{-}}(t), & r_{0} \leqslant t<0 \\ \sigma_{a_{2}^{-}}\left(t-r_{0}\right), & -\infty<t<r_{0}\end{cases}
$$

- If there exists $r_{1}<0$ such that $\sigma_{a_{2}^{-}}\left(r_{1}\right) \in M, \sigma_{a_{2}^{-}}\left(\left(r_{1}, 0\right)\right) \cap M=\emptyset$ and $\sigma_{a_{2}^{-}}\left(\left[r_{1}, 0\right]\right) \cap N=\emptyset$, then $\tilde{\sigma}_{x}$ is defined on $I_{x}=\left[r_{0}+r_{1}, 0\right]$ by

$$
\tilde{\sigma}_{x}(t)= \begin{cases}x, & t=0 \\ \sigma_{a_{1}^{-}}(t), & r_{0} \leqslant t<0 \\ \sigma_{a_{2}^{-}}\left(t-r_{0}\right), & r_{0}+r_{1} \leqslant t<r_{0}\end{cases}
$$

- If there exists $r_{1}<0$ such that $\sigma_{a_{2}^{-}}\left(r_{1}\right)=a_{3} \in N$ and $\sigma_{a_{2}^{-}}(t) \notin N \cup M$ for $r_{0}+r_{1}<t<r_{0}$, then we define $\tilde{\sigma}_{x}$ on $\left[r_{0}+r_{1}, 0\right]$ by

$$
\tilde{\sigma}_{x}(t)= \begin{cases}x, & t=0 \\ \sigma_{a_{1}^{-}}(t), & r_{0} \leqslant t<0 \\ \sigma_{a_{2}^{-}}\left(t-r_{0}\right), & r_{0}+r_{1} \leqslant t<r_{0}\end{cases}
$$

we set $a_{3}=J\left(a_{3}\right)$ and the process continues from $a_{3}^{-}$and so on.

As in the non-impulsive case, we also have the following property.

Proposition 2.3.1. (AFONSO; BONOTTO; JIMENEZ, 2015, Lemma 4.1) Let $x \in X \backslash M$ and $\tilde{\sigma}_{x}$ be an impulsive negative semisolution through $x$ defined on $I_{x}$. Then $\tilde{\sigma}_{x}(0)=x$ and $\tilde{\pi}\left(\tilde{\sigma}_{x}(t), s\right)=$ $\tilde{\sigma}_{x}(t+s)$ for all $t \in I_{x}$ and $s \geqslant 0$, such that $t+s \in I_{x}$.

Next, we present the concepts of global trajectory and invariance.

Definition 2.3.5. Let $x \in X \backslash M$ and $\tilde{\sigma}_{x}$ be an impulsive negative semisolution through $x$ defined on $I_{x}$. The impulsive global trajectory of $x$ with respect to $\tilde{\sigma}_{x}$ is represented by

$$
\tilde{\pi}_{\sigma}(x)=\tilde{\sigma}_{x}\left(I_{x}\right) \cup \tilde{\pi}^{+}(x) .
$$


Definition 2.3.6. A nonempty subset $A$ of $X$ is said to be:

(i) negatively strongly invariant, if $\tilde{\sigma}_{x}\left(I_{x}\right) \subset A$ for any $x \in A \backslash M$ and for any impulsive negative semisolution $\tilde{\sigma}_{x}$ through $x$, where $I_{x}$ is the maximal interval of existence of $\tilde{\sigma}_{x}$;

(ii) negatively weakly invariant, if for every $x \in A \backslash M$ there exists an impulsive negative semisolution $\tilde{\sigma}_{x}$ through $x$ such that $\tilde{\sigma}_{x}\left(I_{x}\right) \subset A$, where $I_{x}$ is the maximal interval of existence of $\tilde{\sigma}_{x}$

(iii) strongly (weakly) invariant, if it is positively $\tilde{\pi}$-invariant and negatively strongly (weakly) invariant.

According to (AFONSO; BONOTTO; JIMENEZ, 2015, Lemma 4.2), the set $\tilde{\sigma}_{x}\left(I_{x}\right), x \in$ $X \backslash M$, is negatively weakly invariant for any impulsive negative semisolution $\tilde{\sigma}_{x}$ through $x$. Thus, $\tilde{\pi}_{\sigma}(x)$ is weakly invariant.

We end this subsection with a result concerning the negative invariance of an impulsive region of weak attraction.

Lemma 2.3.2. Let $A \subset X$. The impulsive region of weak attraction $\tilde{P}_{w}^{+}(A)$ is negatively strongly invariant.

Proof. Let $x \in \tilde{P}_{w}^{+}(A) \backslash M, \tilde{\sigma}_{x}$ be an impulsive negative semisolution through $x$ defined on the maximal interval of existence $I_{x}$, and $t \in I_{x}$. Given an arbitrary neighborhood $U$ of $A$, there exists a sequence $\left\{t_{n}\right\}_{n \in \mathbb{N}} \subset \mathbb{R}_{+}$such that $t_{n} \stackrel{n \rightarrow \infty}{\longrightarrow} \infty$ and $\tilde{\pi}\left(x, t_{n}\right) \in U$ for every $n \in \mathbb{N}$. Let $n_{0} \in \mathbb{N}$ be such that $t_{n}>t$ for all $n \geqslant n_{0}$. Now, note that

$$
\tilde{\pi}\left(\tilde{\sigma}_{x}(t), t_{n}-t\right)=\tilde{\pi}\left(\tilde{\pi}\left(\tilde{\sigma}_{x}(t),-t\right), t_{n}\right)=\tilde{\pi}\left(x, t_{n}\right) \in U
$$

for all $n \geqslant n_{0}$. Thus, $\tilde{\sigma}_{x}(t) \in \tilde{P}_{w}^{+}(A)$ and the result is complete.

The reader may consult (AFONSO; BONOTTO; JIMENEZ, 2015) to obtain more details about the properties of impulsive negative semisolutions. 


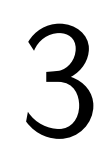

\section{PARALLELIZABLE SEMIDYNAMICAL \\ SYSTEMS}

In this chapter, we introduce the notion of parallelizability in the context of semidynamical systems. The results of this chapter are presented in the paper (BONOTTO; PACIFICO, 2021b).

\subsection{Parallelizable semidynamical systems}

As presented in (BHATIA; SZEGÖ, 2002; ANTOSIEWICZ; DUGUNDJI, 1960; HáJEK, $1971)$, a dynamical system $(X, \pi, \mathbb{R})$ is called parallelizable if there exist a subset $S \subset X$ and a homeomorphism $h: X \rightarrow S \times \mathbb{R}$ such that

- $\pi(S, \mathbb{R})=X$, and

- $h(\pi(x, t))=(x, t)$ for every $x \in S$ and $t \in \mathbb{R}$.

However, as presented in Section 2.1, Chapter 2, the "past trajectory" of a given point $x$ may be not unique. In this way, it is necessary a suitable notion of parallelizability in the context of semidynamical systems.

In what follows, we establish the theory of parallelizability in semidynamical systems.

Definition 3.1.1. A subset $S \subset X$ is called a base for the system $\left(X, \pi, \mathbb{R}_{+}\right)$if

$$
X=F\left(S, \mathbb{R}_{+}\right) \bigcup \pi\left(S, \mathbb{R}_{+}\right)
$$


Definition 3.1.2. A semidynamical system $\left(X, \pi, \mathbb{R}_{+}\right)$is said to be parallelizable, if there exist a base $S \subset X$ and a continuous function $h: X \rightarrow S \times \mathbb{R}_{+}$satisfying the following conditions:

(i) $h(\pi(x, t))=(x, t)$ for all $x \in S$ and $t \in \mathbb{R}_{+}$;

(ii) $h(F(x, t))=(x, t)$ for all $x \in S$ and $t \in \mathbb{R}_{+}$.

Example 3.1.1. Let $\left(X, \pi, \mathbb{R}_{+}\right)$be the semidynamical system presented in Example 2.1.2, whose trajectories are presented in Figure 3.1.

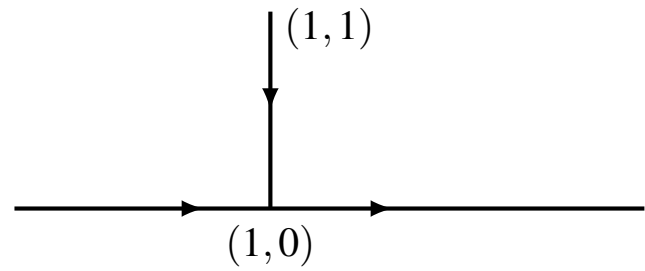

Figure 3.1: Positive trajectories of the system $\left(X, \pi, \mathbb{R}_{+}\right)$.

The set $S_{x}=\{(x, 0)\}$ is a base for $\left(X, \pi, \mathbb{R}_{+}\right)$only if $x \geqslant 1$. Fix $x_{0} \geqslant 1$. Set $S_{x_{0}}=\left\{\left(x_{0}, 0\right)\right\}$ and define $h: X \rightarrow S_{x_{0}} \times \mathbb{R}_{+}$by $h(x, y)=\left(\left(x_{0}, 0\right),\left|x-y-x_{0}\right|\right)$, for all $(x, y) \in X$. Thus, we obtain $X=F\left(S_{x_{0}}, \mathbb{R}_{+}\right) \cup \pi\left(S_{x_{0}}, \mathbb{R}_{+}\right), h$ is continuous on $X$ and

$$
h\left(\pi\left(\left(x_{0}, 0\right), t\right)\right)=h\left(x_{0}+t, 0\right)=\left(\left(x_{0}, 0\right),|t|\right)=\left(\left(x_{0}, 0\right), t\right), \quad \text { for all } \quad t \in \mathbb{R}_{+} .
$$

Since

$$
F\left(\left(x_{0}, 0\right), \lambda\right)= \begin{cases}\left\{\left(x_{0}-\lambda, 0\right)\right\} & \text { if } 0 \leqslant \lambda \leqslant x_{0}-1, \\ \left\{\left(x_{0}-\lambda, 0\right),\left(1, \lambda-x_{0}+1\right)\right\} & \text { if } x_{0}-1<\lambda \leqslant x_{0}, \\ \left\{\left(x_{0}-\lambda, 0\right)\right\} & \text { if } \lambda>x_{0},\end{cases}
$$

we have $h\left(F\left(\left(x_{0}, 0\right), t\right)\right)=\left(\left(x_{0}, 0\right), t\right)$ for all $t \in \mathbb{R}_{+}$. Hence, $\left(X, \pi, \mathbb{R}_{+}\right)$is parallelizable.

In Lemma 3.1.1, we prove that there exists a unique function $h$ associated with a base $S$.

Lemma 3.1.1. If $\left(X, \pi, \mathbb{R}_{+}\right)$is parallelizable, then for each base $S$ there exists a unique function $h: X \rightarrow S \times \mathbb{R}_{+}$satisfying conditions $(i)$ and (ii) of Definition 3.1.2.

Proof. Let $S$ be a base for $\left(X, \pi, \mathbb{R}_{+}\right)$and $h_{1}, h_{2}: X \rightarrow S \times \mathbb{R}_{+}$be functions satisfying conditions (i) and (ii) of Definition 3.1.2. 
Let $x \in X$ be arbitrary. Since $X=F\left(S, \mathbb{R}_{+}\right) \cup \pi\left(S, \mathbb{R}_{+}\right)$, let us assume initially that $x \in$ $\pi\left(S, \mathbb{R}_{+}\right)$. Thus, there are $y \in S$ and $t \in \mathbb{R}_{+}$such that $x=\pi(y, t)$, consequently, using condition $(i)$, we have

$$
h_{1}(x)=h_{1}(\pi(y, t))=(y, t)=h_{2}(\pi(y, t))=h_{2}(x) .
$$

On the other side, if $x \in F\left(S, \mathbb{R}_{+}\right)$then there are $y \in S$ and $t \in \mathbb{R}_{+}$such that $x \in F(y, t)$. Thus, using condition (ii),

$$
h_{1}(x)=(y, t)=h_{2}(x)
$$

Hence, $h_{1}(x)=h_{2}(x)$ for all $x \in X$.

In Lemma 3.1.2, we exhibit some topological properties for a base $S$ of a parallelizable semidynamical system.

Lemma 3.1.2. Let $\left(X, \pi, \mathbb{R}_{+}\right)$be parallelizable with base $S$. The following conditions hold:

(a) $S$ is closed in $X$;

(b) $S, \pi(S,(0, \infty))$ and $F(S,(0, \infty))$ are pairwise disjoint sets;

(c) $\pi\left(S, \mathbb{R}_{+}\right)$and $F\left(S, \mathbb{R}_{+}\right)$are closed sets in $X$;

(d) $\pi(S,(0, \infty))$ and $F(S,(0, \infty))$ are open sets in $X$.

Proof. Since $\left(X, \pi, \mathbb{R}_{+}\right)$is parallelizable, one can find a continuous function $h: X \rightarrow S \times \mathbb{R}_{+}$ satisfying conditions $(i)$ and $(i i)$ of Definition 3.1.2.

(a) The set $S$ is closed in $X$ as $h^{-1}(S \times\{0\})=S$ and $S \times\{0\}$ is closed in $S \times \mathbb{R}_{+}$.

(b) Let us show that the sets $\pi(S,(0, \infty))$ and $F(S,(0, \infty))$ are pairwise disjoint. Suppose there is $y \in F(S,(0, \infty)) \bigcap \pi(S,(0, \infty))$. Then there exist $z_{1}, z_{2} \in S$ and $t_{1}, t_{2} \in(0, \infty)$ with $\pi\left(z_{1}, t_{1}\right)=y$ and $\pi\left(y, t_{2}\right)=z_{2}$. Consequently,

$$
\left(z_{1}, t_{1}+t_{2}\right) \stackrel{(i)}{=} h\left(\pi\left(z_{1}, t_{1}+t_{2}\right)\right)=h\left(\pi\left(y, t_{2}\right)\right)=h\left(z_{2}\right) \stackrel{(i)}{=}\left(z_{2}, 0\right),
$$

which is a contradiction as $t_{1}, t_{2}>0$. Thus, $F(S,(0, \infty)) \bigcap \pi(S,(0, \infty))=\emptyset$.

Let us show that the sets $\pi(S,(0, \infty))$ and $S$ are pairwise disjoint. Suppose there is $y \in$ $\pi(S,(0, \infty)) \bigcap S$. In this case, there exist $w \in S$ and $s \in(0, \infty)$ such that $\pi(w, s)=y$. Thus,

$$
(w, s) \stackrel{(i)}{=} h(\pi(w, s))=h(y)=h(\pi(y, 0)) \stackrel{(i)}{=}(y, 0),
$$


which is a contradiction as $s>0$. Hence, $\pi(S,(0, \infty)) \bigcap S=\emptyset$.

Now, we show that $F(S,(0, \infty))$ and $S$ are pairwise disjoint. As before, suppose there is $y \in F(S,(0, \infty)) \bigcap S$. In this case, there exist $v \in S$ and $r \in(0, \infty)$ such that $\pi(y, r)=v$. Then

$$
(y, r) \stackrel{(i)}{=} h(\pi(y, r))=h(v)=h(\pi(v, 0)) \stackrel{(i)}{=}(v, 0)
$$

which leads us again to a contradiction. Hence, $F(S,(0, \infty)) \bigcap S=\emptyset$.

(c) Let $\left\{x_{n}\right\}_{n \in \mathbb{N}} \subset F\left(S, \mathbb{R}_{+}\right)$be a sequence such that $x_{n} \stackrel{n \rightarrow \infty}{\longrightarrow} x$. By continuity of $h, h\left(x_{n}\right) \stackrel{n \rightarrow \infty}{\longrightarrow}$ $h(x)$. Moreover, there is $(z, t) \in S \times \mathbb{R}_{+}$such that $h(x)=(z, t)$ as the range of $h$ is in $S \times \mathbb{R}_{+}$. On the other hand, for each $n \in \mathbb{N}$, there exist $z_{n} \in S$ and $t_{n} \in \mathbb{R}_{+}$such that $\pi\left(x_{n}, t_{n}\right)=z_{n}$. Using the properties of $h$ we obtain

$$
\left(z_{n}, t_{n}\right)=h\left(x_{n}\right) \stackrel{n \rightarrow \infty}{\longrightarrow} h(x)=(z, t) .
$$

Consequently, $\pi(x, t)=z$ as $z_{n} \stackrel{n \rightarrow \infty}{\longrightarrow} z$ and $\pi\left(x_{n}, t_{n}\right) \stackrel{n \rightarrow \infty}{\longrightarrow} \pi(x, t)$. Thus, $x \in F\left(S, \mathbb{R}_{+}\right)$and $F\left(S, \mathbb{R}_{+}\right)$ is closed in $X$.

Now, let $\left\{z_{n}\right\}_{n \in \mathbb{N}} \subset \pi\left(S, \mathbb{R}_{+}\right)$be a sequence such that $z_{n} \stackrel{n \rightarrow \infty}{\longrightarrow} z$. By continuity of $h, h\left(z_{n}\right) \stackrel{n \rightarrow \infty}{\longrightarrow}$ $h(z)$. Let $(w, r) \in S \times \mathbb{R}_{+}$be such that $h(z)=(w, r)$. Also, for each $n \in \mathbb{N}$, there exist $w_{n} \in S$ and $r_{n} \in \mathbb{R}_{+}$such that $\pi\left(w_{n}, r_{n}\right)=z_{n}$. Using the properties of $h$ we obtain

$$
\left(w_{n}, r_{n}\right)=h\left(z_{n}\right) \stackrel{n \rightarrow \infty}{\longrightarrow} h(z)=(w, r) .
$$

Thus, $w_{n} \stackrel{n \rightarrow \infty}{\longrightarrow} w$ and $r_{n} \stackrel{n \rightarrow \infty}{\longrightarrow} r$. As $\pi\left(w_{n}, r_{n}\right)=z_{n}, n \in \mathbb{N}$, we get $z=\pi(w, r) \in \pi\left(S, \mathbb{R}_{+}\right)$. Hence, $\pi\left(S, \mathbb{R}_{+}\right)$is closed in $X$.

(d) Since $\pi(S,(0, \infty))=X \backslash F\left(S, \mathbb{R}_{+}\right)$and $F(S,(0, \infty))=X \backslash \pi\left(S, \mathbb{R}_{+}\right)$, the result follows by item $(c)$

Next, we consider a special type of base.

Definition 3.1.3. A subset $S \subset X$ is called a section for the semidynamical system $\left(X, \pi, \mathbb{R}_{+}\right)$, if for each $x \in X$ either there is a unique $t_{x} \in \mathbb{R}_{+}$such that $\pi\left(x, t_{x}\right) \in S$ or there are unique $z_{x} \in S$ and $t_{x} \in \mathbb{R}_{+}$such that $\pi\left(z_{x}, t_{x}\right)=x$.

Remark 3.1.1. Let $S \subset X$ be a section and $x \in X$. If there exists a unique $t_{x}>0$ such that $\pi\left(x, t_{x}\right) \in S$ (thus, $\left.x \notin S\right)$ then $F\left(x, \mathbb{R}_{+}\right) \cap S=\emptyset$. In the case that $x \in S$, that is, $t_{x}=0$, it follows that $F(x,(0, \infty)) \bigcap S=\emptyset$ and $\pi(S,(0, \infty)) \bigcap S=\emptyset$. Indeed, suppose there exists $w \in F\left(x, \mathbb{R}_{+}\right) \cap S$ with 
$x \notin S$. Then $\pi(w, s)=x$ for some $s \in \mathbb{R}_{+}$. But this implies that $w \in S$ and $\pi\left(w, s+t_{x}\right) \in S$. Since $S$ is a section, it contradicts the uniqueness of $t_{w}$. If $x \in S$ then the proof is analogous taking into account that $t_{x}=0$.

On the other hand, if there are unique $z_{x} \in S$ and $t_{x}>0$ such that $\pi\left(z_{x}, t_{x}\right)=x$, then $\pi\left(x, \mathbb{R}_{+}\right) \bigcap S=\emptyset$. In fact, suppose there exists $y \in S$ such that $y=\pi(x, r)$ for some $r \geqslant 0$. Let $v=\pi(y, \xi)$ with $\xi>0$. Then $\pi\left(z_{x}, t_{x}+r+\xi\right)=v=\pi(y, \xi)$ which is a contradiction.

According to Definition 3.1.3, if $\left(X, \pi, \mathbb{R}_{+}\right)$admits a section $S$, then we may define a function

$$
\begin{aligned}
\tau_{S}: X & \longrightarrow \mathbb{R}_{+} \\
x & \longmapsto \tau_{S}(x)=t_{x}
\end{aligned}
$$

namely section function. Thus, if $S$ is a section for $\left(X, \pi, \mathbb{R}_{+}\right)$then given $x \in X$,

either $\pi\left(x, \tau_{S}(x)\right) \in S \quad$ or $\quad F\left(x, \tau_{S}(x)\right)=\left\{z_{x}\right\} \subset S$.

In Example 3.1.1, the set $S_{x_{0}}=\left\{\left(x_{0}, 0\right)\right\}, x_{0} \geqslant 1$, is a section with an associated continuous section function $\tau_{S_{x_{0}}}$ given by

$$
\tau_{S_{x_{0}}}(x, y)=\left\{\begin{array}{cl}
x-x_{0} & \text { if } \quad y=0 \text { and } x>x_{0} \\
x_{0}-x & \text { if } y=0 \text { and } x \leqslant x_{0} \\
x_{0}+y-1 & \text { if } \quad x=1 \text { and } 0<y \leqslant 1
\end{array}\right.
$$

Remark 3.1.2. If $S$ is a section then the sets $S, \pi(S,(0, \infty))$ and $F(S,(0, \infty))$ are pairwise disjoint with union $X$. Further, if $S$ is a section with continuous section function $\tau_{S}$ then it is closed as $\tau_{S}(x)=0$ if and only if $x \in S$.

In what follows, we give some auxiliary results.

Lemma 3.1.3. If $S$ is a section with continuous section function $\tau_{S}$, then $\pi(S,(0, \infty))$ and $F(S,(0, \infty))$ are open in $X$.

Proof. At first, let us show that $\pi(S,(0, \infty))$ is open. For that, suppose to the contrary that there are $y \in \pi(S,(0, \infty))$ and a sequence $y_{n} \stackrel{n \rightarrow \infty}{\longrightarrow} y$ such that $y_{n} \notin \pi(S,(0, \infty)), n \in \mathbb{N}$. As $S$ is a section, 
we have $\left\{y_{n}\right\}_{n \in \mathbb{N}} \subset F(S,[0, \infty))$. Let $z_{y} \in S$ be such that $y=\pi\left(z_{y}, \tau_{S}(y)\right)$ and $w_{n} \in S$ be such that $\pi\left(y_{n}, \tau_{S}\left(y_{n}\right)\right)=w_{n}, n \in \mathbb{N}$. Since $S$ is closed and $\tau_{S}$ is continuous, we have

$$
w_{n}=\pi\left(y_{n}, \tau_{S}\left(y_{n}\right)\right) \stackrel{n \rightarrow \infty}{\longrightarrow} \pi\left(y, \tau_{S}(y)\right) \in S .
$$

Hence, $0=\tau_{S}\left(\pi\left(y, \tau_{S}(y)\right)\right)=\tau_{S}\left(\pi\left(z_{y}, 2 \tau_{S}(y)\right)\right)=2 \tau_{S}(y)$ which is an absurd as $y \notin S$. Therefore, $\pi(S,(0, \infty))$ is an open set in $X$.

Now, suppose to the contrary that there are $y \in F(S,(0, \infty))$ and a sequence $y_{n} \stackrel{n \rightarrow \infty}{\longrightarrow} y$ such that $y_{n} \notin F(S,(0, \infty)), n \in \mathbb{N}$. Thus $\left\{y_{n}\right\}_{n \in \mathbb{N}} \subset \pi(S,[0, \infty))$ since $S$ is a section. Consequently, there exists $w_{n} \in S$ such that $\pi\left(w_{n}, \tau_{S}\left(y_{n}\right)\right)=y_{n}$, for all $n \in \mathbb{N}$. Since $\tau_{S}\left(y_{n}\right) \stackrel{n \rightarrow \infty}{\longrightarrow} \tau_{S}(y)$, we get

$$
\pi\left(w_{n}, 2 \tau_{S}\left(y_{n}\right)\right)=\pi\left(y_{n}, \tau_{S}\left(y_{n}\right)\right) \stackrel{n \rightarrow \infty}{\longrightarrow} \pi\left(y, \tau_{S}(y)\right) \in S .
$$

Hence, $2 \tau_{S}\left(y_{n}\right)=\tau_{S}\left(\pi\left(w_{n}, 2 \tau_{S}\left(y_{n}\right)\right)\right) \stackrel{n \rightarrow \infty}{\longrightarrow} \tau_{S}\left(\pi\left(y, \tau_{S}(y)\right)\right)=0$, that is, $\tau_{S}(y)=0$ and $y \in S$, which is a contradiction. This shows that $F(S,(0, \infty))$ is open in $X$.

Lemma 3.1.4. Assume that $S \subset X$ is a section with continuous section function $\tau_{S}$. Let $S_{\ell}=\pi(S, \ell)$ for $\ell>0$. Then for each $\ell>0$ :

(a) the sets $F\left(S_{\ell},(\ell, \infty)\right), F\left(S_{\ell},(0, \ell]\right)$ and $\pi\left(S_{\ell}, \mathbb{R}_{+}\right)$are pairwise disjoint with union $X$;

(b) the set $S_{\ell}=\pi(S, \ell)$ is a section with continuous section function

$$
\tau_{S_{\ell}}(x)=\left\{\begin{array}{rll}
\ell+\tau_{S}(x) & \text { if } & x \in F\left(S_{\ell},(\ell, \infty)\right), \\
\ell-\tau_{S}(x) & \text { if } & x \in F\left(S_{\ell},(0, \ell]\right), \\
-\ell+\tau_{S}(x) & \text { if } & x \in \pi\left(S_{\ell}, \mathbb{R}_{+}\right)
\end{array}\right.
$$

Proof. (a) Suppose there is $y \in F\left(S_{\ell},(\ell, \infty)\right) \cap F\left(S_{\ell},(0, \ell]\right)$. Then there are $a, b \in S_{\ell}, t>\ell$ and $0<s \leqslant \ell$ such that $\pi(y, t)=a$ and $\pi(y, s)=b$. On the other hand, there are $z_{a}, z_{b} \in S$ such that $a=\pi\left(z_{a}, \ell\right)$ and $b=\pi\left(z_{b}, \ell\right)$. Thus

$$
\pi\left(z_{a}, \ell+s\right)=\pi(a, s)=\pi(y, t+s)=\pi(b, t)=\pi\left(z_{b}, \ell+t\right)
$$

which implies $\ell+s=\tau_{S}\left(\pi\left(z_{a}, \ell+s\right)\right)=\tau_{S}\left(\pi\left(z_{b}, \ell+t\right)\right)=\ell+t$. But this is a contradiction as $t \neq s$. Analogously, it is shown that $F\left(S_{\ell},(\ell, \infty)\right) \bigcap \pi\left(S_{\ell}, \mathbb{R}_{+}\right)=\emptyset$ and $F\left(S_{\ell},(0, \ell]\right) \cap \pi\left(S_{\ell}, \mathbb{R}_{+}\right)=\emptyset$.

Let us prove that $X=F\left(S_{\ell},(\ell, \infty)\right) \cup F\left(S_{\ell},(0, \ell]\right) \cup \pi\left(S_{\ell}, \mathbb{R}_{+}\right)$. Indeed, let $x \in X$. Since $X=F\left(S, \mathbb{R}_{+}\right) \cup \pi\left(S, \mathbb{R}_{+}\right)=F(S,(0, \infty)) \cup \pi\left(S, \mathbb{R}_{+}\right)$, we have two cases to consider. 
If $x \in \pi\left(S, \mathbb{R}_{+}\right)$then $x=\pi(w, t)$ for some $w \in S$ and $t \in \mathbb{R}_{+}$. If $0 \leqslant t<\ell$ then we get $x \in F\left(S_{\ell}, \ell-t\right) \subset F\left(S_{\ell},(0, \ell]\right)$. However, if $t \geqslant \ell$ then $x \in \pi\left(S_{\ell}, t-\ell\right) \subset \pi\left(S_{\ell}, \mathbb{R}_{+}\right)$.

If $x \in F(S,(0, \infty))$, then $\pi(x, s)=z$ for some $z \in S$ and $s>0$. Then $\pi(x, s+\ell)=\pi(z, \ell) \in S_{\ell}$. Hence, $x \in F\left(S_{\ell}, s+\ell\right) \subset F\left(S_{\ell},(\ell, \infty)\right)$.

Therefore, $X=F\left(S_{\ell},(\ell, \infty)\right) \cup F\left(S_{\ell},(0, \ell]\right) \cup \pi\left(S_{\ell}, \mathbb{R}_{+}\right)$.

(b) Fix $\ell>0$. Now, let $x \in X$. We are going to show that either there is a unique $t_{x} \in \mathbb{R}_{+}$ such that $\pi\left(x, t_{x}\right) \in S_{\ell}$ or there are unique $w_{x} \in S_{\ell}$ and $t_{x} \in \mathbb{R}_{+}$such that $\pi\left(w_{x}, t_{x}\right)=x$.

Case 1: $x \in F\left(S_{\ell},(\ell, \infty)\right)$.

In this case, there are $w \in S_{\ell}$ and $r>\ell$ such that $\pi(x, r)=w$. Since $w \in S_{\ell}=\pi(S, \ell)$ and $S$ is a section, there is a unique $z_{w} \in S$ such that $\pi\left(z_{w}, \ell\right)=w$ (note that $\tau_{S}(w)=\ell$ ).

We claim that $x \in F\left(S, \mathbb{R}_{+}\right)$. In fact, suppose for the sake of contradiction that there is $z_{x} \in S$ such that $\pi\left(z_{x}, \tau_{S}(x)\right)=x$. Thus

$$
\pi\left(z_{x}, \tau_{S}(x)+r\right)=\pi(x, r)=w
$$

which implies $r>\ell=\tau_{S}(w)=\tau_{S}\left(\pi\left(z_{x}, \tau_{S}(x)+r\right)\right)=\tau_{S}(x)+r \geqslant r$, a contradiction. Hence, $x \in$ $F\left(S, \mathbb{R}_{+}\right)$which means that $\pi\left(x, \tau_{S}(x)\right) \in S$. Thus,

$$
\pi\left(z_{w}, \ell+\tau_{S}(x)\right)=\pi\left(w, \tau_{S}(x)\right)=\pi\left(\pi\left(x, \tau_{S}(x)\right), r\right),
$$

which implies that

$$
\ell+\tau_{S}(x)=\tau_{S}\left(\pi\left(z_{w}, \ell+\tau_{S}(x)\right)\right)=\tau_{S}\left(\pi\left(\pi\left(x, \tau_{S}(x)\right), r\right)\right)=r .
$$

Thus $r=\ell+\tau_{S}(x)$ is the unique time such that $\pi\left(x, \ell+\tau_{S}(x)\right) \in S_{\ell}$.

Moreover, as $\pi\left(x, \tau_{S}(x)\right) \in S$ and $S$ is a section, we have $F\left(x, \mathbb{R}_{+}\right) \cap S=\emptyset$, consequently, $F\left(x, \mathbb{R}_{+}\right) \bigcap S_{\ell}=\emptyset$.

Case 2: $x \in F\left(S_{\ell},(0, \ell]\right)$.

Subcase 2.1: $x \in F\left(S_{\ell}, \ell\right)$.

We have $\pi(x, \ell) \in S_{\ell}$, and, hence, there is $z_{x} \in S$ such that $\pi(x, \ell)=\pi\left(z_{x}, \ell\right)$.

We claim that $x \in S$, i.e., $\tau_{S}(x)=0$. In fact, suppose for a contradiction that $\tau_{S}(x)>0$. If $x \in F(S,(0, \infty))$, that is, $\pi\left(x, \tau_{S}(x)\right) \in S$, then $\pi\left(x, \tau_{S}(x)+\ell\right) \in S_{\ell}$. Thus, there is $x_{1} \in S$ such that 
$\pi\left(x, \tau_{S}(x)+\ell\right)=\pi\left(x_{1}, \ell\right)$. Then

$$
\ell=\tau_{S}\left(\pi\left(x_{1}, \ell\right)\right)=\tau_{S}\left(\pi\left(x, \tau_{S}(x)+\ell\right)\right)=\tau_{S}\left(\pi\left(z_{x}, \ell+\tau_{S}(x)\right)\right)=\ell+\tau_{S}(x),
$$

which is an absurd as $\tau_{S}(x)>0$. On the other side, if $x \in \pi(S,(0, \infty))$ then $\pi\left(v_{x}, \tau_{S}(x)\right)=x$ for some $v_{x} \in S$. Thus, $\pi\left(v_{x}, \tau_{S}(x)+\ell\right)=\pi(x, \ell)=\pi\left(z_{x}, \ell\right)$, which also leads us to the contradiction $\tau_{S}(x)+\ell=\ell$.

Hence, $x \in S$ and this shows that $\ell>0$ is the unique time such that $\pi(x, \ell) \in S_{\ell}$, because if $t_{1} \neq \ell$ is such that $\pi\left(x, t_{1}\right) \in S_{\ell}$ then taking $y \in S$ such that $\pi\left(x, t_{1}\right)=\pi(y, \ell)$, we get $t_{1}=$ $\tau_{S}\left(\pi\left(x, t_{1}\right)\right)=\tau_{S}(\pi(y, \ell))=\ell$, a contradiction.

Here, $F\left(x, \mathbb{R}_{+}\right) \bigcap S_{\ell}=\emptyset$ as $F(x,(0, \infty)) \bigcap S=\emptyset$.

Subcase 2.2: $x \in F\left(S_{\ell},(0, \ell)\right)$.

Here, there are $w \in S_{\ell}$ and $0<r<\ell$ such that $\pi(x, r)=w$. There is a unique $z_{w} \in S$ such that $\pi\left(z_{w}, \ell\right)=w$. Note that $\tau_{S}(w)=\ell$.

We claim that $x \in \pi\left(S, \mathbb{R}_{+}\right)$. Suppose to the contrary that $\pi\left(x, \tau_{S}(x)\right) \in S$. In this way,

$$
\tau_{S}\left(\pi\left(x, \tau_{S}(x)+\ell\right)\right)=\ell .
$$

But $\tau_{S}\left(\pi\left(x, \tau_{S}(x)+\ell\right)\right)=\tau_{S}\left(\pi\left(\pi(x, r), \tau_{S}(x)+\ell-r\right)\right)=\tau_{S}\left(\pi\left(z_{w}, \tau_{S}(x)+2 \ell-r\right)\right)=\tau_{S}(x)+2 \ell-r$. Consequently,

$$
\ell+\tau_{S}(x)=r
$$

which is a contradiction as $r<\ell$. Hence, $x \in \pi\left(S, \mathbb{R}_{+}\right)$, and since $S$ is a section, there is a unique $z_{x} \in S$ such that $\pi\left(z_{x}, \tau_{S}(x)\right)=x$. As a consequence, we conclude that

$$
\pi\left(z_{x}, \tau_{S}(x)+r\right)=\pi(x, r)=w=\pi\left(z_{w}, \ell\right) .
$$

Thus, $\tau_{S}(x)+r=\tau_{S}\left(\pi\left(z_{x}, \tau_{S}(x)+r\right)\right)=\tau_{S}\left(\pi\left(z_{w}, \ell\right)\right)=\ell$. This shows that $r=\ell-\tau_{S}(x)$ is the unique time such that $\pi\left(x, \ell-\tau_{S}(x)\right) \in S_{\ell}$, because if $t_{2} \geqslant 0$ is such that $\pi\left(x, t_{2}\right) \in S_{\ell}$ then taking $y_{1}, y_{2} \in S$ such that $\pi\left(x, t_{2}\right)=\pi\left(y_{1}, \ell\right)$ and $\pi\left(x, \ell-\tau_{S}(x)\right)=\pi\left(y_{2}, \ell\right)$, we get $2 \ell-\tau_{S}(x)=\tau_{S}\left(\pi\left(y_{1}, 2 \ell-\right.\right.$ $\left.\left.\tau_{S}(x)\right)\right)=\tau_{S}\left(\pi\left(x, t_{2}+\ell-\tau_{S}(x)\right)\right)=\tau_{S}\left(\pi\left(y_{2}, \ell+t_{2}\right)\right)=t_{2}+\ell$, that is, $t_{2}=\ell-\tau_{S}(x)$.

If there exists $a \in F\left(x, \mathbb{R}_{+}\right) \cap S_{\ell}$ then $\pi(a, s)=x$ and $a=\pi(b, \ell)$ for some $s \in \mathbb{R}_{+}$and $b \in S$. Now, using the fact that $\tau_{S}(\pi(x, r))=\tau_{S}(w)=\ell$, we get

$$
\ell=\tau_{S}(\pi(x, r))=\tau_{S}(\pi(b, s+r+\ell))=s+r+\ell,
$$


which is a contradiction. Hence, $F\left(x, \mathbb{R}_{+}\right) \cap S_{\ell}=\emptyset$.

Case 3: $x \in \pi\left(S_{\ell}, \mathbb{R}_{+}\right)$.

There are $w \in S_{\ell}$ and $t \in \mathbb{R}_{+}$such that $x=\pi(w, t)$. Since $S_{\ell}=\pi(S, \ell)$, there is $w_{s} \in S$ such that $w=\pi\left(w_{s}, \ell\right)$.

Suppose there are $v \in S_{\ell}$ and $r \in \mathbb{R}_{+}$such that $\pi(v, r)=x$. As before, one can find $v_{s} \in S$ with $v=\pi\left(v_{s}, \ell\right)$. Thus, $\pi\left(w_{s}, \ell+t\right)=x=\pi\left(v_{s}, \ell+r\right)$. But, as $S$ is a section we conclude that $w_{s}=v_{s}$ and $t=r$. Hence, there are unique $w \in S_{\ell}$ and $t \in \mathbb{R}_{+}$such that $\pi(w, t)=x$. Moreover, since $\pi\left(w_{s}, \ell+t\right)=x$ we get $\tau_{S}(x)=\ell+t$, i.e., $t=\tau_{S}(x)-\ell$.

If $t>0$ we still need to show that $\pi\left(x, \mathbb{R}_{+}\right) \bigcap S_{\ell}=\emptyset$. Suppose to the contradiction that there exists $z \in \pi\left(x, \mathbb{R}_{+}\right) \cap S_{\ell}$. Then $z=\pi\left(x, t_{1}\right)=\pi\left(z_{s}, \ell\right)$ for some $t_{1} \in \mathbb{R}_{+}$and $z_{s} \in S$. Recall that $x=\pi\left(w_{s}, t+\ell\right)$ with $w_{s} \in S$. Thus,

$$
\ell=\tau_{S}\left(\pi\left(z_{s}, \ell\right)\right)=\tau_{S}\left(\pi\left(x, t_{1}\right)\right)=\tau_{S}\left(\pi\left(w_{s}, t+t_{1}+\ell\right)\right)=t+t_{1}+\ell
$$

which contradicts the fact that $t>0$. Hence, $\pi\left(x, \mathbb{R}_{+}\right) \cap S_{\ell}=\emptyset$.

If $t=0\left(x=w=\pi\left(w_{s}, \ell\right)\right)$, we need to verify that $\pi(x,(0, \infty)) \cap S_{\ell}=\emptyset$ and that $F(x,(0, \infty)) \cap S_{\ell}=$ $\emptyset$. For the first equality, we can use the proof of the previous case $t>0$ and get a contradiction in (3.1.2) with $t=0$ and $t_{1}>0$. For the second equality, if there is $a \in F(x,(0, \infty)) \cap S_{\ell}$ then $\pi\left(a, t_{2}\right)=x$ and $a=\pi(b, \ell)$ for some $t_{2}>0$ and $b \in S$, which implies $\ell+t_{2}=\tau_{S}\left(\pi\left(b, \ell+t_{2}\right)\right)=$ $\tau_{S}(x)=\tau_{S}\left(\pi\left(w_{s}, \ell\right)\right)=\ell$, a contradiction.

In conclusion, $S_{\ell}$ is a section. Looking to the proof, the section function $\tau_{S_{\ell}}$ is characterized by

$$
\tau_{S_{\ell}}(x)=\left\{\begin{array}{rll}
\ell+\tau_{S}(x) & \text { if } & x \in F\left(S_{\ell},(\ell, \infty)\right), \\
\ell-\tau_{S}(x) & \text { if } & x \in F\left(S_{\ell},(0, \ell]\right), \\
-\ell+\tau_{S}(x) & \text { if } & x \in \pi\left(S_{\ell}, \mathbb{R}_{+}\right),
\end{array}\right.
$$

which is continuous as $\tau_{S}$ is continuous.

Lemma 3.1.5. Let $S$ be a section for $\left(X, \pi, \mathbb{R}_{+}\right), x \in X$ and $t \in \mathbb{R}_{+}$.

(a) If $x \in F\left(S, \mathbb{R}_{+}\right)$and $t<\tau_{S}(x)$ then $t=\tau_{S}(x)-\tau_{S}(\pi(x, t))$.

(b) If $x \in F\left(S, \mathbb{R}_{+}\right)$and $t \geqslant \tau_{S}(x)$ then $t=\tau_{S}(\pi(x, t))+\tau_{S}(x)$.

(c) If $x \in \pi\left(S, \mathbb{R}_{+}\right)$then $t=\tau_{S}(\pi(x, t))-\tau_{S}(x)$. 
Proof. (a) Let $x \in F\left(S, \mathbb{R}_{+}\right)$and $t<\tau_{S}(x)$. Then $\pi(x, t) \in F\left(S, \mathbb{R}_{+}\right)$. Thus, there is $w_{x} \in S$ such that $\pi\left(\pi(x, t), \tau_{S}(\pi(x, t))\right)=w_{x}$. This implies that

$$
\tau_{S}\left(\pi\left(x, t+\tau_{S}(\pi(x, t))\right)\right)=\tau_{S}\left(w_{x}\right)=0 .
$$

As a consequence, we have $\pi\left(x, t+\tau_{S}(\pi(x, t))\right) \in S$, that is, $\tau_{S}(x)=t+\tau_{S}(\pi(x, t))$.

(b) Let $x \in F\left(S, \mathbb{R}_{+}\right)$and $t \geqslant \tau_{S}(x)$. There is $z_{x} \in S$ such that $\pi\left(x, \tau_{S}(x)\right)=z_{x}$. Since $t \geqslant \tau_{S}(x)$, we get

$$
\tau_{S}(\pi(x, t))=\tau_{S}\left(\pi\left(\pi\left(x, \tau_{S}(x)\right), t-\tau_{S}(x)\right)\right)=\tau_{S}\left(\pi\left(z_{x}, t-\tau_{S}(x)\right)\right)=t-\tau_{S}(x) .
$$

(c) Let $x \in \pi\left(S, \mathbb{R}_{+}\right)$and $t \in \mathbb{R}_{+}$. There exists $z_{x} \in S$ such that $x=\pi\left(z_{x}, \tau_{S}(x)\right)$. Thus, $\tau_{S}(\pi(x, t))=\tau_{S}\left(\pi\left(z_{x}, \tau_{S}(x)+t\right)\right)=\tau_{S}(x)+t$.

\subsection{Parallelizability versus sections}

This section concerns the relationship between parallelizable semidynamical systems and the existence of sections. In Theorem 3.2.1, we show that a parallelizable semidynamical system admits a section with continuous function section. Sufficient conditions to obtain parallelizability are presented in Theorem 3.2.2 and Corollary 3.2.2.

The first result of this section characterizes the function $h$ presented in Definition 3.1.2 using a section function.

Lemma 3.2.1. Let $S$ be a section for $\left(X, \pi, \mathbb{R}_{+}\right)$with section function $\tau_{S}$.

(a) The function $g: X \rightarrow S \times \mathbb{R}_{+}$given by

$$
g(x)= \begin{cases}\left(\pi\left(x, \tau_{S}(x)\right), \tau_{S}(x)\right) & \text { if } x \in F\left(S, \mathbb{R}_{+}\right), \\ \left(z_{x}, \tau_{S}(x)\right) & \text { if } x \in \pi\left(S, \mathbb{R}_{+}\right),\end{cases}
$$

where $z_{x}$ is the unique element in $S$ such that $\pi\left(z_{x}, \tau_{S}(x)\right)=x$, satisfies the conditions $(i)$ and (ii) of Definition 3.1.2.

(b) If $\left(X, \pi, \mathbb{R}_{+}\right)$is parallelizable with base $S$ and continuous function $h: X \rightarrow S \times \mathbb{R}_{+}$satisfying conditions $(i)$ and $(i i)$ of Definition 3.1.2, then $h(x)=g(x)$ for all $x \in X$. Moreover, the section function $\tau_{S}$ is continuous on $X$. 
Proof. (a) Note that $g$ is well defined as $S$ is a section. Furthermore,

$$
g(\pi(w, t))=\left(z_{\pi(w, t)}, \tau_{S}(\pi(w, t))\right)=(w, t) \quad \text { for all } \quad w \in S \quad \text { and } \quad t \in \mathbb{R}_{+},
$$

and

$$
g(x)=\left(\pi\left(x, \tau_{S}(x)\right), \tau_{S}(x)\right)=(w, t) \quad \text { for all } \quad x \in F(w, t), \text { with } w \in S \text { and } t \in \mathbb{R}_{+} .
$$

Hence, $g$ satisfies the conditions $(i)$ and $(i i)$ of Definition 3.1.2.

(b) By Lemma 3.1.1, $h(x)=g(x)$ for all $x \in X$. Now, let us prove that $\tau_{S}$ is continuous on $X$. Let $x \in X$ and $\left\{x_{n}\right\}_{n \in \mathbb{N}} \subset X$ be a sequence such that $x_{n} \stackrel{n \rightarrow \infty}{\longrightarrow} x$. We have some cases to consider:

Case 1: $x \in F(S,(0, \infty))$.

By Lemma 3.1.2, we may assume that $\left\{x_{n}\right\}_{n \in \mathbb{N}} \subset F(S,(0, \infty))$. Thus,

$$
\left(\pi\left(x_{n}\right), \tau_{S}\left(x_{n}\right), \tau_{S}\left(x_{n}\right)\right)=h\left(x_{n}\right) \stackrel{n \rightarrow \infty}{\longrightarrow} h(x)=\left(\pi\left(x, \tau_{S}(x)\right), \tau_{S}(x)\right),
$$

that is, $\tau_{S}\left(x_{n}\right) \stackrel{n \rightarrow \infty}{\longrightarrow} \tau_{S}(x)$.

Case 2: $x \in \pi(S,(0, \infty))$.

By Lemma 3.1.2, we may assume that $\left\{x_{n}\right\}_{n \in \mathbb{N}} \subset \pi(S,(0, \infty))$. Then

$$
\left(z_{x_{n}}, \tau_{S}\left(x_{n}\right)\right)=h\left(x_{n}\right) \stackrel{n \rightarrow \infty}{\longrightarrow} h(x)=\left(z_{x}, \tau_{S}(x)\right),
$$

that is, $\tau_{S}\left(x_{n}\right) \stackrel{n \rightarrow \infty}{\longrightarrow} \tau_{S}(x)$.

Case 3: $x \in S$.

In this case, $\tau_{S}(x)=0$ and $h(x)=(x, 0)$. If $\left\{x_{n}\right\}_{n \in \mathbb{N}}$ admits a subsequence $\left\{x_{n_{k}}\right\}_{k \in \mathbb{N}} \subset$ $F\left(S, \mathbb{R}_{+}\right)$then

$$
\left(\pi\left(x_{n_{k}}\right), \tau_{S}\left(x_{n_{k}}\right), \tau_{S}\left(x_{n_{k}}\right)\right)=h\left(x_{n_{k}}\right) \stackrel{k \rightarrow \infty}{\longrightarrow} h(x)=(x, 0),
$$

which implies $\tau_{S}\left(x_{n_{k}}\right) \stackrel{k \rightarrow \infty}{\longrightarrow} \tau_{S}(x)$.

Also, if $\left\{x_{n}\right\}_{n \in \mathbb{N}}$ admits a subsequence $\left\{x_{m_{k}}\right\}_{k \in \mathbb{N}} \subset \pi\left(S, \mathbb{R}_{+}\right)$then

$$
\left(z_{x_{m_{k}}}, \tau_{S}\left(x_{m_{k}}\right)\right)=h\left(x_{m_{k}}\right) \stackrel{k \rightarrow \infty}{\longrightarrow} h(x)=(x, 0)
$$

which implies $\tau_{S}\left(x_{m_{k}}\right) \stackrel{k \rightarrow \infty}{\longrightarrow} \tau_{S}(x)$.

Hence, $\tau_{S}\left(x_{n}\right) \stackrel{n \rightarrow \infty}{\longrightarrow} \tau_{S}(x)$.

In conclusion, $\tau_{S}$ is continuous on $X$. 
Theorem 3.2.1. Let $\left(X, \pi, \mathbb{R}_{+}\right)$be parallelizable with base $S$. Then $S$ is a section with section function $\tau_{S}$ continuous on $X$.

Proof. Since $\left(X, \pi, \mathbb{R}_{+}\right)$is parallelizable, there exists a continuous function $h: X \rightarrow S \times \mathbb{R}_{+}$satisfying conditions $(i)$ and (ii) of Definition 3.1.2, where $X=F\left(S, \mathbb{R}_{+}\right) \cup \pi\left(S, \mathbb{R}_{+}\right)$.

Let $x \in X$. Assume at first that $x \in S$. If $\pi(x, t)=y \in S$ then

$$
(y, 0)=h(y)=h(\pi(x, t))=(x, t),
$$

that is, $y=x$ and $t=0$. Analogously, if $z \in S$ is such that $\pi(z, t)=x$ then we obtain $z=x$ and $t=0$. Hence, $F(x,(0, \infty)) \bigcap S=\emptyset$ and $\pi(x,(0, \infty)) \bigcap S=\emptyset$ provided $x \in S$.

Now, we assume that $x \in F(S,(0, \infty))$. Suppose to the contrary that there are $\tau_{1}, \tau_{2} \in(0, \infty)$ such that $\pi\left(x, \tau_{1}\right) \in S$ and $\pi\left(x, \tau_{2}\right) \in S$. We may assume that $\tau_{1}<\tau_{2}$. Then

$$
\left(\pi\left(x, \tau_{2}\right), 0\right)=h\left(\pi\left(x, \tau_{2}\right)\right)=h\left(\pi\left(\pi\left(x, \tau_{1}\right), \tau_{2}-\tau_{1}\right)\right)=\left(\pi\left(x, \tau_{1}\right), \tau_{2}-\tau_{1}\right),
$$

which shows that $\tau_{1}=\tau_{2}$, a contradiction. Hence, there is a unique $t_{x}>0$ such that $\pi\left(x, t_{x}\right) \in S$. By the previous case, we have $F\left(x, \mathbb{R}_{+}\right) \cap S=\emptyset$.

Lastly, assume that $x \in \pi(S,(0, \infty))$. Suppose there are $z_{1}, z_{2} \in S$ and $\tau_{1}, \tau_{2}>0$ such that $\pi\left(z_{1}, \tau_{1}\right)=x$ and $\pi\left(z_{2}, \tau_{2}\right)=x$. Thus

$$
\left(z_{1}, \tau_{1}\right)=h\left(\pi\left(z_{1}, \tau_{1}\right)\right)=h(x)=h\left(\pi\left(z_{2}, \tau_{2}\right)\right)=\left(z_{2}, \tau_{2}\right),
$$

that is, $z_{1}=z_{2}$ and $t_{1}=t_{2}$. Thus, there are unique $z_{x} \in S$ and $t_{x}>0$ such that $\pi\left(z_{x}, t_{x}\right)=x$. By the first case, we have $\pi\left(x, \mathbb{R}_{+}\right) \cap S=\emptyset$.

Therefore, $S$ is a section for $\left(X, \pi, \mathbb{R}_{+}\right)$. Let $\tau_{S}: X \rightarrow \mathbb{R}_{+}$be the associated section function as presented in (3.1.1). The continuity of $\tau_{S}$ follows by Lemma 3.2.1, $(b)$.

As shown in the proof of Lemma 3.2.1, when $S$ is a section for $\left(X, \pi, \mathbb{R}_{+}\right)$with continuous section function $\tau_{S}$, the function $g: X \rightarrow S \times \mathbb{R}_{+}$defined in (3.2.1) satisfies the conditions $(i)$ and $(i i)$ of Definition 3.1.2. However, if $\operatorname{dim} X=\infty$ then we cannot conclude that $\left(X, \pi, \mathbb{R}_{+}\right)$is parallelizable, since we cannot assure that $g$ is continuous on $X$ even $\tau_{S}$ is continuous. In the sequel, we establish some sufficient conditions to obtain the converse of Theorem 3.2.1.

Theorem 3.2.2. Assume that $\left(X, \pi, \mathbb{R}_{+}\right)$admits a compact section $S$ with a continuous section function $\tau_{S}$. Then $\left(X, \pi, \mathbb{R}_{+}\right)$is parallelizable. 
Proof. Since $S$ is a section, we have $X=F\left(S, \mathbb{R}_{+}\right) \cup \pi\left(S, \mathbb{R}_{+}\right)$. By Lemma 3.2.1, the function $g: X \rightarrow S \times \mathbb{R}_{+}$given by

$$
g(x)= \begin{cases}\left(\pi\left(x, \tau_{S}(x)\right), \tau_{S}(x)\right) & \text { if } \quad x \in F\left(S, \mathbb{R}_{+}\right) \\ \left(z_{x}, \tau_{S}(x)\right) & \text { if } x \in \pi\left(S, \mathbb{R}_{+}\right)\end{cases}
$$

where $z_{x}$ is the unique element in $S$ such that $\pi\left(z_{x}, \tau_{S}(x)\right)=x$, satisfies the conditions $(i)$ and (ii) of Definition 3.1.2. It remains to show the continuity of $g$. Since $\pi$ and $\tau_{S}$ are continuous, we need just to prove that the function $f: \pi\left(S, \mathbb{R}_{+}\right) \rightarrow S$ given by $f(x)=z_{x}$ is continuous. Indeed, let $x \in \pi\left(S, \mathbb{R}_{+}\right)$and $\left\{x_{n}\right\}_{n \in \mathbb{N}} \subset \pi\left(S, \mathbb{R}_{+}\right)$be a sequence such that $x_{n} \stackrel{n \rightarrow \infty}{\longrightarrow} x$. Since $S$ is compact, we may assume that $f\left(x_{n}\right)=z_{x_{n}} \stackrel{n \rightarrow \infty}{\longrightarrow} w$ for some $w \in S$. Recall that $\pi\left(z_{x_{n}}, \tau_{S}\left(x_{n}\right)\right)=x_{n}, n \in \mathbb{N}$. Then

$$
\pi\left(z_{x}, \tau_{S}(x)\right)=x=\lim _{n \rightarrow \infty} x_{n}=\lim _{n \rightarrow \infty} \pi\left(z_{x_{n}}, \tau_{S}\left(x_{n}\right)\right)=\pi\left(w, \tau_{S}(x)\right)
$$

which implies that $w=z_{x}$ as $S$ is a section. Hence, $f\left(x_{n}\right) \stackrel{n \rightarrow \infty}{\longrightarrow} f(x)$. Since $\pi(S,(0, \infty))$ and $F(S,(0, \infty))$ are open sets by Lemma 3.1.3, and $\tau_{S}\left(x_{n}\right) \stackrel{n \rightarrow \infty}{\longrightarrow} 0$ provided $x_{n} \stackrel{n \rightarrow \infty}{\longrightarrow} x=z_{x} \in S$, we conclude that $g$ is continuous on $X$.

Definition 3.2.1. A semidynamical system $\left(X, \pi, \mathbb{R}_{+}\right)$is called completely continuous, if for every bounded subset $B \subset X$ then there exists $t_{0}=t_{0}(B)>0$ such that $\pi\left(B, t_{0}\right)$ is relatively compact.

Using Lemma 3.1.4, we have the following consequence of Theorem 3.2.2.

Corollary 3.2.1. Assume that $\left(X, \pi, \mathbb{R}_{+}\right)$is completely continuous and admits a bounded section $S$ with a continuous section function $\tau_{S}$. Then $\left(X, \pi, \mathbb{R}_{+}\right)$is parallelizable.

Next, we introduce a special condition which will be useful to obtain the continuity of a section function.

Definition 3.2.2. A section $S$ satisfies the condition $(T)$ if for every $z \in S$ and every sequence $\left\{z_{n}\right\}_{n \in \mathbb{N}} \subset X$ such that $z_{n} \stackrel{n \rightarrow \infty}{\longrightarrow} z$ then $\tau_{S}\left(z_{n}\right) \stackrel{n \rightarrow \infty}{\longrightarrow} 0$.

In Lemma 3.2.2 below, we prove the openness of $F(S,(0, \infty))$ using the condition $(T)$.

Lemma 3.2.2. Let $\left(X, \pi, \mathbb{R}_{+}\right)$admit a section $S$ satisfying condition $(T)$. Then $F(S,(0, \infty))$ is open in $X$. 
Proof. Suppose to the contrary that $F(S,(0, \infty))$ is not open. In this way, there are $x \in F(S,(0, \infty))$ and a sequence $x_{n} \stackrel{n \rightarrow \infty}{\longrightarrow} x$ such that $x_{n} \notin F(S,(0, \infty)), n \in \mathbb{N}$. Since $S$ is a section, we have $\left\{x_{n}\right\}_{n \in \mathbb{N}} \subset$ $\pi(S,[0, \infty))$. Let $z_{x} \in S$ be such that $\pi\left(x, \tau_{S}(x)\right)=z_{x}$ and let $v_{n} \in S$ be such that $\pi\left(v_{n}, \tau_{S}\left(x_{n}\right)\right)=x_{n}$, $n \in \mathbb{N}$. Since

$$
\pi\left(v_{n}, \tau_{S}\left(x_{n}\right)+\tau_{S}(x)\right)=\pi\left(x_{n}, \tau_{S}(x)\right) \stackrel{n \rightarrow \infty}{\longrightarrow} \pi\left(x, \tau_{S}(x)\right)=z_{x} \in S,
$$

it follows by condition $(T)$ that $\tau_{S}\left(\pi\left(v_{n}, \tau_{S}\left(x_{n}\right)+\tau_{S}(x)\right)\right) \stackrel{n \rightarrow \infty}{\longrightarrow} 0$. But

$$
\tau_{S}\left(\pi\left(v_{n}, \tau_{S}\left(x_{n}\right)+\tau_{S}(x)\right)\right)=\tau_{S}\left(x_{n}\right)+\tau_{S}(x)
$$

which leads us to a contradiction as $\tau_{S}(x)>0(x \notin S)$. Hence, $F(S,(0, \infty))$ is open in $X$.

As defined in Definition 2.1.4, consider the set-valued map $F: X \times \mathbb{R}_{+} \rightarrow X$ given by $F(x, t)=\{y \in X: \pi(y, t)=x\}$.

Definition 3.2.3. The map $F: X \times \mathbb{R}_{+} \rightarrow X$ is said to be upper semicontinuous at $(x, t) \in X \times$ $\mathbb{R}_{+}$, if for all sequences $\left\{x_{n}\right\}_{n \in \mathbb{N}} \subset X$ and $\left\{t_{n}\right\}_{n \in \mathbb{N}} \subset \mathbb{R}_{+}$such that $x_{n} \stackrel{n \rightarrow \infty}{\longrightarrow} x$ and $t_{n} \stackrel{n \rightarrow \infty}{\longrightarrow} t$ then $d_{H}\left(F\left(x_{n}, t_{n}\right), F(x, t)\right) \stackrel{n \rightarrow \infty}{\longrightarrow} 0$. The map $F$ is upper semicontinuous in $A \subset X \times \mathbb{R}_{+}$, if it is upper semicontinuous at each point $(x, t) \in A$.

Theorem 3.2.3 below provides sufficient conditions to obtain the continuity of a section function.

Theorem 3.2.3. Assume that $\left(X, \pi, \mathbb{R}_{+}\right)$admits a section $S$ satisfying condition $(T)$. If $F$ is upper semicontinuous on $\pi\left(S, \mathbb{R}_{+}\right)$, then the section function $\tau_{S}$ is continuous on $X$.

Proof. Suppose to the contrary that $\tau_{S}$ is discontinuous at some $x \in X$. Then there are $\varepsilon_{0}>0$ and a sequence $\left\{x_{n}\right\}_{n \in \mathbb{N}} \subset X$ with $x_{n} \stackrel{n \rightarrow \infty}{\longrightarrow} x$ such that

$$
\left|\tau_{S}\left(x_{n}\right)-\tau_{S}(x)\right| \geqslant \varepsilon_{0} \quad \text { for all } n \in \mathbb{N}
$$

Case 1: $x \in F(S,(0, \infty))$.

In this case, $\pi\left(x, \tau_{S}(x)\right) \in S$ with $\tau_{S}(x)>0$. Since $\pi\left(x_{n}, \tau_{S}(x)\right) \stackrel{n \rightarrow \infty}{\longrightarrow} \pi\left(x, \tau_{S}(x)\right) \in S$, it follows by condition $(T)$ that $\tau_{S}\left(\pi\left(x_{n}, \tau_{S}(x)\right)\right) \stackrel{n \rightarrow \infty}{\longrightarrow} 0$. Now, Lemma 3.2.2 assures that $F(S,(0, \infty))$ is open, consequently we may assume that $\left\{x_{n}\right\}_{n \in \mathbb{N}} \subset F(S,(0, \infty))$ and $\pi\left(x_{n}, \tau_{S}\left(x_{n}\right)\right) \in S$ for all $n \in \mathbb{N}$. 
If $\left\{x_{n}\right\}_{n \in \mathbb{N}}$ admits a subsequence, denoted by the same, such that $\tau_{S}\left(x_{n}\right) \leqslant \tau_{S}(x)$ then using Lemma 3.1.5, item $(b)$, we have

$$
\tau_{S}\left(\pi\left(x_{n}, \tau_{S}(x)\right)\right)=\tau_{S}(x)-\tau_{S}\left(x_{n}\right) \stackrel{n \rightarrow \infty}{\longrightarrow} 0 .
$$

If $\left\{x_{n}\right\}_{n \in \mathbb{N}}$ admits a subsequence, denoted by the same, such that $\tau_{S}\left(x_{n}\right)>\tau_{S}(x)$ then using Lemma 3.1.5, item $(a)$, we have

$$
\tau_{S}\left(\pi\left(x_{n}, \tau_{S}(x)\right)\right)=\tau_{S}\left(x_{n}\right)-\tau_{S}(x) \stackrel{n \rightarrow \infty}{\longrightarrow} 0
$$

In both cases above, we get a contradiction as inequality (3.2.3) holds.

Case 2: $x \in \pi\left(S, \mathbb{R}_{+}\right)$.

There is $z_{x} \in S$ such that $x=\pi\left(z_{x}, \tau_{S}(x)\right)$. Since $S$ is a section, $F\left(x, \tau_{S}(x)\right)=\left\{z_{x}\right\}$. Now, let $w_{n} \in F\left(x_{n}, \tau_{S}(x)\right), n \in \mathbb{N}$. Since $d_{H}\left(F\left(x_{n}, \tau_{S}(x)\right), F\left(x, \tau_{S}(x)\right)\right) \stackrel{n \rightarrow \infty}{\longrightarrow} 0$ and $F\left(x, \tau_{S}(x)\right)=\left\{z_{x}\right\}$, we have $w_{n} \stackrel{n \rightarrow \infty}{\longrightarrow} z_{x} \in S$. By condition $(\mathrm{T}), \tau_{S}\left(w_{n}\right) \stackrel{n \rightarrow \infty}{\longrightarrow} 0$.

We can decompose $\left\{w_{n}\right\}_{n \in \mathbb{N}}$ into $\left\{w_{n}\right\}_{n \in \mathbb{N}}=\left\{w_{k}\right\}_{k \in \mathbb{N}_{1}} \cup\left\{w_{\ell}\right\}_{\ell \in \mathbb{N}_{2}}$, where $\mathbb{N}=\mathbb{N}_{1} \bigcup \mathbb{N}_{2}$,

$$
\left\{w_{k}\right\}_{k \in \mathbb{N}_{1}} \subset F(S,(0, \infty)) \text { and }\left\{w_{\ell}\right\}_{\ell \in \mathbb{N}_{2}} \subset \pi\left(S, \mathbb{R}_{+}\right) \text {. }
$$

Let us assume that $\mathbb{N}_{1}$ and $\mathbb{N}_{2}$ are countably infinite sets. By Lemma 3.1.5, items $(a)$ and $(b)$, we have

$$
\tau_{S}\left(x_{k}\right)=\tau_{S}\left(\pi\left(w_{k}, \tau_{S}(x)\right)\right)=\mid \tau_{S}(x)-\tau_{S}\left(w_{k}\right) \stackrel{k \rightarrow \infty}{\longrightarrow} \tau_{S}(x),
$$

and by item $(c)$, we have

$$
\tau_{S}\left(x_{\ell}\right)=\tau_{S}\left(\pi\left(w_{\ell}, \tau_{S}(x)\right)\right)=\tau_{S}(x)+\tau_{S}\left(w_{\ell}\right) \stackrel{\ell \rightarrow \infty}{\longrightarrow} \tau_{S}(x)
$$

which contradicts inequality (3.2.3).

Therefore, $\tau_{S}$ is continuous on $X$.

Following the proof of Theorem 3.2.2 and the previous results, we have the next corollary. We just observe that the continuity of $f$ in the proof of Theorem 3.2.2 is obtained using the upper semicontinuity of $F$.

Corollary 3.2.2. Assume that $\left(X, \pi, \mathbb{R}_{+}\right)$admits a section $S$ satisfying condition $(T)$. If $F$ is upper semicontinuous on $\pi\left(S, \mathbb{R}_{+}\right)$, then $\left(X, \pi, \mathbb{R}_{+}\right)$is parallelizable. 
We end this section with the following result concerning stationary points and periodic points.

Theorem 3.2.4. Let $\left(X, \pi, \mathbb{R}_{+}\right)$be parallelizable with section $S$. Then there exist neither stationary points nor periodic points in $\left(X, \pi, \mathbb{R}_{+}\right)$.

Proof. Suppose to the contrary that $x$ is a periodic point with period $T>0$. If $x \in F\left(S, \mathbb{R}_{+}\right)$then $\pi\left(x, \tau_{S}(x)\right) \in S$. Since $x$ is periodic we have $\pi\left(x, \tau_{S}(x)+T\right) \in S$, which implies $\tau_{S}(x)=\tau_{S}(x)+T$ as $S$ is a section. But this is a contradiction because $T \neq 0$.

On the other side, if $x \in \pi\left(S, \mathbb{R}_{+}\right)$, then there is $z_{x} \in S$ such that $\pi\left(z_{x}, \tau_{S}(x)\right)=x=$ $\pi\left(z_{x}, \tau_{S}(x)+T\right)$, thus $T=0$, which is again a contradiction. In this way, there is no periodic point in $\left(X, \pi, \mathbb{R}_{+}\right)$.

By the definition of section, a parallelizable system $\left(X, \pi, \mathbb{R}_{+}\right)$cannot admit stationary points.

\subsection{Parallelizability versus dispersivity}

The relationship between parallelizable dynamical systems and dispersive dynamical systems was investigated in the early 60s in the paper (ANTOSIEWICZ; DUGUNDJI, 1960). Later, this relationship was studied by Hajek in (HáJEK, 1971) and by Egawa in (EGAWA, 1972).

Analogously to dynamical systems, we define the concept of dispersiveness for semidynamical systems using the positive prolongational limit set, see Definition 2.1.7.

Definition 3.3.1. A semidynamical system $\left(X, \pi, \mathbb{R}_{+}\right)$is called dispersive if $J^{+}(x)=\emptyset$ for all $x \in X$.

Example 3.3.1. Let $f: \mathbb{R}^{2} \rightarrow \mathbb{R}$ be a continuous function satisfying the following conditions: $f(x, y)>0$ whenever $(x, y) \neq\left(n, \frac{1}{n}\right), n \in \mathbb{N}$, and $f\left(n, \frac{1}{n}\right)=0$ for all $n \in \mathbb{N}$. Consider the set

$$
X=\mathbb{R}^{2} \backslash\left(\bigcup_{n \in \mathbb{N}} I_{n}\right),
$$

where $I_{n}=\left\{\left(x, \frac{1}{n}\right): x \in(-\infty, n]\right\}$ for each $n \in \mathbb{N}$. The system of differential equations

$$
\left\{\begin{array}{l}
\frac{d x}{d t}=f(x, y), \\
\frac{d y}{d t}=0,
\end{array}\right.
$$


generates a semidynamical system on $X=\mathbb{R}^{2} \backslash\left(\bigcup_{n \in \mathbb{N}} I_{n}\right)$, namely $\left(X, \pi, \mathbb{R}_{+}\right)$.

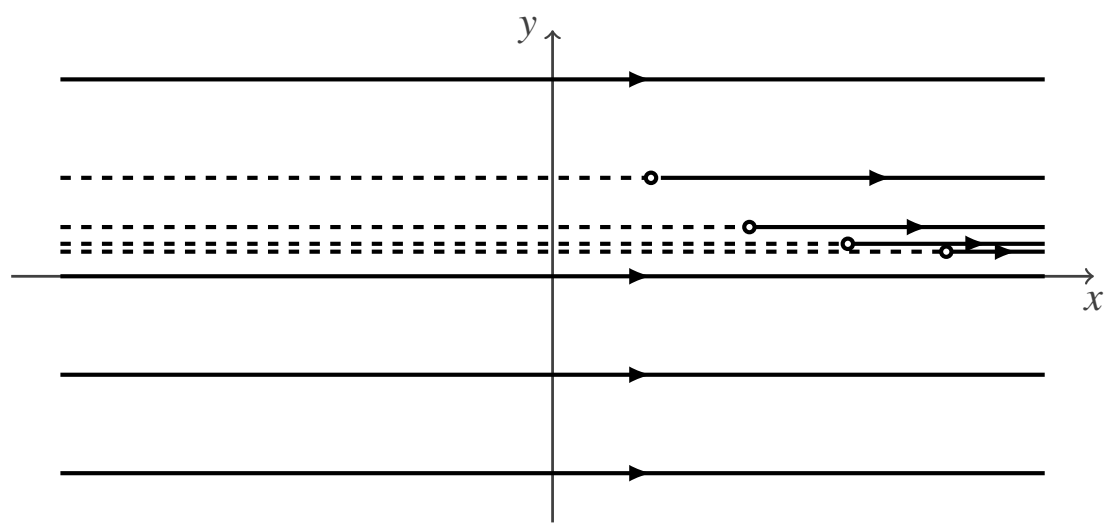

Figure 3.2: The positive trajectories of the semidynamical system $\left(X, \pi, \mathbb{R}_{+}\right)$.

Note that $J^{+}(z)=\emptyset$ for every $z=(x, y) \in X$. Hence, $\left(X, \pi, \mathbb{R}_{+}\right)$is dispersive. This example is known as the Bebutov's example and it is presented in (BHATIA; SZEGÖ, 2002) (see Chapter IV, 1.5.5).

In Theorem 3.3.1, we show that every parallelizable semidynamical system is dispersive.

Theorem 3.3.1. Let $\left(X, \pi, \mathbb{R}_{+}\right)$be parallelizable with section $S$. Then $\left(X, \pi, \mathbb{R}_{+}\right)$is dispersive.

Proof. Suppose to the contrary that there is $x \in X$ with $J^{+}(x) \neq \emptyset$ and let $y \in J^{+}(x)$. Then there are sequences $\left\{x_{n}\right\}_{n \in \mathbb{N}} \subset X$ and $\left\{t_{n}\right\}_{n \in \mathbb{N}} \subset \mathbb{R}_{+}$with $x_{n} \stackrel{n \rightarrow \infty}{\longrightarrow} x$ and $t_{n} \stackrel{n \rightarrow \infty}{\longrightarrow \infty}$ such that

$$
\pi\left(x_{n}, t_{n}\right) \stackrel{n \rightarrow \infty}{\longrightarrow} y .
$$

By Theorem 3.2.1 the function $\tau_{S}$ is continuous on $X$, which implies $\tau_{S}\left(x_{n}\right) \stackrel{n \rightarrow \infty}{\longrightarrow} \tau_{S}(x)$ and $\tau_{S}\left(\pi\left(x_{n}, t_{n}\right)\right) \stackrel{n \rightarrow \infty}{\longrightarrow} \tau_{S}(y)$.

We may assume that $\left\{x_{n}\right\}_{n \in \mathbb{N}}$ can be decomposed into $\left\{x_{n}\right\}_{n \in \mathbb{N}}=\left\{y_{k}\right\}_{k \in \mathbb{N}_{1}} \cup\left\{z_{\ell}\right\}_{\ell \in \mathbb{N}_{2}}$, where $\mathbb{N}=\mathbb{N}_{1} \cup \mathbb{N}_{2}$ (with $\mathbb{N}_{1}$ and $\mathbb{N}_{2}$ countably infinite sets),

$$
\left\{y_{k}\right\}_{k \in \mathbb{N}_{1}} \subset F(S,(0, \infty)) \text { and } \quad\left\{z_{\ell}\right\}_{\ell \in \mathbb{N}_{2}} \subset \pi(S,[0, \infty))
$$

Moreover, we may assume that $t_{k}>\tau_{S}\left(y_{k}\right)$ for all $k \in \mathbb{N}_{1}$. By Lemma 3.1.5, item $(b)$, we obtain

$$
t_{k}=\tau_{S}\left(\pi\left(y_{k}, t_{k}\right)\right)+\tau_{S}\left(y_{k}\right) \stackrel{k \rightarrow \infty}{\longrightarrow} \tau_{S}(y)+\tau_{S}(x),
$$


and by item $(c)$,

$$
t_{\ell}=\tau_{S}\left(\pi\left(z_{\ell}, t_{\ell}\right)\right)-\tau_{S}\left(z_{\ell}\right) \stackrel{\ell \rightarrow \infty}{\longrightarrow} \tau_{S}(y)-\tau_{S}(x),
$$

which is a contradiction as $t_{n} \stackrel{n \rightarrow \infty}{\longrightarrow} \infty$. Therefore, $J^{+}(x)=\emptyset$ for all $x \in X$.

Example 3.3.1 exhibits a semidynamical system that is dispersive. But this system is not parallelizable. Indeed, suppose to the contrary that the semidynamical system $\left(X, \pi, \mathbb{R}_{+}\right)$given in Example 3.3.1 is parallelizable. Let $S$ be its base. By Theorem 3.2.1, $S$ is a section with section function $\tau_{S}$ continuous on $X$. Let

$$
\{(x, 0): x \in \mathbb{R}\} \bigcap S=\left\{\left(x_{0}, 0\right)\right\}
$$

and, for each $n \in \mathbb{N}$,

$$
\left\{\left(x, \frac{1}{n}\right): x>n\right\} \bigcap S=\left\{\left(n+x_{n}, \frac{1}{n}\right)\right\},
$$

for some $x_{n}>0$. By continuity of $\tau_{S}$, there exists $\delta>0$ such that

$$
\left|\tau_{S}\left(x_{0}, s\right)\right|<\frac{1}{2}
$$

whenever $|s|<\delta$. Let $\left\{\lambda_{n}\right\}_{n \in \mathbb{N}} \subset \mathbb{R}_{+}$be a sequence such that $\lambda_{n} \stackrel{n \rightarrow \infty}{\longrightarrow} 0$. Now, let $m_{0}, n_{0} \in \mathbb{N}$ be such that $\frac{1}{m}+\lambda_{n}<\delta$ for all $n, m \geqslant n_{0}$.

Set $\left(p_{m}, \frac{1}{m}\right)=\pi\left(\left(m+x_{m}, \frac{1}{m}\right), 1\right), m \in \mathbb{N}$. Then

$$
\tau_{S}\left(p_{m}, \frac{1}{m}\right)=1 \quad \text { for all } \quad m \in \mathbb{N} .
$$

On the other hand, for each $m \in \mathbb{N}$, there exists $T_{m}>0$ such that

$$
d\left(\pi\left(\left(x_{0}, \frac{1}{m}+\lambda_{n}\right), T_{m}\right),\left(p_{m}, \frac{1}{m}\right)\right) \stackrel{n \rightarrow \infty}{\longrightarrow} 0 .
$$

Note that $T_{m} \stackrel{m \rightarrow \infty}{\longrightarrow} \infty$. Choose $m \geqslant m_{0}$ such that $T_{m}>2$. Using Lemma 3.1.5, we obtain

$$
\tau_{S}\left(\pi\left(\left(x_{0}, \frac{1}{m}+\lambda_{n}\right), T_{m}\right)\right)=T_{m} \pm \tau_{S}\left(x_{0}, \lambda_{n}+\frac{1}{m}\right)>2-\frac{1}{2}=\frac{3}{2}, \quad n \geqslant n_{0},
$$

which contradicts the continuity of $\tau_{S}$. Thus, $\left(X, \pi, \mathbb{R}_{+}\right)$is not parallelizable. Consequently, the converse of Theorem 3.3.1 does not hold in general. However, if a semidynamical system admits a compact section then the converse of Theorem 3.3.1 holds, as shown in the next result. 
Theorem 3.3.2. Let $\left(X, \pi, \mathbb{R}_{+}\right)$be dispersive. If $\left(X, \pi, \mathbb{R}_{+}\right)$admits a compact section $S$, then $\left(X, \pi, \mathbb{R}_{+}\right)$is parallelizable.

Proof. By Theorem 3.2.2, it is enough to show that $\tau_{S}$ is continuous on $X$. Suppose to the contrary that $\tau_{S}$ is discontinuous at some point $x \in X$. Then there are $\varepsilon_{0}>0$ and a sequence $\left\{x_{n}\right\}_{n \in \mathbb{N}} \subset X$ such that $x_{n} \stackrel{n \rightarrow \infty}{\longrightarrow} x$ and $\left|\tau_{S}\left(x_{n}\right)-\tau_{S}(x)\right| \geqslant \varepsilon_{0}$ for all $n \in \mathbb{N}$. Up to a subsequence, we may consider the following cases:

Case 1: $\left\{x_{n}\right\}_{n \in \mathbb{N}} \subset F\left(S, \mathbb{R}_{+}\right)$.

In this case, $\pi\left(x_{n}, \tau_{S}\left(x_{n}\right)\right) \in S$ for all $n \in \mathbb{N}$. We may assume without loss of generality that $\pi\left(x_{n}, \tau_{S}\left(x_{n}\right)\right) \stackrel{n \rightarrow \infty}{\longrightarrow} y \in S$ as $S$ is compact.

If $\left\{\tau_{S}\left(x_{n}\right)\right\}_{n \in \mathbb{N}}$ admits a convergent subsequence, which we denote by the same, i.e., $\tau_{S}\left(x_{n}\right) \stackrel{n \rightarrow \infty}{\longrightarrow} r$, then

$$
\pi\left(x_{n}, \tau_{S}\left(x_{n}\right)\right) \stackrel{n \rightarrow \infty}{\longrightarrow} \pi(x, r)=y \in S,
$$

consequently, as $S$ is a section, $r=\tau_{S}(x)$, which contradicts our assumption.

Also, if $\left\{\tau_{S}\left(x_{n}\right)\right\}_{n \in \mathbb{N}}$ admits an unbounded subsequence, which we denote by the same, with $\tau_{S}\left(x_{n}\right) \stackrel{n \rightarrow \infty}{\longrightarrow} \infty$, then $y \in J^{+}(x)$. But this contradicts the fact that $\left(X, \pi, \mathbb{R}_{+}\right)$is dispersive.

Case 2: $\left\{x_{n}\right\}_{n \in \mathbb{N}} \subset \pi\left(S, \mathbb{R}_{+}\right)$.

In this case, for each $n \in \mathbb{N}$, there is $z_{n} \in S$ such that $\pi\left(z_{n}, \tau_{S}\left(x_{n}\right)\right)=x_{n}$. Since $S$ is compact, we may assume that $z_{n} \stackrel{n \rightarrow \infty}{\longrightarrow} z \in S$. Now, proceeding as in the proof of Case 1, we get a contradiction.

Hence, $\tau_{S}$ is continuous on $X$ and $\left(X, \pi, \mathbb{R}_{+}\right)$is parallelizable.

Corollary 3.3.1. Let $\left(X, \pi, \mathbb{R}_{+}\right)$be dispersive. If $\left(X, \pi, \mathbb{R}_{+}\right)$is completely continuous and admits a bounded section $S$, then $\left(X, \pi, \mathbb{R}_{+}\right)$is parallelizable.

\subsection{Parallelizability versus negative semisolutions}

The theory of negative semisolutions of semidynamical systems was presented in Subsection 2.3.1, Chapter 2. In this section, we shall consider the following conditions:

H1) Any negative semisolution through $x \in X$ is defined on $I_{x}=(-\infty, 0]$. 
H2) Let $x \in X$ and $\left\{x_{n}\right\}_{n \in \mathbb{N}} \subset X$ be a sequence such that $x_{n} \stackrel{n \rightarrow \infty}{\longrightarrow} x$. Given a global negative semisolution $\sigma_{x}$ through $x$, there is a global negative semisolution $\sigma_{n}$ through $x_{n}$ such that $\sigma_{n}(t) \stackrel{n \rightarrow \infty}{\longrightarrow} \sigma_{x}(t)$ for all $t \leqslant 0$.

Definition 3.4.1. A semidynamical system $\left(X, \pi, \mathbb{R}_{+}\right)$has the property of negative unicity on a set $\mathscr{U} \subset X$, if for every $x \in \mathscr{U}$ and $t \in \mathbb{R}_{+}$such that $F(x, t) \subset \mathscr{U}$ then $F(x, t)$ is a singleton.

Definition 3.4.1 says that if $x \in \mathscr{U}$ and $\sigma_{x}^{1}, \sigma_{x}^{2}$ are negative semisolutions through $x$, then $\sigma_{x}^{1}(t)=\sigma_{x}^{2}(t)$ for all $t \in I_{x}$ whereas $\sigma_{x}^{1}(t), \sigma_{x}^{2}(t) \subset \mathscr{U}$.

Instead of compactness of sections (see Theorem 3.2.2), we also can obtain the converse of Theorem 3.2.1 using the existence of global negative semisolutions.

Theorem 3.4.1. Assume that $\left(X, \pi, \mathbb{R}_{+}\right)$admits a section $S$ with a continuous section function $\tau_{S}$. Under the conditions $H 1)$ and $H 2)$, assume that $\left(X, \pi, \mathbb{R}_{+}\right)$has the property of negative unicity in $\pi\left(S, \mathbb{R}_{+}\right)$. Then the system $\left(X, \pi, \mathbb{R}_{+}\right)$is parallelizable.

Proof. Given $x \in \pi\left(S, \mathbb{R}_{+}\right)$, let $\sigma_{x}$ be a global negative semisolution through $x$. Define the map $h: X \rightarrow S \times \mathbb{R}_{+}$by

$$
h(x)=\left\{\begin{array}{lll}
\left(\pi\left(x, \tau_{S}(x)\right), \tau_{S}(x)\right) & \text { if } & x \in F\left(S, \mathbb{R}_{+}\right), \\
\left(\sigma_{x}\left(-\tau_{S}(x)\right), \tau_{S}(x)\right) & \text { if } & x \in \pi\left(S, \mathbb{R}_{+}\right) .
\end{array}\right.
$$

Let $z \in S$ and $t \geqslant 0$. At first note that, since $\sigma_{\pi(z, t)}$ is a negative semisolution through $\pi(z, t)$ and we have the negative unicity property on $\pi\left(S, \mathbb{R}_{+}\right)$, we get $\sigma_{\pi(z, t)}(-t)=z$. Thus,

$$
h(\pi(z, t))=\left(\sigma_{\pi(z, t)}\left(-\tau_{S}(\pi(z, t))\right), \tau_{S}(\pi(z, t))\right)=\left(\sigma_{\pi(z, t)}(-t), t\right)=(z, t) .
$$

Also, if $x \in F(z, t)$, then

$$
h(x)=\left(\pi\left(x, \tau_{S}(x)\right), \tau_{S}(x)\right)=(z, t) .
$$

Hence, the conditions $(i)$ and (ii) of Definition 3.1.2 hold.

It remains to prove that $h$ is continuous on $X$. Let $x \in X$ and $\left\{x_{n}\right\}_{n \in \mathbb{N}} \subset X$ be a sequence such that $x_{n} \stackrel{n \rightarrow \infty}{\longrightarrow} x$. If $x \in \pi(S,(0, \infty))$ or $x \in F(S,(0, \infty))$, then the proof is straightforward since $\pi(S,(0, \infty))$ and $F(S,(0, \infty))$ are open sets (see Lemma 3.1.3), $\pi$ and $\tau_{S}$ are continuous, and condition $\mathrm{H} 2$ ) holds. If $x \in S$, it is enough to note that

$$
\tau_{S}\left(x_{n}\right) \stackrel{n \rightarrow \infty}{\longrightarrow} 0 .
$$

Thus, $h\left(x_{n}\right) \stackrel{n \rightarrow \infty}{\longrightarrow} h(x)$. Therefore, the system $\left(X, \pi, \mathbb{R}_{+}\right)$is parallelizable. 
CHAPTER

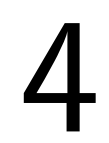

\section{PARALLELIZABLE IMPULSIVE SEMIDYNAMICAL SYSTEMS}

This chapter devotes the study of parallelizable impulsive semidynamical systems. As discussed in Subsection 2.2.1 from Chapter 2, the continuity of the impact function $\phi$ plays an important role to assure the well behavior of impulsive trajectories. However, it is not easy to obtain tubes through points from $M$ in many applications (specially in infinite dimensional spaces). Thus, in Section 4.1, we establish a new condition that assures the continuity of $\phi$ which is less complicated compared to tube conditions. In Sections 4.2, 4.3 and 4.4 we present the results concerning parallelizability.

The results of this chapter are presented in the paper (BONOTTO; PACIFICO, 2021a).

\subsection{Continuity of the impact function $\phi$}

In Definition 4.1.1, we introduce the condition (C). As we will see in the sequel, this condition will assure the continuity of $\phi$ outside the impulsive set $M$.

Definition 4.1.1. We say that a point $x \in M$ satisfies the condition (C) if for every sequence $\left\{x_{n}\right\}_{n \in \mathbb{N}} \subset X$ such that $x_{n} \stackrel{n \rightarrow \infty}{\longrightarrow} x$, then one can find a subsequence $\left\{x_{n_{k}}\right\}_{k \in \mathbb{N}}$ of $\left\{x_{n}\right\}_{n \in \mathbb{N}}$ such that

(C1) either $\phi\left(x_{n_{k}}\right) \stackrel{k \rightarrow \infty}{\longrightarrow} 0$, 
(C2) or there exists a sequence of non-negative numbers $\gamma_{k} \stackrel{k \rightarrow \infty}{\longrightarrow} 0$ such that $F\left(x_{n_{k}}, \gamma_{k}\right) \subset M$ for all $k \in \mathbb{N}$ with $\liminf _{k \rightarrow \infty}\left[\phi\left(x_{n_{k}}\right)-\gamma_{k}\right]>0$.

The set $M$ satisfies the condition (C) if each point of $M$ satisfies the condition (C).

Example 4.1.1. In Example 2.2.3, the point $(0,0)$ does not satisfy condition (C) since $\phi\left(0, \frac{1}{n}\right)=\infty$ for every $n \in \mathbb{N}$.

Example 4.1.2. Let $\left(\mathbb{R}^{2}, \pi, M, I\right)$ be an ISS, where $M=\left\{(x, y): x^{2}+y^{2}=1\right\}, I: M \rightarrow \mathbb{R}^{2}$ is a continuous function and

$$
\pi((x, y), t)=\left(x e^{-t}, y e^{-t}\right),
$$

for all $(x, y) \in \mathbb{R}^{2}$ and all $t \geqslant 0$. Every point from $M$ satisfies condition (C).

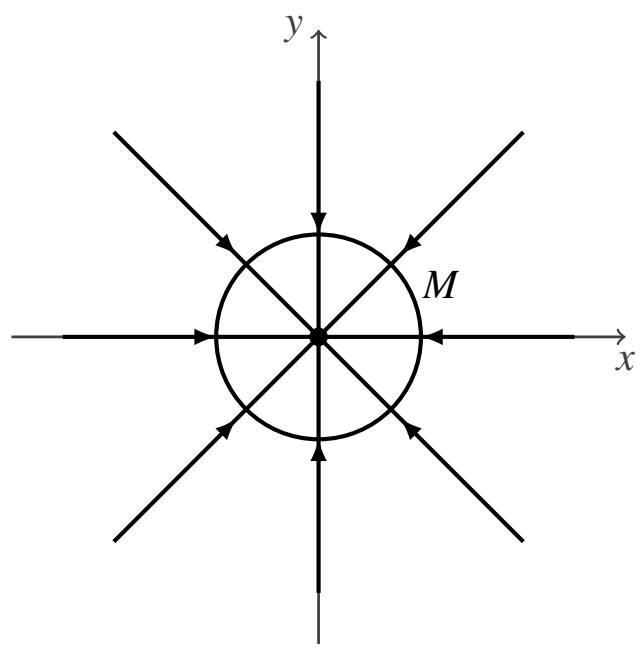

Figure 4.1: Positive trajectories of the semidynamical system (4.1.1).

The next result shows that the strong tube condition implies the condition (C).

Lemma 4.1.1. Consider an ISS $(X, \pi, M, I)$ and assume that $x \in M$ satisfies STC. Then $x$ satisfies the condition $(\mathrm{C})$.

Proof. Since $x$ satisfies STC, there exists a STC $\lambda$-tube $F(L,[0,2 \lambda])$ through $x$ with $\lambda$-section $S$. As the $\lambda$-tube is a neighborhood of $x$, there is $\eta>0$ such that $B(x, \eta) \subset F(L,[0,2 \lambda])$. Denote by $H_{1}$ and $H_{2}$ the following sets

$$
H_{1}=F(L,(\lambda, 2 \lambda]) \bigcap B(x, \eta) \text { and } H_{2}=F(L,[0, \lambda]) \bigcap B(x, \eta),
$$


as shown in Figure 4.2.

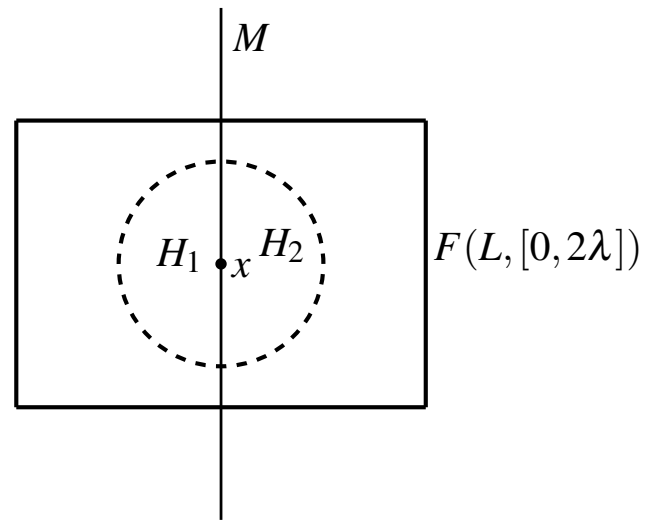

Figure 4.2: $H_{1}=F(L,(\lambda, 2 \lambda]) \bigcap B(x, \eta) \quad$ and $\quad H_{2}=F(L,[0, \lambda]) \bigcap B(x, \eta)$.

Let $\left\{x_{n}\right\}_{n \in \mathbb{N}}$ be a sequence such that $x_{n} \stackrel{n \rightarrow \infty}{\longrightarrow} x$. Then $\left\{x_{n}\right\}_{n \in \mathbb{N}}$ admits a subsequence $\left\{x_{n_{k}}\right\}_{k \in \mathbb{N}}$ such that either $\left\{x_{n_{k}}\right\}_{k \in \mathbb{N}} \subset H_{1}$ or $\left\{x_{n_{k}}\right\}_{k \in \mathbb{N}} \subset H_{2}$.

Let us consider the case $\left\{x_{n_{k}}\right\}_{k \in \mathbb{N}} \subset H_{1}$. For each $k \in \mathbb{N}$, there exists $\alpha_{k} \in(\lambda, 2 \lambda]$ such that $\pi\left(x_{n_{k}}, \alpha_{k}\right) \in L$. Note that

$$
\pi\left(x_{n_{k}}, \alpha_{k}-\lambda\right) \in S, \quad k \in \mathbb{N},
$$

since $\pi\left(x_{n_{k}}, \alpha_{k}\right) \in L$ and $F(L, \lambda)=S$ according to Definition 2.2.13. On the other hand, we have $S \subset M$ as the $\lambda$-tube satisfies STC, consequently,

$$
\phi\left(x_{n_{k}}\right) \leqslant \alpha_{k}-\lambda, \quad \text { for all } k \in \mathbb{N} .
$$

We may assume, up to a subsequence, that $\alpha_{k} \stackrel{k \rightarrow \infty}{\longrightarrow} \bar{\alpha} \in[\lambda, 2 \lambda]$. We claim that $\bar{\alpha}=\lambda$. Indeed, when $k \rightarrow \infty$ in (4.1.2), we obtain using the closeness of $S$

$$
\pi(x, \bar{\alpha}-\lambda) \in S=F(L, \lambda),
$$

that is, $\pi(x, \bar{\alpha}) \in L$. But since $x \in S$, it follows by the property of tube that $\bar{\alpha}=\lambda$. This proves the claim. Lastly, as $k \rightarrow \infty$ in (4.1.3), we obtain $\phi\left(x_{n_{k}}\right) \stackrel{k \rightarrow \infty}{\longrightarrow} 0$.

Now, let us consider the case $\left\{x_{n_{k}}\right\}_{k \in \mathbb{N}} \subset H_{2}$. Here, there exists $\beta_{k} \in[0, \lambda]$ such that $\pi\left(x_{n_{k}}, \beta_{k}\right) \in L$ for all $k \in \mathbb{N}$. We may assume, up to a subsequence, that $\beta_{k} \stackrel{k \rightarrow \infty}{\longrightarrow} \bar{\beta} \in[0, \lambda]$. We claim that $\bar{\beta}=\lambda$. In fact, since $L$ is closed we get $\pi(x, \bar{\beta}) \in L$. Again, by the property of tube, we conclude that $\bar{\beta}=\lambda$ which shows the claim. Now, define the sequence $\gamma_{k}=\lambda-\beta_{k}, k \in \mathbb{N}$. 
Note that $\gamma_{k} \stackrel{k \rightarrow \infty}{\longrightarrow} 0$ and $F\left(x_{n_{k}}, \gamma_{k}\right) \subset M$ for all $k \in \mathbb{N}$ since $\pi\left(F\left(x_{n_{k}}, \gamma_{k}\right), \lambda\right) \subset L$. Furthermore, since $S=F(L,[0,2 \lambda]) \cap M$, we have $\liminf _{k \rightarrow \infty}\left[\phi\left(x_{n_{k}}\right)-\gamma_{k}\right] \geqslant \lambda>0$.

In conclusion, $x$ satisfies the condition (C).

In Theorem 4.1.1, we establish the continuity of $\phi$ on $X \backslash M$ by using the condition (C). Before that, we exhibit an auxiliary result.

Lemma 4.1.2. Consider an ISS $(X, \pi, M, I)$. Assume that each element of $M$ satisfies the condition (C). If $x \notin M, \phi(x)<\infty$ and $x_{n} \stackrel{n \rightarrow \infty}{\longrightarrow} x$ then $\left\{\phi\left(x_{n}\right)\right\}_{n \in \mathbb{N}}$ is bounded.

Proof. Suppose to the contrary that $\left\{\phi\left(x_{n}\right)\right\}_{n \in \mathbb{N}}$ admits a subsequence $\left\{\phi\left(x_{n_{k}}\right)\right\}_{k \in \mathbb{N}}$ such that $\phi\left(x_{n_{k}}\right) \stackrel{k \rightarrow \infty}{\longrightarrow} \infty$. Since $\pi\left(x_{n_{k}}, \phi(x)\right) \stackrel{k \rightarrow \infty}{\longrightarrow} \pi(x, \phi(x)) \in M$, it follows by condition (C) that one can find a subsequence, which we denote by the same, such that

(A) either $\phi\left(\pi\left(x_{n_{k}}, \phi(x)\right)\right) \stackrel{k \rightarrow \infty}{\longrightarrow} 0$;

(B) or there exists a sequence of non-negative numbers $\gamma_{k} \stackrel{k \rightarrow \infty}{\longrightarrow} 0$ such that

$$
F\left(\pi\left(x_{n_{k}}, \phi(x)\right), \gamma_{k}\right) \subset M
$$

for all $k \in \mathbb{N}$ with $\liminf _{k \rightarrow \infty}\left[\phi\left(\pi\left(x_{n_{k}}, \phi(x)\right)\right)-\gamma_{k}\right]>0$.

If condition (A) holds, then by using the fact that

$$
\pi\left(\pi\left(x_{n_{k}}, \phi(x)\right), \phi\left(\pi\left(x_{n_{k}}, \phi(x)\right)\right)\right) \in M, \quad k \in \mathbb{N},
$$

we get $\phi\left(x_{n_{k}}\right) \leqslant \phi(x)+\phi\left(\pi\left(x_{n_{k}}, \phi(x)\right)\right)$ for all $k \in \mathbb{N}$ and it is a contradiction.

If condition (B) holds, then since $\pi\left(x_{n_{k}}, \phi(x)-\gamma_{k}\right) \in F\left(\pi\left(x_{n_{k}}, \phi(x)\right), \gamma_{k}\right) \subset M$ for $k$ sufficiently large, we have

$$
\phi\left(x_{n_{k}}\right) \leqslant \phi(x)-\gamma_{k}
$$

for $k$ sufficiently large, which leads us again to a contradiction.

Therefore, $\left\{\phi\left(x_{n}\right)\right\}_{n \in \mathbb{N}}$ is bounded.

Theorem 4.1.1. Consider an ISS $(X, \pi, M, I)$. Assume that each element of $M$ satisfies the condition (C). Then $\phi$ is continuous at $x$ if and only if $x \in X \backslash M$. 
Proof. Assume that $\phi$ is continuous at $x \in X$. If $x \in M$ then it follows by condition (2.2.1) that there exists $\varepsilon_{x}>0$ such that $F\left(x,\left(0, \varepsilon_{x}\right)\right) \bigcap M=\emptyset$. Let $y \in F\left(x,\left(0, \varepsilon_{x}\right)\right)$ and $s \in\left(0, \varepsilon_{x}\right)$ be such that $\pi(y, s)=x$. Let $\left\{\beta_{n}\right\}_{n \in \mathbb{N}} \subset(0, s)$ be a sequence such that $\beta_{n} \stackrel{n \rightarrow \infty}{\longrightarrow} 0$. Then, defining $x_{n}=$ $\pi\left(y, s-\beta_{n}\right), n \in \mathbb{N}$, we have

$$
x_{n} \stackrel{n \rightarrow \infty}{\longrightarrow} x \quad \text { and } \quad \phi\left(x_{n}\right)=\beta_{n} \stackrel{n \rightarrow \infty}{\longrightarrow} 0<\phi(x),
$$

which is a contradiction. Hence, $x \notin M$.

Now, let $x \in X \backslash M$ and $\left\{x_{n}\right\}_{n \in \mathbb{N}} \subset X$ be a sequence such that $x_{n} \stackrel{n \rightarrow \infty}{\longrightarrow} x$. We may assume that $\left\{x_{n}\right\}_{n \in \mathbb{N}} \subset X \backslash M$ since $M$ is a closed subset of $X$. Furthermore, we may assume that $\phi(x)<\infty$. By Lemma 4.1.2, $\left\{\phi\left(x_{n}\right)\right\}_{n \in \mathbb{N}}$ is bounded. Now, suppose to the contrary that $\left\{\phi\left(x_{n}\right)\right\}_{n \in \mathbb{N}}$ admits a subsequence $\left\{\phi\left(x_{n_{k}}\right)\right\}_{k \in \mathbb{N}}$ such that

$$
\phi\left(x_{n_{k}}\right) \stackrel{k \rightarrow \infty}{\longrightarrow} t_{0} \neq \phi(x)
$$

Since $\pi\left(x_{n_{k}}, \phi\left(x_{n_{k}}\right)\right) \stackrel{k \rightarrow \infty}{\longrightarrow} \pi\left(x, t_{0}\right)$ and $\left\{\pi\left(x_{n_{k}}, \phi\left(x_{n_{k}}\right)\right)\right\}_{k \in \mathbb{N}} \subset M$, we get $\pi\left(x, t_{0}\right) \in M$. Thus,

$$
\phi(x)<t_{0}
$$

since $\phi(x) \neq t_{0}$.

On the other hand, since $\pi\left(x_{n_{k}}, \phi(x)\right) \stackrel{k \rightarrow \infty}{\longrightarrow} \pi(x, \phi(x)) \in M$, it follows by condition (C) that one can find a subsequence, which we denote by the same, such that either $\phi\left(\pi\left(x_{n_{k}}, \phi(x)\right)\right) \stackrel{k \rightarrow \infty}{\longrightarrow} 0$ or there exists a sequence of non-negative numbers $\gamma_{k} \stackrel{k \rightarrow \infty}{\longrightarrow} 0$ such that $F\left(\pi\left(x_{n_{k}}, \phi(x)\right), \gamma_{k}\right) \subset M$ for all $k \in \mathbb{N}$ with $\liminf _{k \rightarrow \infty}\left[\phi\left(\pi\left(x_{n_{k}}, \phi(x)\right)\right)-\gamma_{k}\right]>0$.

If $\phi\left(\pi\left(x_{n_{k}}, \phi(x)\right)\right) \stackrel{n \rightarrow \infty}{\longrightarrow} 0$ then by the proof of Lemma 4.1 .2 we obtain $\phi\left(x_{n_{k}}\right) \leqslant \phi(x)+$ $\phi\left(\pi\left(x_{n_{k}}, \phi(x)\right)\right)$ for all $k \in \mathbb{N}$. As $k \rightarrow \infty$, we get $t_{0} \leqslant \phi(x)$ which contradicts (4.1.4).

If there exists a sequence $\gamma_{k} \stackrel{k \rightarrow \infty}{\longrightarrow} 0$ such that $F\left(\pi\left(x_{n_{k}}, \phi(x)\right), \gamma_{k}\right) \subset M$ for all $k \in \mathbb{N}$, then by the proof of Lemma 4.1.2 we obtain $t_{0}=\lim _{k \rightarrow \infty} \phi\left(x_{n_{k}}\right) \leqslant \lim _{k \rightarrow \infty}\left(\phi(x)-\gamma_{k}\right)=\phi(x)$ and it contradicts (4.1.4).

Hence, $\phi\left(x_{n}\right) \stackrel{n \rightarrow \infty}{\longrightarrow} \phi(x)$ and $\phi$ is continuous on $X \backslash M$.

Corollary 4.1.1. Let $(X, \pi, M, I)$ be an ISS such that each element of $M$ satisfies the condition (C). Let $x \in M$ and $\left\{x_{n}\right\}_{n \in \mathbb{N}} \subset X$ be a sequence such that $x_{n} \stackrel{n \rightarrow \infty}{\longrightarrow} x$. Then there is a subsequence $\left\{x_{n_{k}}\right\}_{k \in \mathbb{N}}$ of $\left\{x_{n}\right\}_{n \in \mathbb{N}}$ such that either $\phi\left(x_{n_{k}}\right) \stackrel{k \rightarrow \infty}{\longrightarrow} 0$ or $\phi\left(x_{n_{k}}\right) \stackrel{k \rightarrow \infty}{\longrightarrow} \phi(x)$. 
Proof. According to condition (C), one can find a subsequence $\left\{x_{n_{k}}\right\}_{k \in \mathbb{N}}$ of $\left\{x_{n}\right\}_{n \in \mathbb{N}}$ such that either $\phi\left(x_{n_{k}}\right) \stackrel{k \rightarrow \infty}{\longrightarrow} 0$ or condition (C2) from Definition 4.1.1 holds. In this last case, there exists a sequence of non-negative numbers $\gamma_{k} \stackrel{k \rightarrow \infty}{\longrightarrow} 0$ such that $\liminf _{k \rightarrow \infty}\left[\phi\left(x_{n_{k}}\right)-\gamma_{k}\right]>0$. Since $x \in M$, it follows by (2.2.1) that there is $\varepsilon_{x}>0$ such that $\pi\left(x,\left(0, \varepsilon_{x}\right)\right) \cap M=\emptyset$. We claim that $\pi\left(x_{n_{k}},\left(0, \frac{\phi(x)}{2}\right)\right) \cap M=\emptyset$ for $k$ sufficiently large. Indeed, suppose to the contrary, up to a subsequence, that there exists $\lambda_{k} \in$ $\left(0, \frac{\phi(x)}{2}\right)$ such that $\pi\left(x_{n_{k}}, \lambda_{k}\right) \in M$ for every $k \in \mathbb{N}$. The sequence $\left\{\lambda_{k}\right\}_{k \in \mathbb{N}}$ admits a subsequence (which we denote by the same) such that $\lambda_{k} \stackrel{k \rightarrow \infty}{\longrightarrow} t_{0} \in\left[0, \frac{\phi(x)}{2}\right]$. Since $\liminf _{k \rightarrow \infty}\left[\phi\left(x_{n_{k}}\right)-\gamma_{k}\right]>0$, we have $t_{0} \neq 0$. Consequently,

$$
\pi\left(x_{n_{k}}, \lambda_{k}\right) \stackrel{k \rightarrow \infty}{\longrightarrow} \pi\left(x, t_{0}\right) \in M
$$

and it is a contradiction as $0<t_{0}<\phi(x)$. Hence, the claim is verified. In this way, for $0<s_{0}<\frac{\phi(x)}{2}$, we have

$$
\tilde{\pi}\left(x_{n_{k}}, s_{0}\right)=\pi\left(x_{n_{k}}, s_{0}\right) \stackrel{k \rightarrow \infty}{\longrightarrow} \pi\left(x, s_{0}\right)=\tilde{\pi}\left(x, s_{0}\right) \notin M .
$$

By Theorem 4.1.1, $\phi\left(\tilde{\pi}\left(x_{n_{k}}, s_{0}\right)\right) \stackrel{k \rightarrow \infty}{\longrightarrow} \phi\left(\tilde{\pi}\left(x, s_{0}\right)\right)$ which implies

$$
\phi\left(x_{n_{k}}\right)=s_{0}+\phi\left(\tilde{\pi}\left(x_{n_{k}}, s_{0}\right)\right) \stackrel{k \rightarrow \infty}{\longrightarrow} s_{0}+\phi\left(\tilde{\pi}\left(x, s_{0}\right)\right)=\phi(x)
$$

and this concludes the proof.

In what follows, we establish some convergence results. The condition (C) guarantees the continuity of $\phi$ on $X \backslash M$.

Lemma 4.1.3. Let $(X, \pi, M, I)$ be an ISS such that $I(M) \bigcap M=\varnothing$ and each point of $M$ satisfies condition (C). Let also $x \in X \backslash M$ and $\left\{x_{n}\right\}_{n \in \mathbb{N}}$ be a sequence in $X$ such that $x_{n} \stackrel{n \rightarrow \infty}{\longrightarrow} x$. Then, given $t \geqslant 0$, there exists a sequence $\left\{\eta_{n}\right\}_{n \in \mathbb{N}}$ in $\mathbb{R}$ such that $\eta_{n} \stackrel{n \rightarrow \infty}{\longrightarrow} 0$ and $\tilde{\pi}\left(x_{n}, t+\eta_{n}\right) \stackrel{n \rightarrow \infty}{\longrightarrow} \tilde{\pi}(x, t)$.

Proof. We may use the proof of (BONOTTO et al., 2015, Lemma 3.6) as $\phi$ is continuous on $X \backslash M$.

Remark 4.1.1. Under the assumptions of Lemma 4.1.3, assume that $t$ is a jump time of $x$ (see Definition 2.2.7). By the proof of (BONOTTO et al., 2015, Corollary 3.6), we may conclude that there exists a sequence $\left\{\eta_{n}\right\}_{n \in \mathbb{N}}$ in $\mathbb{R}$ such that $\eta_{n} \stackrel{n \rightarrow \infty}{\longrightarrow} 0, \tilde{\pi}\left(x_{n}, t+\eta_{n}\right) \in I(M)$ and $\tilde{\pi}\left(x_{n}, t+\eta_{n}\right) \stackrel{n \rightarrow \infty}{\longrightarrow}$ $\tilde{\pi}(x, t)$. In this case, we may conclude that $\tilde{\pi}\left(x_{n}, t+\eta_{n}\right)$ belongs to the set $I(M)$ for every $n \in \mathbb{N}$.

Remark 4.1.2. Under the assumptions of Lemma 4.1.3, assume that $t$ is a non-jump time of $x$, i.e., $t \neq \sum_{j=0}^{k-1} \phi\left(x_{j}^{+}\right)$for all $k \in \mathbb{N}$. By the proof of (BONOTTO et al., 2015, Corollary 3.6), we may conclude that $\tilde{\pi}\left(x_{n}, t\right) \stackrel{n \rightarrow \infty}{\longrightarrow} \tilde{\pi}(x, t)$. Here, the correction time $\eta_{n}$ is not necessary. 
Lemma 4.1.4. Let $(X, \pi, M, I)$ be an ISS such that each point in $M$ satisfies condition (C), $x \notin M$ and $\left\{x_{n}\right\}_{n \in \mathbb{N}}$ be a sequence in $X \backslash M$ such that $x_{n} \stackrel{n \rightarrow \infty}{\longrightarrow} x$. Then if $\alpha_{n} \stackrel{n \rightarrow \infty}{\longrightarrow} 0$ and $\alpha_{n} \geqslant 0$, for all $n \in \mathbb{N}$, we have $\tilde{\pi}\left(x_{n}, \alpha_{n}\right) \stackrel{n \rightarrow \infty}{\longrightarrow} x$.

Proof. We may use the proof of (BONOTTO et al., 2015, Lemma 3.8) as $\phi$ is continuous on $X \backslash M$.

Corollary 4.1.2. Under the assumptions of Lemma 4.1.3, there exists a sequence $\left\{\varepsilon_{n}\right\}_{n \in \mathbb{N}} \subseteq[0, \infty)$ such that $\varepsilon_{n} \stackrel{n \rightarrow \infty}{\longrightarrow} 0$ and $\tilde{\pi}\left(x_{n}, t+\varepsilon_{n}\right) \stackrel{n \rightarrow \infty}{\longrightarrow} \tilde{\pi}(x, t)$.

Proof. We may use the proof of (BONOTTO et al., 2015, Corollary 3.9) as $\phi$ is continuous on $X \backslash M$.

Lemma 4.1.5. Let $(X, \pi, M, I)$ be an ISS such that $I(M) \cap M=\varnothing$ and each point of $M$ satisfies condition (C). Let also $x \in X \backslash M, t \in \mathbb{R}_{+},\left\{x_{n}\right\}_{n \in \mathbb{N}} \subset X$ and $\left\{t_{n}\right\}_{n \in \mathbb{N}} \subset \mathbb{R}_{+}$be sequences such that $x_{n} \stackrel{n \rightarrow \infty}{\longrightarrow} x$ and $t_{n} \stackrel{n \rightarrow \infty}{\longrightarrow} t$. Then there exists a sequence $\left\{\beta_{n}\right\}_{n \in \mathbb{N}}$ in $\mathbb{R}_{+}$such that $\beta_{n} \stackrel{n \rightarrow \infty}{\longrightarrow} 0$ and $\tilde{\pi}\left(x_{n}, t_{n}+\beta_{n}\right) \stackrel{n \rightarrow \infty}{\longrightarrow} \tilde{\pi}(x, t)$. In addition, if $t \in \mathbb{R}_{+}$is a non-jump time of $x$ then $\tilde{\pi}\left(x_{n}, t_{n}\right) \stackrel{n \rightarrow \infty}{\longrightarrow} \tilde{\pi}(x, t)$.

Proof. By Lemma 4.1.3 and Corollary 4.1.2, there exists a sequence $\left\{\varepsilon_{n}\right\}_{n \in \mathbb{N}} \subseteq[0, \infty)$ such that $\varepsilon_{n} \stackrel{n \rightarrow \infty}{\longrightarrow} 0$ and $\tilde{\pi}\left(x_{n}, t+\varepsilon_{n}\right) \stackrel{n \rightarrow \infty}{\longrightarrow} \tilde{\pi}(x, t)$. Using Lemma 4.1.4, with $\alpha_{n}=t_{n}-t+\left|t_{n}-t\right|, n \in \mathbb{N}$, we get $\tilde{\pi}\left(x_{n}, t_{n}+\left|t_{n}-t\right|+\varepsilon_{n}\right) \stackrel{n \rightarrow \infty}{\longrightarrow} \tilde{\pi}(x, t)$. Hence, it is enough to take $\beta_{n}=\varepsilon_{n}+\left|t_{n}-t\right|, n \in \mathbb{N}$.

Now, assume that $t$ is a non-jump time of $x$. If $\phi(x)=\infty$ then the result is immediate. If there exists $m \in \mathbb{N}$ such that $\phi\left(x_{m}^{+}\right)=\infty, \phi\left(x_{m-1}^{+}\right)<\infty$ and $t>\sum_{j=0}^{m-1} \phi\left(x_{j}^{+}\right)$then, by using the continuity of $\phi$ on $X \backslash M$ and the continuity of $I$ on $M$, we obtain $\phi\left(\left(x_{n}\right)_{m}^{+}\right)=\infty, \phi\left(\left(x_{n}\right)_{m-1}^{+}\right)<\infty$ and $t_{n}>\sum_{j=0}^{m-1} \phi\left(\left(x_{n}\right)_{j}^{+}\right)$for $n$ sufficiently large. This brings us to the convergence

$$
\tilde{\pi}\left(x_{n}, t_{n}\right)=\pi\left(\left(x_{n}\right)_{m}^{+}, t_{n}\right) \stackrel{n \rightarrow \infty}{\longrightarrow} \pi\left(x_{m}^{+}, t\right)=\tilde{\pi}(x, t) .
$$

On the other hand, if $t=\sum_{j=0}^{k-1} \phi\left(x_{j}^{+}\right)+t^{\prime}$ for some $k \in \mathbb{N}$ with $0<t^{\prime}<\phi\left(x_{k}^{+}\right)<\infty$, then we conclude that there exist $n_{0} \in \mathbb{N}$ and $t_{n}^{\prime} \in\left(0, \phi\left(\left(x_{n}\right)_{k}^{+}\right)\right)$for $n \geqslant n_{0}$, such that $t_{n}^{\prime} \stackrel{n \rightarrow \infty}{\longrightarrow} t^{\prime}$ and

$$
t_{n}=\sum_{j=0}^{k-1} \phi\left(\left(x_{n}\right)_{j}^{+}\right)+t_{n}^{\prime}, \quad n \geqslant n_{0},
$$

because $\sum_{j=0}^{k-1} \phi\left(\left(x_{n}\right)_{j}^{+}\right) \stackrel{n \rightarrow \infty}{\longrightarrow} \sum_{j=0}^{k-1} \phi\left(x_{j}^{+}\right)$and $t_{n} \stackrel{n \rightarrow \infty}{\longrightarrow} t$. Consequently,

$$
\tilde{\pi}\left(x_{n}, t_{n}\right)=\pi\left(\left(x_{n}\right)_{k}^{+}, t_{n}^{\prime}\right) \stackrel{n \rightarrow \infty}{\longrightarrow} \pi\left(x_{k}^{+}, t^{\prime}\right)=\tilde{\pi}(x, t) .
$$




\subsection{Parallelizable impulsive semidynamical systems}

In this section, we introduce the concepts of "parallelizability" and "section" in the context of impulsive semidynamical systems.

As presented in Chapter 3, in order to describe a parallelizable semidynamical system (without impulses), we need a base set $S \subset X$ and a structure to split $X$ in two component, namely, the past of $S$ described by the attainable set $F\left(S, \mathbb{R}_{+}\right)$and the forward of $S$ described by $\pi\left(S, \mathbb{R}_{+}\right)$. To generalize the concept of parallelizability in impulsive systems, we shall consider the following attainable impulsive set of $x \in X$ and $t \in \mathbb{R}_{+}$

$$
\tilde{F}(x, t)=\{y \in X: \tilde{\pi}(y, t)=x\}
$$

Note that if $I(M) \cap M=\emptyset$ and $x \in M$ then $\tilde{F}(x, t)=\emptyset$ for all $t>0$. Thus, every point in $M$ is an initial point in $(X, \pi, M, I)$ provided $I(M) \cap M=\emptyset$.

Definition 4.2.1. A subset $S \subset X$ is called a base for the ISS $(X, \pi, M, I)$ if $\bar{S} \subset X \backslash M$ and

$$
X=\tilde{F}\left(S, \mathbb{R}_{+}\right) \bigcup \tilde{\pi}\left(S, \mathbb{R}_{+}\right)
$$

Next, we establish the concept of parallelizability in impulsive semidynamical systems.

Definition 4.2.2. An ISS $(X, \pi, M, I)$ is said to be parallelizable, if there exist a base $S$ and a function $h: X \rightarrow S \times \mathbb{R}_{+}$satisfying the following conditions:

(i) $h$ is continuous on $X \backslash M$;

(ii) $h(\tilde{\pi}(x, t))=(x, t)$ for all $x \in S$ and $t \in \mathbb{R}_{+}$;

(iii) $h(\tilde{F}(x, t))=(x, t)$ for all $x \in S$ and $t \in \mathbb{R}_{+}$.

Example 4.2.1. Let $\left(\mathbb{R}^{2}, \pi, M, I\right)$ be an ISS, where $M=\{0\} \times \mathbb{R}, I(0, y)=(1, y)$ and $\pi((x, y), t)=$ $(x+t, y), x, y \in \mathbb{R}$ and $t \geqslant 0$. Note that $\left(\mathbb{R}^{2}, \pi, M, I\right)$ is parallelizable with base $S=\{1\} \times \mathbb{R}$ and function $h: \mathbb{R}^{2} \rightarrow S \times \mathbb{R}_{+}$given by

$$
h(a, b)= \begin{cases}((1, b),|a-1|) & \text { if } \quad a \geqslant 0 \\ ((1, b),|a|) & \text { if } \quad a<0\end{cases}
$$


According to Definition 4.2.2, a parallelizable ISS depends on a base $S$ and a function $h$. As shown in the next result, for a given base $S$ there exists a unique function $h$ satisfying conditions (i), (ii) and (iii) from the Definition 4.2.2.

Lemma 4.2.1. If $(X, \pi, M, I)$ is a parallelizable ISS with base $S$ then there exists a unique function $h: X \rightarrow S \times \mathbb{R}_{+}$satisfying conditions $(i),(i i)$ and (iii) of the Definition 4.2.2.

Proof. Suppose $h_{1}, h_{2}: X \rightarrow S \times \mathbb{R}_{+}$are functions which satisfy conditions $(i)$, (ii) and (iii) of Definition 4.2.2. Since $S$ is a base we have $\bar{S} \subset X \backslash M$ and $X=\tilde{F}\left(S, \mathbb{R}_{+}\right) \cup \tilde{\pi}\left(S, \mathbb{R}_{+}\right)$.

If $x \in \tilde{F}\left(S, \mathbb{R}_{+}\right)$, then there exist $y \in S$ and $t \in \mathbb{R}_{+}$such that $x \in \tilde{F}(y, t)$, consequently, due to condition $(i i i)$, we have $h_{1}(x)=(y, t)=h_{2}(x)$.

On the other hand, if $x \in \tilde{\pi}\left(S, \mathbb{R}_{+}\right)$then $x=\tilde{\pi}(z, t)$ for some $z \in S$ and $t \geqslant 0$. Thus, using condition $(i i)$, we get

$$
h_{1}(x)=h_{1}(\tilde{\pi}(z, t))=(z, t)=h_{2}(\tilde{\pi}(z, t))=h_{2}(x) .
$$

Hence, $h_{1}(x)=h_{2}(x)$ for all $x \in X$.

In the sequel, we present the version of Lemma 3.1.2 for the impulsive case.

Lemma 4.2.2. Let $(X, \pi, M, I)$ be parallelizable with base $S$. Then:

(a) $S$ is closed in $X$.

(b) $S, \tilde{\pi}(S,(0, \infty))$ and $\tilde{F}(S,(0, \infty))$ are pairwise disjoint sets.

(c) If $I(M) \bigcap M=\emptyset$ then $M \subset \tilde{F}(S,(0, \infty))$.

(d) If $M$ satisfies condition (C) and $I(M) \cap M=\emptyset$ then $\tilde{\pi}\left(S, \mathbb{R}_{+}\right)$and $\tilde{F}\left(S, \mathbb{R}_{+}\right)$are closed in $X$.

(e) If $M$ satisfies condition (C) and $I(M) \bigcap M=\emptyset$ then $\tilde{\pi}(S,(0, \infty))$ and $\tilde{F}(S,(0, \infty))$ are open in $X$.

Proof. Since $\left(X, \pi, \mathbb{R}_{+}\right)$is parallelizable, if follows by Definition 4.2.2 that there exists a function $h: X \rightarrow S \times \mathbb{R}_{+}$satisfying conditions $(i)$, (iii) and (iii).

(a) Note that $\bar{S} \cap M=\emptyset$ as $S$ is a base. Since $h^{-1}(S \times\{0\})=S, h$ is continuous on $X \backslash M$ and $S \times\{0\}$ is closed in $S \times \mathbb{R}_{+}$, we obtain $S$ is closed in $X$. 
(b) We are going to show that the sets $\tilde{\pi}(S,(0, \infty))$ and $\tilde{F}(S,(0, \infty))$ are pairwise disjoint since the other cases are analogous. Suppose to the contrary that there exists

$$
y \in \tilde{F}(S,(0, \infty)) \bigcap \tilde{\pi}(S,(0, \infty)) .
$$

Then there exist $z_{1}, z_{2} \in S$ and $t_{1}, t_{2} \in(0, \infty)$ with $\tilde{\pi}\left(z_{1}, t_{1}\right)=y$ and $\tilde{\pi}\left(y, t_{2}\right)=z_{2}$. Consequently,

$$
\left(z_{1}, t_{1}+t_{2}\right) \stackrel{(i i)}{=} h\left(\tilde{\pi}\left(z_{1}, t_{1}+t_{2}\right)\right)=h\left(\tilde{\pi}\left(y, t_{2}\right)\right)=h\left(z_{2}\right) \stackrel{(i i)}{=}\left(z_{2}, 0\right),
$$

which is a contradiction as $t_{1}, t_{2}>0$. Hence, $\tilde{F}(S,(0, \infty)) \cap \tilde{\pi}(S,(0, \infty))=\emptyset$.

(c) Suppose there is $y \in M \cap \tilde{\pi}(S,(0, \infty))$. Then there are $x \in S$ and $t>0$ such that $\tilde{\pi}(x, t)=$ $y \in M$. But this is a contradiction as $\tilde{F}(z, t)=\emptyset$ for all $z \in M$ and $t>0$ whenever $I(M) \bigcap M=\emptyset$. Consequently, $M \subset \tilde{F}(S,(0, \infty))$.

(d) Let $x \in \overline{\tilde{F}\left(S, \mathbb{R}_{+}\right)}$and $\left\{x_{n}\right\}_{n \in \mathbb{N}} \subset \tilde{F}\left(S, \mathbb{R}_{+}\right)$be a sequence such that $x_{n} \stackrel{n \rightarrow \infty}{\longrightarrow} x$. If $x \in M$ then $x \in \tilde{F}\left(S, \mathbb{R}_{+}\right)$since condition $(c)$ holds. Suppose $x \notin M$. By continuity of $h$ on $X \backslash M$ we have $h\left(x_{n}\right) \stackrel{n \rightarrow \infty}{\longrightarrow} h(x)$. Moreover, there is $(z, t) \in S \times \mathbb{R}_{+}$such that $h(x)=(z, t)$ as the range of $h$ is in $S \times \mathbb{R}_{+}$. On the other hand, for each $n \in \mathbb{N}$, there exist $z_{n} \in S$ and $t_{n} \in \mathbb{R}_{+}$such that $\tilde{\pi}\left(x_{n}, t_{n}\right)=z_{n}$. Using the properties of $h$ we obtain

$$
\left(z_{n}, t_{n}\right)=h\left(x_{n}\right) \stackrel{n \rightarrow \infty}{\longrightarrow} h(x)=(z, t) \in S \times \mathbb{R}_{+} .
$$

Using Lemma 4.1.5, one can find a sequence $\left\{\beta_{n}\right\}_{n \in \mathbb{N}}$ in $\mathbb{R}_{+}$such that $\beta_{n} \stackrel{n \rightarrow \infty}{\longrightarrow} 0$ and

$$
\tilde{\pi}\left(x_{n}, t_{n}+\beta_{n}\right) \stackrel{n \rightarrow \infty}{\longrightarrow} \tilde{\pi}(x, t) .
$$

Now, note that $\tilde{\pi}\left(x_{n}, t_{n}+\beta_{n}\right)=\tilde{\pi}\left(z_{n}, \beta_{n}\right), n \in \mathbb{N}$. But, by item $(c), \tilde{\pi}\left(S, \mathbb{R}_{+}\right)=\pi\left(S, \mathbb{R}_{+}\right)$. Thus,

$$
\tilde{\pi}\left(z_{n}, \beta_{n}\right)=\pi\left(z_{n}, \beta_{n}\right) \stackrel{n \rightarrow \infty}{\longrightarrow} z .
$$

Hence, $z=\tilde{\pi}(x, t)$. This shows that $x \in F\left(S, \mathbb{R}_{+}\right)$and $F\left(S, \mathbb{R}_{+}\right)$is closed in $X$.

Since $\tilde{\pi}\left(S, \mathbb{R}_{+}\right)=\pi\left(S, \mathbb{R}_{+}\right)$once $M \subset \tilde{F}(S,(0, \infty))$, we may apply the same proof of Lemma 3.1 .2 , item $(c)$, to conclude that $\tilde{\pi}\left(S, \mathbb{R}_{+}\right)$is closed in $X$.

(e) Since $\tilde{\pi}(S,(0, \infty))=X \backslash \tilde{F}\left(S, \mathbb{R}_{+}\right)$and $\tilde{F}(S,(0, \infty))=X \backslash \tilde{\pi}\left(S, \mathbb{R}_{+}\right)$, the result follows by item $(d)$.

Next, we present the notion of section for impulsive semidynamical systems. This definition is analogous to the definition of section in the context of continuous semidynamical systems, see Definition 3.1.3. 
Definition 4.2.3. A subset $S \subset X$ is called a section for an ISS $(X, \pi, M, I)$, if $\bar{S} \cap M=\emptyset$ and for each $x \in X$ either there is a unique $t_{x} \geqslant 0$ such that $\tilde{\pi}\left(x, t_{x}\right) \in S$ or there are unique $z_{x} \in S$ and $t_{x}>0$ such that $\tilde{\pi}\left(z_{x}, t_{x}\right)=x$.

Remark 4.2.1. Let $S \subset X$ be a section. Analogously as in Remark 3.1.1, if there exists a unique $t_{x}>0$ such that $\tilde{\pi}\left(x, t_{x}\right) \in S$ (thus, $\left.x \notin S\right)$, then $\tilde{F}\left(x, \mathbb{R}_{+}\right) \cap S=\emptyset$. In the case that $x \in S$, that is, $t_{x}=0$, it follows that $\tilde{F}(x,(0, \infty)) \cap S=\emptyset$ and $\tilde{\pi}(S,(0, \infty)) \bigcap S=\emptyset$. Also, if there are unique $z_{x} \in S$ and $t_{x}>0$ such that $\tilde{\pi}\left(z_{x}, t_{x}\right)=x$, then $\tilde{\pi}\left(x, \mathbb{R}_{+}\right) \cap S=\emptyset$.

Remark 4.2.1 implies, in particular, that we can decompose the phase space $X$ into

$$
X=A \cup S \cup B
$$

where

$$
A=\{w \in X \backslash S: \tilde{F}(w,(0, \infty)) \bigcap S \neq \emptyset\} \quad \text { and } \quad B=\{w \in X \backslash S: \tilde{\pi}(w,(0, \infty)) \bigcap S \neq \emptyset\}
$$

On the other hand, given a nonempty set $S \subset X$ with $\bar{S} \cap M=\emptyset$, if $X=A \cup S \cup B$, where $A$ and $B$ are given as before, and the uniqueness of $t_{x}$ holds (as stated in Definition 4.2.3 for each $x \in X$ ), then $S$ is a section.

Let $(X, \pi, M, I)$ be an ISS. Assume that $(X, \pi, M, I)$ admits a section $S$. Then we may define a mapping

$$
\tau_{S}: X \rightarrow \mathbb{R}_{+} \quad \text { by } \quad \tau_{S}(x)=t_{x}
$$

called section function, where $t_{x}$ comes from Definition 4.2.3. Note that $t_{x}>0$ if $x \notin S$ and $t_{x}=0$ provided $x \in S$. Thus, if $S$ is a section for $(X, \pi, M, I)$ then given $x \in X$,

$$
\text { either } \quad \tilde{\pi}\left(x, \tau_{S}(x)\right) \in S \quad \text { or } \quad \tilde{F}\left(x, \tau_{S}(x)\right)=\left\{z_{x}\right\} \subset S \text {. }
$$

Clearly, every section $S$ of $(X, \pi, M, I)$ is a base, i.e.,

$$
X=\tilde{F}\left(S, \mathbb{R}_{+}\right) \bigcup \tilde{\pi}\left(S, \mathbb{R}_{+}\right)=\tilde{F}(S,(0, \infty)) \bigcup S \bigcup \tilde{\pi}(S,(0, \infty))
$$

Besides that, the sets $S, \tilde{\pi}(S,(0, \infty))$ and $\tilde{F}(S,(0, \infty))$ are pairwise disjoint sets.

Lemma 4.2.3. If $S$ is a section for an ISS $(X, \pi, M, I)$ and $I(M) \bigcap M=\emptyset$, then $M \subset \tilde{F}(S,(0, \infty))$. Moreover, if $x \in \tilde{\pi}\left(S, \mathbb{R}_{+}\right)$then any time $t \in \mathbb{R}_{+}$is a non-jump time of $x$. 
Proof. The proof that $M \subset \tilde{F}(S,(0, \infty))$ is analogous to the proof of item $(c)$ of Lemma 4.2.2. Since $M \subset \tilde{F}(S,(0, \infty))$, it follows that $\tilde{\pi}\left(S, \mathbb{R}_{+}\right) \cap M=\emptyset$. Consequently, if $x \in \tilde{\pi}\left(S, \mathbb{R}_{+}\right)$then $\phi(x)=\infty$, that is, any time $t \in \mathbb{R}_{+}$is a non-jump time of $x$.

Lemma 4.2.4. Assume that $(X, \pi, M, I)$ admits a section $S$ with section function $\tau_{S}$ continuous on $X \backslash M$. Then $S$ is closed in $X$.

Proof. It is enough to note that $\bar{S} \bigcap M=\emptyset$ and $S=\tau_{S}^{-1}(\{0\})$.

Next, we exhibit an auxiliary lemma.

Lemma 4.2.5. Let $(X, \pi, M, I)$ be an ISS with section $S, x \in X$ and $t \geqslant 0$.

(a) If $x \in \tilde{F}\left(S, \mathbb{R}_{+}\right)$and $t<\tau_{S}(x)$ then $t=\tau_{S}(x)-\tau_{S}(\tilde{\pi}(x, t))$.

(b) If $x \in \tilde{F}\left(S, \mathbb{R}_{+}\right)$and $t \geqslant \tau_{S}(x)$ then $t=\tau_{S}(\tilde{\pi}(x, t))+\tau_{S}(x)$.

(c) If $x \in \tilde{\pi}\left(S, \mathbb{R}_{+}\right)$then $t=\tau_{S}(\tilde{\pi}(x, t))-\tau_{S}(x)$.

Proof. (a) Let $x \in \tilde{F}\left(S, \mathbb{R}_{+}\right)$and assume that $t<\tau_{S}(x)$. Thus,

$$
\tilde{\pi}\left(\tilde{\pi}(x, t), \tau_{S}(x)-t\right)=\tilde{\pi}\left(x, \tau_{S}(x)\right) \in S,
$$

that is, $\tilde{\pi}(x, t) \in \tilde{F}\left(S, \mathbb{R}_{+}\right)$. Consequently,

$$
\tilde{\pi}\left(x, t+\tau_{S}(\tilde{\pi}(x, t))\right)=\tilde{\pi}\left(\tilde{\pi}(x, t), \tau_{S}(\tilde{\pi}(x, t))\right) \in S,
$$

which implies $t+\tau_{S}(\tilde{\pi}(x, t))=\tau_{S}(x)$.

(b) Assume that $x \in \tilde{F}\left(S, \mathbb{R}_{+}\right)$and $t \geqslant \tau_{S}(x)$. In this case, one can find $w_{x} \in S$ such that $\tilde{\pi}\left(x, \tau_{S}(x)\right)=w_{x}$. Since $t \geqslant \tau_{S}(x)$, we obtain

$$
\tau_{S}(\tilde{\pi}(x, t))=\tau_{S}\left(\tilde{\pi}\left(\tilde{\pi}\left(x, \tau_{S}(x)\right), t-\tau_{S}(x)\right)\right)=\tau_{S}\left(\tilde{\pi}\left(w_{x}, t-\tau_{S}(x)\right)\right)=t-\tau_{S}(x) .
$$

(c) Suppose that $x \in \tilde{\pi}\left(S, \mathbb{R}_{+}\right)$and let $t \in \mathbb{R}_{+}$. Now, take $z_{x} \in S$ such that $x=\tilde{\pi}\left(z_{x}, \tau_{S}(x)\right)$. Then $\tau_{S}(\tilde{\pi}(x, t))=\tau_{S}\left(\tilde{\pi}\left(z_{x}, \tau_{S}(x)+t\right)\right)=\tau_{S}(x)+t$.

The next lemma deals with the openness of the sets $\tilde{\pi}(S,(0, \infty))$ and $\tilde{F}(S,(0, \infty)) \backslash M$ when $S$ is a section. 
Lemma 4.2.6. Let $(X, \pi, M, I)$ be an ISS such that each element of $M$ satisfies the condition (C) and $I(M) \bigcap M=\emptyset$. Assume that $(X, \pi, M, I)$ admits a section $S$ with section function $\tau_{S}$ continuous on $X \backslash M$. The following conditions hold:

(a) $\tilde{\pi}(S,(0, \infty))$ is open in $X$;

(b) $\tilde{F}(S,(0, \infty)) \backslash M$ is open in $X$.

Proof. (a) Suppose to the contrary that there are $x \in \tilde{\pi}(S,(0, \infty))$ and a sequence $x_{n} \stackrel{n \rightarrow \infty}{\longrightarrow} x$ such that $x_{n} \notin \tilde{\pi}(S,(0, \infty)), n \in \mathbb{N}$. By Lemma 4.2.3, $x \notin M$, thus, we may assume that $x_{n} \notin M$ for all $n \in \mathbb{N}$. Note that $x=\tilde{\pi}\left(z_{x}, \tau_{S}(x)\right)$ with $z_{x} \in S$ and $\tau_{S}(x)>0$. Since $S$ is a section, we have $\left\{x_{n}\right\}_{n \in \mathbb{N}} \subset$ $\tilde{F}\left(S, \mathbb{R}_{+}\right) \backslash M$. Thus, there is $v_{n} \in S$ such that $\tilde{\pi}\left(x_{n}, \tau_{S}\left(x_{n}\right)\right)=v_{n}, n \in \mathbb{N}$. Since $\tau_{S}$ is continuous on $X \backslash M, \tau_{S}\left(x_{n}\right) \stackrel{n \rightarrow \infty}{\longrightarrow} \tau_{S}(x)$. By Lemma 4.2.3, $\tau_{S}(x)$ is a non-jump time of $x$ as $x \in \tilde{\pi}(S,(0, \infty))$. Using Lemma 4.1.5, we get

$$
\tilde{\pi}\left(x_{n}, \tau_{S}\left(x_{n}\right)\right) \stackrel{n \rightarrow \infty}{\longrightarrow} \tilde{\pi}\left(x, \tau_{S}(x)\right)=\tilde{\pi}\left(z_{x}, 2 \tau_{S}(x)\right) .
$$

Note that $\tilde{\pi}\left(z_{x}, 2 \tau_{S}(x)\right) \notin M$ as $I(M) \bigcap M=\emptyset$. Since $\left\{v_{n}\right\}_{n \in \mathbb{N}} \subset S$,

$$
0=\tau_{S}\left(v_{n}\right)=\tau_{S}\left(\tilde{\pi}\left(x_{n}, \tau_{S}\left(x_{n}\right)\right)\right) \stackrel{n \rightarrow \infty}{\longrightarrow} \tau_{S}\left(\tilde{\pi}\left(z_{x}, 2 \tau_{S}(x)\right)\right)=2 \tau_{S}(x),
$$

which is an absurd as $\tau_{S}(x)>0$. Therefore, $\tilde{\pi}(S,(0, \infty))$ is open in $X$.

(b) Suppose to the contrary that there are $x \in \tilde{F}(S,(0, \infty)) \backslash M$ and a sequence $x_{n} \stackrel{n \rightarrow \infty}{\longrightarrow} x$ such that $x_{n} \notin \tilde{F}(S,(0, \infty)) \backslash M, n \in \mathbb{N}$. Since $S$ is a section, we get $\left\{x_{n}\right\}_{n \in \mathbb{N}} \subset \tilde{\pi}(S,[0, \infty)) \backslash M$. Let $\xi_{x} \in S$ be such that $\tilde{\pi}\left(x, \tau_{S}(x)\right)=\xi_{x}$ and let $a_{n} \in S$ be such that $\tilde{\pi}\left(a_{n}, \tau_{S}\left(x_{n}\right)\right)=x_{n}, n \in \mathbb{N}$. By Lemma 4.1.5, there exists a sequence $\left\{\beta_{n}\right\}_{n \in \mathbb{N}}$ in $\mathbb{R}_{+}$such that $\beta_{n} \stackrel{n \rightarrow \infty}{\longrightarrow} 0$ and

$$
\tilde{\pi}\left(a_{n}, \tau_{S}\left(x_{n}\right)+\tau_{S}(x)+\beta_{n}\right)=\tilde{\pi}\left(x_{n}, \tau_{S}(x)+\beta_{n}\right) \stackrel{n \rightarrow \infty}{\longrightarrow} \tilde{\pi}\left(x, \tau_{S}(x)\right)=\xi_{x} \in S .
$$

Consequently, by the continuity of $\tau_{S}$ on $X \backslash M$, we have $\tau_{S}\left(\tilde{\pi}\left(a_{n}, \tau_{S}\left(x_{n}\right)+\tau_{S}(x)+\beta_{n}\right)\right) \stackrel{n \rightarrow \infty}{\longrightarrow} 0$. But, by Lemma 4.2.5,

$$
\tau_{S}\left(\tilde{\pi}\left(a_{n}, \tau_{S}\left(x_{n}\right)+\tau_{S}(x)+\beta_{n}\right)\right)=\tau_{S}\left(x_{n}\right)+\tau_{S}(x)+\beta_{n},
$$

which leads us to $\tau_{S}(x)=0$ which is a contradiction as $x \notin S$. Hence, $\tilde{F}(S,(0, \infty)) \backslash M$ is open in $X$.

Using a section function, we may construct a function $h$ which satisfies conditions $(i i)$ and (iii) from Definition 4.2.2. This result is stated next and its proof is straightforward. 
Lemma 4.2.7. Let $S$ be a section for the ISS $(X, \pi, M, I)$. The function $h: X \rightarrow S \times \mathbb{R}_{+}$given by

$$
h(x)= \begin{cases}\left(\tilde{\pi}\left(x, \tau_{S}(x)\right), \tau_{S}(x)\right) & \text { if } \quad x \in \tilde{F}\left(S, \mathbb{R}_{+}\right), \\ \left(z_{x}, \tau_{S}(x)\right) & \text { if } x \in \tilde{\pi}\left(S, \mathbb{R}_{+}\right),\end{cases}
$$

satisfies conditions (ii) and (iii) from Definition 4.2.2.

\subsection{Parallelizability versus sections}

This section concerns the relationship between parallelizable impulsive semidynamical systems and the existence of sections.

It is well known that a parallelizable dynamical system admits a section with continuous section function, see for instance, Theorem 2.4, Chapter IV, in (BHATIA; SZEGÖ, 2002). In Theorem 3.2.1, we prove this fact in the case of semidynamical systems. Next, we generalize this fact for impulsive semidynamical systems.

Theorem 4.3.1. Let $(X, \pi, M, I)$ be a parallelizable ISS with base $S$. Then $S$ is a section with associated section function $\tau_{S}$ continuous on $X \backslash M$.

Proof. By Definition 4.2.2, $\bar{S} \bigcap M=\emptyset, X=\tilde{F}\left(S, \mathbb{R}_{+}\right) \cup \tilde{\pi}\left(S, \mathbb{R}_{+}\right)$and there exists a function $h: X \rightarrow$ $S \times \mathbb{R}_{+}$satisfying conditions $(i),(i i)$ and $(i i i)$.

We claim that $S$ is a section. Indeed, consider the sets $A=\{w \in X \backslash S: \tilde{F}(w,(0, \infty)) \bigcap S \neq \emptyset\}$ and $B=\{w \in X \backslash S: \tilde{\pi}(w,(0, \infty)) \bigcap S \neq \emptyset\}$.

Let us show that $A$ is nonempty. Indeed, let $a \in S$. Suppose there exists $t>0$ such that $\tilde{\pi}(a, t) \in S$. Using condition (ii) of Definition 4.2.2, we have

$$
(\tilde{\pi}(a, t), 0)=h(\tilde{\pi}(\tilde{\pi}(a, t), 0))=h(\tilde{\pi}(a, t))=(a, t)
$$

which is a contradiction. Hence, $\tilde{\pi}(a, t) \notin S$ for all $t>0$. This shows that $\tilde{\pi}(a, t) \in A$ for all $a \in S$ and $t>0$.

Let $x \in A$. If there are $\lambda_{1}, \lambda_{2} \in \mathbb{R}_{+}$and $x_{1}, x_{2} \in S$ such that $\tilde{\pi}\left(x_{1}, \lambda_{1}\right)=x$ and $\tilde{\pi}\left(x_{2}, \lambda_{2}\right)=x$, then it follows by condition (ii) of Definition 4.2.2 that $\left(x_{1}, \lambda_{1}\right)=h(x)=\left(x_{2}, \lambda_{2}\right)$, that is, $x_{1}=x_{2}$ and $\lambda_{1}=\lambda_{2}$. Hence, for each $x \in A$, there exist unique $z_{x} \in S$ and $t_{x}>0$ such that

$$
\tilde{F}(x,(0, \infty)) \bigcap S=\tilde{F}\left(x, t_{x}\right\}=\left\{z_{x}\right\} .
$$


On the other hand, let $x \in A$ and suppose that $\tilde{\pi}(x, t)=w \in S$ for some $t \geqslant 0$. We have just proved that there exist $z_{x} \in S$ and $t_{x}>0$ such that $\tilde{\pi}\left(z_{x}, t_{x}\right)=x$. Then $\tilde{\pi}\left(z_{x}, t_{x}+t\right)=w$, that is, $z_{x} \in \tilde{F}\left(w, t_{x}+t\right)$. By condition (iii) of Definition 4.2.2,

$$
\left(z_{x}, 0\right)=h\left(z_{x}\right)=\left(w, t_{x}+t\right),
$$

which leads us to a contradiction. Hence, $\tilde{\pi}\left(x, \mathbb{R}_{+}\right) \bigcap S=\emptyset$.

Analogously, we can prove that $B \neq \emptyset$ and, for each $x \in B$, there exists a unique $t_{x}>0$ such that $\tilde{\pi}\left(x, t_{x}\right) \in S$ and $\tilde{F}\left(x, \mathbb{R}_{+}\right) \bigcap S=\emptyset$.

Hence, $S$ is a section.

Now, let $\tau_{S}: X \rightarrow \mathbb{R}_{+}$be the section function associated with $S$ (as defined in (4.2.1)). Consider the function $g: X \rightarrow S \times \mathbb{R}_{+}$given by

$$
g(x)= \begin{cases}\left(\tilde{\pi}\left(x, \tau_{S}(x)\right), \tau_{S}(x)\right) & \text { if } \quad x \in \tilde{F}\left(S, \mathbb{R}_{+}\right) \\ \left(z_{x}, \tau_{S}(x)\right) & \text { if } x \in \tilde{\pi}\left(S, \mathbb{R}_{+}\right)\end{cases}
$$

By Lemma 4.2.7, the function $g$ satisfies conditions (ii) and (iii) from Definition 4.2.2. We claim that $h(x)=g(x)$ for all $x \in X$. Indeed, if $x \in \tilde{F}\left(S, \mathbb{R}_{+}\right)$then $\tilde{\pi}\left(x, \tau_{S}(x)\right)=y \in S$. Thus,

$$
g(x)=\left(\tilde{\pi}\left(x, \tau_{S}(x)\right), \tau_{S}(x)\right)=\left(y, \tau_{S}(x)\right)=h(x) .
$$

On the other hand, if $x \in \tilde{\pi}\left(S, \mathbb{R}_{+}\right)$then $x=\tilde{\pi}\left(z_{x}, \tau_{S}(x)\right)$ and $g(x)=\left(z_{x}, \tau_{S}(x)\right)=h(x)$. Since $X=\tilde{F}\left(S, \mathbb{R}_{+}\right) \cup \tilde{\pi}\left(S, \mathbb{R}_{+}\right)$, we conclude that $h(x)=g(x)$ for all $x \in X$.

Hence, $g$ is continuous on $X \backslash M$ since $h$ possesses this property. Consequently, we conclude that $\tau_{S}$ is continuous on $X \backslash M$.

The converse of Theorem 4.3.1 does not hold in general. In Theorem 4.3.2 we give sufficient conditions to obtain this reciprocal. Before that, we present an auxiliary result.

Lemma 4.3.1. Assume that $(X, \pi, M, I)$ is an ISS such that $I(M) \bigcap M=\varnothing$ and each point of $M$ satisfies condition (C). Let $S$ be a compact section with $\tau_{S}$ continuous on $X \backslash M$. Let $x, y \in X \backslash M$ and $\left\{x_{n}\right\}_{n \in \mathbb{N}},\left\{y_{n}\right\}_{n \in \mathbb{N}}$ be sequences in $X$ such that $x_{n} \stackrel{n \rightarrow \infty}{\longrightarrow} x$ and $y_{n} \stackrel{n \rightarrow \infty}{\longrightarrow} y$.

(a) If $x_{n} \in \tilde{F}\left(S, \mathbb{R}_{+}\right)$for every $n \in \mathbb{N}$, then $\tilde{\pi}\left(x_{n}, \tau_{S}\left(x_{n}\right)\right) \stackrel{n \rightarrow \infty}{\longrightarrow} \tilde{\pi}\left(x, \tau_{S}(x)\right)$.

(b) If $\tilde{\pi}\left(y_{n}, \tau_{S}\left(x_{n}\right)\right)=x_{n}$ for every $n \in \mathbb{N}$, then $x=\tilde{\pi}\left(y, \tau_{S}(x)\right)$. 
Proof. (a) Since $x \notin M$, it follows by Lemma 4.1.5 that there exists a sequence $\left\{\varepsilon_{n}\right\}_{n \in \mathbb{N}} \subset \mathbb{R}_{+}$ such that $\varepsilon_{n} \stackrel{n \rightarrow \infty}{\longrightarrow} 0$ and

$$
\tilde{\pi}\left(x_{n}, \tau_{S}\left(x_{n}\right)+\varepsilon_{n}\right) \stackrel{n \rightarrow \infty}{\longrightarrow} \tilde{\pi}\left(x, \tau_{S}(x)\right) .
$$

Let $\left\{\tilde{\pi}\left(x_{n_{k}}, \tau_{S}\left(x_{n_{k}}\right)\right)\right\}_{k \in \mathbb{N}}$ be an arbitrary subsequence of $\left\{\tilde{\pi}\left(x_{n}, \tau_{S}\left(x_{n}\right)\right)\right\}_{n \in \mathbb{N}}$. By compactness of $S$ and by the fact that $\tilde{\pi}\left(x_{n_{k}}, \tau_{S}\left(x_{n_{k}}\right)\right) \in S, k \in \mathbb{N}$, we may assume without loss of generality that $\tilde{\pi}\left(x_{n_{k}}, \tau_{S}\left(x_{n_{k}}\right)\right) \stackrel{n \rightarrow \infty}{\longrightarrow} z \in S$. But, as $S \bigcap M=\emptyset$ it follows by Lemma 4.1 .4 that

$$
\tilde{\pi}\left(x_{n_{k}}, \tau_{S}\left(x_{n_{k}}\right)+\varepsilon_{n_{k}}\right) \stackrel{k \rightarrow \infty}{\longrightarrow} z .
$$

Consequently, by (4.3.2), $z=\tilde{\pi}\left(x, \tau_{S}(x)\right)$. This shows that the entire sequence $\left\{\tilde{\pi}\left(x_{n}, \tau_{S}\left(x_{n}\right)\right)\right\}_{n \in \mathbb{N}}$ converges to $\tilde{\pi}\left(x, \tau_{S}(x)\right)$.

(b) Since $y \notin M$, it follows by Lemma 4.1.5 that there is a sequence $\left\{\varepsilon_{n}\right\}_{n \in \mathbb{N}} \subset \mathbb{R}_{+}$such that $\varepsilon_{n} \stackrel{n \rightarrow \infty}{\longrightarrow} 0$ and

$$
\tilde{\pi}\left(y_{n}, \tau_{S}\left(x_{n}\right)+\varepsilon_{n}\right) \stackrel{k \rightarrow \infty}{\longrightarrow} \tilde{\pi}\left(y, \tau_{S}(x)\right) .
$$

Note that $\tilde{\pi}\left(y_{n}, \tau_{S}\left(x_{n}\right)\right)=x_{n} \stackrel{n \rightarrow \infty}{\longrightarrow} x$ and $x \notin M$, which leads to $\tilde{\pi}\left(y_{n}, \tau_{S}\left(x_{n}\right)+\varepsilon_{n}\right) \stackrel{n \rightarrow \infty}{\longrightarrow} x$ (see Lemma 4.1.4). Hence, $x=\tilde{\pi}\left(y, \tau_{S}(x)\right)$.

Theorem 4.3.2. Let $(X, \pi, M, I)$ be an ISS such that $I(M) \bigcap M=\varnothing$ and each point of $M$ satisfies condition (C). Assume that $(X, \pi, M, I)$ admits a compact section $S$ with section function $\tau_{S}$ continuous on $X \backslash M$. Then $(X, \pi, M, I)$ is parallelizable.

Proof. Since $S$ is a section, we have $X=\tilde{F}\left(S, \mathbb{R}_{+}\right) \cup \tilde{\pi}\left(S, \mathbb{R}_{+}\right)$. According to the Lemma 4.2.7, it is enough to show that the function $h$ given in (4.2.2) is continuous in $X \backslash M=\left[\tilde{F}\left(S, \mathbb{R}_{+}\right) \cup \tilde{\pi}\left(S, \mathbb{R}_{+}\right)\right] \backslash M$. Let $x \in X \backslash M$ and $\left\{x_{n}\right\}_{n \in \mathbb{N}} \subset X$ be a sequence such that $x_{n} \stackrel{n \rightarrow \infty}{\longrightarrow} x$.

Case 1: $x \in \tilde{F}(S,(0, \infty)) \backslash M$.

Let $\xi_{x} \in S$ be such that $\pi\left(x, \tau_{S}(x)\right)=\xi_{x}$. By Lemma 4.2.6, we may assume without loss of generality that $\left\{x_{n}\right\}_{n \in \mathbb{N}} \subset \tilde{F}(S,(0, \infty)) \backslash M$. In this way, using condition $(a)$ from Lemma 4.3.1, we obtain

$$
h\left(x_{n}\right)=\left(\tilde{\pi}\left(x_{n}, \tau_{S}\left(x_{n}\right)\right), \tau_{S}\left(x_{n}\right)\right) \stackrel{n \rightarrow \infty}{\longrightarrow}\left(\tilde{\pi}\left(x, \tau_{S}(x)\right), \tau_{S}(x)\right)=h(x) .
$$

Case 2: $x \in \tilde{\pi}(S,(0, \infty)) \backslash M$.

Let $z_{x} \in S$ be such that $x=\tilde{\pi}\left(z_{x}, \tau_{S}(x)\right)$. Note that $\tau_{S}(x)>0$. By Lemma 4.2.6, we may assume that $\left\{x_{n}\right\}_{n \in \mathbb{N}} \subset \tilde{\pi}(S,(0, \infty)) \backslash M$. Consequently, one can find $z_{x_{n}} \in S$ such that $\tilde{\pi}\left(z_{x_{n}}, \tau_{S}\left(x_{n}\right)\right)=$ 
$x_{n}$ for each $n \in \mathbb{N}$. Now we claim that $z_{x_{n}} \stackrel{n \rightarrow \infty}{\longrightarrow} z_{x}$. In fact, let $\left\{z_{x_{n_{k}}}\right\}_{k \in \mathbb{N}}$ be an arbitrary subsequence of $\left\{z_{x_{n}}\right\}_{n \in \mathbb{N}}$. Since $S$ is compact, we may assume that $z_{x_{n_{k}}} \stackrel{k \rightarrow \infty}{\longrightarrow} w$ for some $w \in S$. By Lemma 4.3.1, item $(b)$,

$$
x_{n_{k}}=\tilde{\pi}\left(z_{x_{n_{k}}}, \tau_{S}\left(x_{n_{k}}\right)\right) \stackrel{k \rightarrow \infty}{\longrightarrow} \tilde{\pi}\left(w, \tau_{S}(x)\right)=x .
$$

Since $S$ is a section, we have $w=z_{x}$. Hence, $\left\{z_{x_{n}}\right\}_{n \in \mathbb{N}}$ converges to $z_{x}$. Thus,

$$
h\left(x_{n}\right)=\left(z_{x_{n}}, \tau_{S}\left(x_{n}\right)\right) \stackrel{n \rightarrow \infty}{\longrightarrow}\left(z_{x}, \tau_{S}(x)\right)=h(x) .
$$

Case 3: $x \in S$.

Here, $\tau_{S}(x)=0$. If $\left\{x_{n}\right\}_{n \in \mathbb{N}}$ admits a subsequence $\left\{x_{n_{k}}\right\}_{k \in \mathbb{N}} \subset \tilde{F}\left(S, \mathbb{R}_{+}\right)$then using the fact that $x \notin M, \tau_{S}\left(x_{n_{k}}\right) \stackrel{k \rightarrow \infty}{\longrightarrow} 0$ and Lemma 4.1.4, we get

$$
h\left(x_{n_{k}}\right)=\left(\tilde{\pi}\left(x_{n_{k}}, \tau_{S}\left(x_{n_{k}}\right)\right), \tau_{S}\left(x_{n_{k}}\right)\right) \stackrel{k \rightarrow \infty}{\longrightarrow}(x, 0)=h(x) .
$$

If $\left\{x_{n}\right\}_{n \in \mathbb{N}}$ admits a subsequence $\left\{x_{n_{m}}\right\}_{m \in \mathbb{N}} \subset \tilde{\pi}\left(S, \mathbb{R}_{+}\right)$then there exists $\left\{z_{x_{n_{m}}}\right\}_{m \in \mathbb{N}} \subset S$ such that $\tilde{\pi}\left(z_{x_{n}}, \tau_{S}\left(x_{n_{m}}\right)\right)=x_{n_{m}}$ for all $m \in \mathbb{N}$. Since $x_{n_{m}} \stackrel{m \rightarrow \infty}{\longrightarrow} x, \tau_{S}\left(x_{n_{m}}\right) \stackrel{m \rightarrow \infty}{\longrightarrow} 0$ and $S$ is compact, by using the equality $\tilde{\pi}\left(z_{x_{n_{m}}}, \tau_{S}\left(x_{n_{m}}\right)\right)=x_{n_{m}}$, it follows that every subsequence of $\left\{z_{x_{n_{m}}}\right\}_{m \in \mathbb{N}}$ admits an extra subsequence which converges to $x$. Thus, $z_{x_{n_{m}}} \stackrel{m \rightarrow \infty}{\longrightarrow} x$ and

$$
h\left(x_{n_{m}}\right)=\left(z_{x_{n_{m}}}, \tau_{S}\left(x_{n_{m}}\right)\right) \stackrel{m \rightarrow \infty}{\longrightarrow}(x, 0)=h(x) .
$$

This concludes the proof and $(X, \pi, M, I)$ is parallelizable.

We end this section with the following result concerning stationary points and periodic points.

Theorem 4.3.3. Let $(X, \pi, M, I)$ be a parallelizable ISS with section $S$. Then there exist neither stationary points nor periodic points in $\left(X, \pi, \mathbb{R}_{+}\right)$.

Proof. By the definition of section, a parallelizable ISS cannot admit stationary points.

On the other hand, assume that $(X, \pi, M, I)$ admits a periodic point $x$ in $(X, \pi, M, I)$ with period $T>0$. If $x \in \tilde{F}\left(S, \mathbb{R}_{+}\right)$then $\tilde{\pi}\left(x, \tau_{S}(x)\right) \in S$. Consequently, $\tilde{\pi}\left(x, \tau_{S}(x)+T\right)=\tilde{\pi}\left(x, \tau_{S}(x)\right) \in S$ which implies $\tau_{S}(x)=\tau_{S}(x)+T$ as $S$ is a section. But this is a contradiction because $T \neq 0$.

If $x \in \tilde{\pi}\left(S, \mathbb{R}_{+}\right)$, then there is $z_{x} \in S$ such that $\tilde{\pi}\left(z_{x}, \tau_{S}(x)\right)=x=\tilde{\pi}\left(z_{x}, \tau_{S}(x)+T\right)$, thus $T=0$, which is again a contradiction. In this way, there is no periodic point in $(X, \pi, M, I)$. 


\subsection{Parallelizability versus dispersivity}

This section concerns the relationship between parallelizable impulsive semidynamical systems and dispersive impulsive semidynamical systems.

Analogously to the Definition 3.3.1, we define the concept of dispersiveness for impulsive semidynamical system by using the notion of impulsive positive prolongational limit sets, see Definition 2.2.10.

Definition 4.4.1. An ISS $(X, \pi, M, I)$ is called dispersive if $\tilde{J}^{+}(x)=\emptyset$ for all $x \in X$.

Example 4.4.1. In Example 4.1.2, if $I(M) \subset B((0,0), 1)$ then $\left(\mathbb{R}^{2}, \pi, M, I\right)$ is dispersive.

In Theorem 4.4.1, we prove that every parallelizable ISS is also dispersive. Before that, we show an auxiliary result.

Lemma 4.4.1. Let $(X, \pi, M, I)$ be a parallelizable ISS with base $S$ such that each element of $M$ satisfies the condition (C) and $I(M) \cap M=\emptyset$. Let $x \in M$ and $\left\{x_{n}\right\}_{n \in \mathbb{N}} \subset X$ be a sequence such that $x_{n} \stackrel{n \rightarrow \infty}{\longrightarrow} x$. Then $\limsup _{n \rightarrow \infty} \tau_{S}\left(x_{n}\right)<\infty$.

Proof. Suppose to the contrary that there exists a subsequence of $\left\{x_{n}\right\}_{n \in \mathbb{N}}$, which we denote by the same, such that $\tau_{S}\left(x_{n}\right) \stackrel{n \rightarrow \infty}{\longrightarrow} \infty$. By Corollary 4.1.1, there exists a subsequence $\left\{x_{n_{k}}\right\}_{k \in \mathbb{N}}$ of $\left\{x_{n}\right\}_{n \in \mathbb{N}}$ such that either $\phi\left(x_{n_{k}}\right) \stackrel{k \rightarrow \infty}{\longrightarrow} 0$ or $\phi\left(x_{n_{k}}\right) \stackrel{k \rightarrow \infty}{\longrightarrow} \phi(x)$.

Case 1: $\phi\left(x_{n_{k}}\right) \stackrel{k \rightarrow \infty}{\longrightarrow} 0$.

In this case, $\tilde{\pi}\left(x_{n_{k}}, \phi\left(x_{n_{k}}\right)\right) \stackrel{k \rightarrow \infty}{\longrightarrow} I(x)$. Since $I(x) \notin M$ (because $I(M) \bigcap M=\emptyset$ ) and $\tau_{S}$ is continuous on $X \backslash M$ (see Theorem 4.3.1), we get

$$
\tau_{S}\left(\tilde{\pi}\left(x_{n_{k}}, \phi\left(x_{n_{k}}\right)\right)\right) \stackrel{k \rightarrow \infty}{\longrightarrow} \tau_{S}(I(x)) .
$$

If $\left\{x_{n_{k}}\right\}_{k \in \mathbb{N}} \subset \tilde{F}\left(S, \mathbb{R}_{+}\right)$(up to a subsequence) then by Lemma 4.2.5, condition $(a)$, we have $\tau_{S}\left(\tilde{\pi}\left(x_{n_{k}}, \phi\left(x_{n_{k}}\right)\right)\right)=\tau_{S}\left(x_{n_{k}}\right)-\phi\left(x_{n_{k}}\right) \stackrel{k \rightarrow \infty}{\longrightarrow} \infty$ which contradicts (4.4.1).

Now, if $\left\{x_{n_{k}}\right\}_{k \in \mathbb{N}} \subset \tilde{\pi}\left(S, \mathbb{R}_{+}\right)$(up to a subsequence) then by Lemma 4.2.5, condition (c), we have $\tau_{S}\left(\tilde{\pi}\left(x_{n_{k}}, \phi\left(x_{n_{k}}\right)\right)\right)=\tau_{S}\left(x_{n_{k}}\right)+\phi\left(x_{n_{k}}\right) \stackrel{k \rightarrow \infty}{\longrightarrow} \infty$ which contradicts (4.4.1).

Case 2: $\phi\left(x_{n_{k}}\right) \stackrel{k \rightarrow \infty}{\longrightarrow} \phi(x)$. 
In this case, $\tilde{\pi}\left(x_{n_{k}}, s\right) \stackrel{k \rightarrow \infty}{\longrightarrow} \tilde{\pi}(x, s)$ for $s \in(0, \phi(x))$. Thus

$$
\tau_{S}\left(\tilde{\pi}\left(x_{n_{k}}, s\right)\right) \stackrel{k \rightarrow \infty}{\longrightarrow} \tau_{S}(\tilde{\pi}(x, s))
$$

as $\tilde{\pi}(x, s) \notin M$ (because $I(M) \bigcap M=\emptyset$ ) and $\tau_{S}$ is continuous on $X \backslash M$ by Theorem 4.3.1.

If $\left\{x_{n_{k}}\right\}_{k \in \mathbb{N}} \subset \tilde{F}\left(S, \mathbb{R}_{+}\right)$(up to a subsequence), then by Lemma 4.2.5, condition (a), we get $\tau_{S}\left(\tilde{\pi}\left(x_{n_{k}}, s\right)\right)=\tau_{S}\left(x_{n_{k}}\right)-s \stackrel{k \rightarrow \infty}{\longrightarrow} \infty$ and it contradicts (4.4.2).

If $\left\{x_{n_{k}}\right\}_{k \in \mathbb{N}} \subset \tilde{\pi}\left(S, \mathbb{R}_{+}\right)$(up to a subsequence), then by Lemma 4.2.5, condition (c), we obtain $\tau_{S}\left(\tilde{\pi}\left(x_{n_{k}}, s\right)\right)=\tau_{S}\left(x_{n_{k}}\right)+s \stackrel{k \rightarrow \infty}{\longrightarrow} \infty$ which contradicts (4.4.2).

Therefore, we conclude that $\lim \sup \tau_{S}\left(x_{n}\right)<\infty$.

Theorem 4.4.1. Let $(X, \pi, M, I)$ be a parallelizable ISS with base $S$ such that each element of $M$ satisfies the condition $(\mathrm{C})$ and $I(M) \cap M=\emptyset$. Then $(X, \pi, M, I)$ is dispersive.

Proof. Suppose to the contradiction that there are $x \in X$ and $y \in \tilde{J}^{+}(x)$. Then there are sequences $\left\{x_{n}\right\}_{n \in \mathbb{N}} \subset X$ and $\left\{t_{n}\right\}_{n \in \mathbb{N}} \subset \mathbb{R}_{+}$such that $x_{n} \stackrel{n \rightarrow \infty}{\longrightarrow} x, t_{n} \stackrel{n \rightarrow \infty}{\longrightarrow \infty}$ and $\tilde{\pi}\left(x_{n}, t_{n}\right) \stackrel{n \rightarrow \infty}{\longrightarrow} y$. Since $X=$ $\tilde{F}\left(S, \mathbb{R}_{+}\right) \cup \tilde{\pi}\left(S, \mathbb{R}_{+}\right)$, we can decompose $\left\{x_{n}\right\}_{n \in \mathbb{N}}$ into $\left\{x_{n}\right\}_{n \in \mathbb{N}}=\left\{x_{k}\right\}_{k \in \mathbb{N}_{1}} \cup\left\{x_{\ell}\right\}_{\ell \in \mathbb{N}_{2}}$, where $\mathbb{N}=$ $\mathbb{N}_{1} \cup \mathbb{N}_{2}$,

$$
\left\{x_{k}\right\}_{k \in \mathbb{N}_{1}} \subset \tilde{F}\left(S, \mathbb{R}_{+}\right) \quad \text { and } \quad\left\{x_{\ell}\right\}_{\ell \in \mathbb{N}_{2}} \subset \tilde{\pi}\left(S, \mathbb{R}_{+}\right) .
$$

Recall by Theorem 4.3.1 that $\tau_{S}$ is continuous on $X \backslash M$.

Case 1: $x \notin M$ and $y \notin M$.

Here, $\tau_{S}\left(x_{n}\right) \stackrel{n \rightarrow \infty}{\longrightarrow} \tau_{S}(x)$ and $\tau_{S}\left(\tilde{\pi}\left(x_{n}, t_{n}\right)\right) \stackrel{n \rightarrow \infty}{\longrightarrow} \tau_{S}(y)$. If $\mathbb{N}_{1}$ is a countably infinite set, then by using Lemma 4.2 .5 , case $(b)$, we get $t_{k}=\tau_{S}\left(\tilde{\pi}\left(x_{k}, t_{k}\right)\right)+\tau_{S}\left(x_{k}\right)$ for $k \in \mathbb{N}_{1}$ sufficiently large. This shows that $\left\{t_{k}\right\}_{k \in \mathbb{N}_{1}}$ is convergent which is a contradiction.

If $\mathbb{N}_{2}$ is a countably infinite set then, by Lemma 4.2.5, case $(c)$, we conclude that $t_{\ell}=$ $\tau_{S}\left(\tilde{\pi}\left(x_{\ell}, t_{\ell}\right)\right)-\tau_{S}\left(x_{\ell}\right)$ for every $\ell \in \mathbb{N}_{2}$. Again, we conclude that $\left\{t_{\ell}\right\}_{\ell \in \mathbb{N}_{2}}$ is convergent and it is a contradiction.

Case 2: $x \notin M$ and $y \in M$.

By Lemma 4.4.1 we have $\limsup _{n \rightarrow \infty} \tau_{S}\left(\tilde{\pi}\left(x_{n}, t_{n}\right)\right)<\infty$ and by the continuity of $\tau_{S}$ on $X \backslash M$, we get $\tau_{S}\left(x_{n}\right) \stackrel{n \rightarrow \infty}{\longrightarrow} \tau_{S}(x)$. Up to a subsequence, we may assume without loss of generality that $\tau_{S}\left(x_{n}\right)<$ $t_{n}$ for all $n \in \mathbb{N}$. Consequently, by Lemma 4.2 .5 , we obtain $\tau_{S}\left(\tilde{\pi}\left(x_{k}, t_{k}\right)\right)=t_{k}-\tau_{S}\left(x_{k}\right) \stackrel{k \rightarrow \infty}{\longrightarrow} \infty$, if $\mathbb{N}_{1}$ 
is a countably infinite set, and $\tau_{S}\left(\tilde{\pi}\left(x_{\ell}, t_{\ell}\right)\right)=t_{\ell}+\tau_{S}\left(x_{\ell}\right) \stackrel{\ell \rightarrow \infty}{\longrightarrow} \infty, \mathbb{N}_{2}$ is a countably infinite set. Thus, we have a contradiction.

Case 3: $x \in M$ and $y \notin M$.

In this case, we have $\limsup _{n \rightarrow \infty} \tau_{S}\left(x_{n}\right)<\infty$ and $\tau_{S}\left(\tilde{\pi}\left(x_{n}, t_{n}\right)\right) \stackrel{n \rightarrow \infty}{\longrightarrow} \tau_{S}(y)$. But, according to Lemma 4.2.5, we obtain $\tau_{S}\left(\tilde{\pi}\left(x_{k}, t_{k}\right)\right)=t_{k}-\tau_{S}\left(x_{k}\right) \stackrel{k \rightarrow \infty}{\longrightarrow} \infty$, if $\mathbb{N}_{1}$ is a countably infinite set, and $\tau_{S}\left(\tilde{\pi}\left(x_{\ell}, t_{\ell}\right)\right)=t_{\ell}+\tau_{S}\left(x_{\ell}\right) \stackrel{\ell \rightarrow \infty}{\longrightarrow} \infty, \mathbb{N}_{2}$ is a countably infinite set. Thus, we get again a contradiction.

Case 4: $x \in M$ and $y \in M$.

In this last case, we obtain $\limsup \tau_{S}\left(x_{n}\right)<\infty$ and $\lim \sup \tau_{S}\left(\tilde{\pi}\left(x_{n}, t_{n}\right)\right)<\infty$ (see Lemma 4.4.1). On the other hand, using Lemma 4.2.5, we get the contradictions $\tau_{S}\left(\tilde{\pi}\left(x_{k}, t_{k}\right)\right)=t_{k}-$ $\tau_{S}\left(x_{k}\right) \stackrel{k \rightarrow \infty}{\longrightarrow} \infty$, if $\mathbb{N}_{1}$ is a countably infinite set, and $\tau_{S}\left(\tilde{\pi}\left(x_{\ell}, t_{\ell}\right)\right)=t_{\ell}+\tau_{S}\left(x_{\ell}\right) \stackrel{\ell \rightarrow \infty}{\longrightarrow} \infty, \mathbb{N}_{2}$ is a countably infinite set.

In conclusion, $\tilde{J}^{+}(x)=\emptyset$ for all $x \in X$, that is, $(X, \pi, M, I)$ is dispersive.

Sufficient conditions for a dispersive ISS to be parallelizable are given in next theorem.

Theorem 4.4.2. Let $(X, \pi, M, I)$ be dispersive such that each element of $M$ satisfies the condition (C) and $I(M) \cap M=\emptyset$. If $(X, \pi, M, I)$ admits a compact section $S$, then $(X, \pi, M, I)$ is parallelizable.

Proof. By Lemma 4.2.7, we need to show that the function $h$ given in (4.2.2) is continuous on $X \backslash M=\left[\tilde{F}\left(S, \mathbb{R}_{+}\right) \cup \tilde{\pi}\left(S, \mathbb{R}_{+}\right)\right] \backslash M$. Suppose to the contrary that there is $x \in X \backslash M$ such that $\tau_{S}$ is discontinuous at $x$. Then there are $\varepsilon_{0}>0$ and a sequence $\left\{x_{n}\right\}_{n \in \mathbb{N}} \subset X$ such that $x_{n} \stackrel{n \rightarrow \infty}{\longrightarrow} x$ and

$$
\left|\tau_{S}\left(x_{n}\right)-\tau_{S}(x)\right| \geqslant \varepsilon_{0}
$$

for all $n \in \mathbb{N}$.

Case 1: $\left\{x_{n}\right\}_{n \in \mathbb{N}} \subset \tilde{F}\left(S, \mathbb{R}_{+}\right)$, up to a subsequence.

In this case, $\tilde{\pi}\left(x_{n}, \tau_{S}\left(x_{n}\right)\right) \in S$ for all $n \in \mathbb{N}$. We may assume without loss of generality that $\tilde{\pi}\left(x_{n}, \tau_{S}\left(x_{n}\right)\right) \stackrel{n \rightarrow \infty}{\longrightarrow} y \in S$ as $S$ is compact.

If $\left\{\tau_{S}\left(x_{n}\right)\right\}_{n \in \mathbb{N}}$ admits a convergent subsequence, which we denote by the same, i.e., $\tau_{S}\left(x_{n}\right) \stackrel{n \rightarrow \infty}{\longrightarrow} r$ then, by Lemma 4.1 .5 , there exists a sequence of nonnegative numbers $\varepsilon_{n} \stackrel{n \rightarrow \infty}{\longrightarrow} 0$ such 
that

$$
\tilde{\pi}\left(x_{n}, \tau_{S}\left(x_{n}\right)+\varepsilon_{n}\right) \stackrel{n \rightarrow \infty}{\longrightarrow} \tilde{\pi}(x, r) .
$$

But since $y \in S$ and $S \bigcap M=\emptyset$, we obtain by Lemma 4.1.4 the following convergence

$$
\tilde{\pi}\left(x_{n}, \tau_{S}\left(x_{n}\right)+\varepsilon_{n}\right) \stackrel{n \rightarrow \infty}{\longrightarrow} y .
$$

Hence, $\tilde{\pi}(x, r)=y \in S$. Consequently, $r=\tau_{S}(x)$. Thus, $\tau_{S}\left(x_{n}\right) \stackrel{n \rightarrow \infty}{\longrightarrow} \tau_{S}(x)$ which contradicts (4.4.3).

If $\left\{\tau_{S}\left(x_{n}\right)\right\}_{n \in \mathbb{N}}$ admits an unbounded subsequence $\tau_{S}\left(x_{n}\right) \stackrel{n \rightarrow \infty}{\longrightarrow} \infty$ (up to a subsequence), then $y \in \tilde{J}^{+}(x)$ which contradicts the fact that $(X, \pi, M, I)$ is dispersive.

Case 2: $\left\{x_{n}\right\}_{n \in \mathbb{N}} \subset \tilde{\pi}\left(S, \mathbb{R}_{+}\right)$, up to a subsequence.

In this case, for each $n \in \mathbb{N}$, there is $z_{n} \in S$ such that $\tilde{\pi}\left(z_{n}, \tau_{S}\left(x_{n}\right)\right)=x_{n}$. Since $S$ is compact, we may assume that $z_{n} \stackrel{n \rightarrow \infty}{\longrightarrow} z \in S$. We may proceed as in the proof of Case 1 , which leads us to a contradiction.

Hence, $\tau_{S}$ is continuous on $X \backslash M$ and $(X, \pi, M, I)$ is parallelizable.

Recall from the Definition 3.2.2 that a section $S$ satisfies the condition (T), if for every $z \in S$ and every sequence $\left\{z_{n}\right\}_{n \in \mathbb{N}} \subset X$ such that $z_{n} \stackrel{n \rightarrow \infty}{\longrightarrow} z$ then $\tau_{S}\left(z_{n}\right) \stackrel{n \rightarrow \infty}{\longrightarrow} 0$. The next result deals with the openness of $\tilde{F}(S,(0, \infty)) \backslash M$ using condition (T).

Lemma 4.4.2. Let $(X, \pi, M, I)$ be an ISS such that each element of $M$ satisfies the condition (C) and $I(M) \cap M=\emptyset$. Assume that $(X, \pi, M, I)$ admits a section $S$ satisfying condition (T). Then $\tilde{F}(S,(0, \infty)) \backslash M$ is open in $X$.

Proof. Suppose to the contrary that there are $x \in \tilde{F}(S,(0, \infty)) \backslash M$ and a sequence $x_{n} \stackrel{n \rightarrow \infty}{\longrightarrow} x$ such that $x_{n} \notin \tilde{F}(S,(0, \infty)) \backslash M, n \in \mathbb{N}$. Since $S$ is a section and $M$ is closed, we may assume that $\left\{x_{n}\right\}_{n \in \mathbb{N}} \subset \tilde{\pi}(S,[0, \infty)) \backslash M$. Let $w \in S$ be such that $\tilde{\pi}\left(x, \tau_{S}(x)\right)=w$ and let $a_{n} \in S$ be such that $\tilde{\pi}\left(a_{n}, \tau_{S}\left(x_{n}\right)\right)=x_{n}, n \in \mathbb{N}$. By Lemma 4.1.5, there exists a sequence $\left\{\beta_{n}\right\}_{n \in \mathbb{N}}$ in $\mathbb{R}_{+}$such that $\beta_{n} \stackrel{n \rightarrow \infty}{\longrightarrow} 0$ and

$$
\tilde{\pi}\left(a_{n}, \tau_{S}\left(x_{n}\right)+\tau_{S}(x)+\beta_{n}\right)=\tilde{\pi}\left(x_{n}, \tau_{S}(x)+\beta_{n}\right) \stackrel{n \rightarrow \infty}{\longrightarrow} \tilde{\pi}\left(x, \tau_{S}(x)\right)=w \in S .
$$

Consequently, by condition $(\mathrm{T})$ we have $\tau_{S}\left(\tilde{\pi}\left(a_{n}, \tau_{S}\left(x_{n}\right)+\tau_{S}(x)+\beta_{n}\right)\right) \stackrel{n \rightarrow \infty}{\longrightarrow} 0$. But, by Lemma 4.2.5,

$$
\tau_{S}\left(\tilde{\pi}\left(a_{n}, \tau_{S}\left(x_{n}\right)+\tau_{S}(x)+\beta_{n}\right)\right)=\tau_{S}\left(x_{n}\right)+\tau_{S}(x)+\beta_{n},
$$

which leads us to a contradiction as $\tau_{S}(x)>0(x \notin S)$. Hence, $\tilde{F}(S,(0, \infty)) \backslash M$ is open in $X$. 
Consider the set-valued map $\tilde{F}: X \times \mathbb{R}_{+} \rightarrow X$ given by

$$
\tilde{F}(x, t)=\{y \in X: \tilde{\pi}(y, t)=x\} .
$$

Recall that the Hausdorff semidistance between two nonempty bounded subsets $A$ and $B$ of $X$ is given by $d_{H}(A, B)=\sup \{d(a, B): a \in A\}$.

Definition 4.4.2. The map $\tilde{F}: X \times \mathbb{R}_{+} \rightarrow X$ is said to be upper semicontinuous at $(x, t) \in X \times$ $\mathbb{R}_{+}$, if for all sequences $\left\{x_{n}\right\}_{n \in \mathbb{N}} \subset X$ and $\left\{t_{n}\right\}_{n \in \mathbb{N}} \subset \mathbb{R}_{+}$such that $x_{n} \stackrel{n \rightarrow \infty}{\longrightarrow} x$ and $t_{n} \stackrel{n \rightarrow \infty}{\longrightarrow} t$ then $d_{H}\left(\tilde{F}\left(x_{n}, t_{n}\right), \tilde{F}(x, t)\right) \stackrel{n \rightarrow \infty}{\longrightarrow} 0$. The map $\tilde{F}$ is upper semicontinuous in $A \subset X \times \mathbb{R}_{+}$, if it is upper semicontinuous at each point $(x, t) \in A$.

Theorem 4.4.3. Let $(X, \pi, M, I)$ be an ISS such that each element of $M$ satisfies the condition (C), $I(M) \cap M=\emptyset$ and $I(M) \cap S=\emptyset$. Assume that $(X, \pi, M, I)$ is dispersive with section $S$ satisfying condition (T). If $\tilde{F}(w, s) \neq \emptyset$ for all $w \in X \backslash M$ and all $s \in \mathbb{R}_{+}$, and $\tilde{F}$ is upper semicontinuous on $\tilde{\pi}\left(S, \mathbb{R}_{+}\right)$then $(X, \pi, M, I)$ is parallelizable.

Proof. According to Lemma 4.2.7, it is enough to prove that $h: X \rightarrow S \times \mathbb{R}_{+}$is continuous on $X \backslash M$. Let $x \in X \backslash M$ and $\left\{x_{n}\right\}_{n \in \mathbb{N}} \subset X$ be a sequence such that $x_{n} \stackrel{n \rightarrow \infty}{\longrightarrow} x$. We have some cases to consider.

Case 1: $x \in \tilde{F}(S,(0, \infty)) \backslash M$.

Let us show firstly that $\tau_{S}\left(x_{n}\right) \stackrel{n \rightarrow \infty}{\longrightarrow} \tau_{S}(x)$. Note that $\tilde{\pi}\left(x, \tau_{S}(x)\right) \in S$. Since $M \cap S=\emptyset$, it follows by Lemma 4.1.5 that there exists a sequence $\left\{\beta_{n}\right\}_{n \in \mathbb{N}}$ in $\mathbb{R}_{+}$such that $\beta_{n} \stackrel{n \rightarrow \infty}{\longrightarrow} 0$ and $\tilde{\pi}\left(x_{n}, \tau_{S}(x)+\beta_{n}\right) \stackrel{n \rightarrow \infty}{\longrightarrow} \tilde{\pi}\left(x, \tau_{S}(x)\right) \in S$. Consequently, from condition $(\mathrm{T})$, we have

$$
\tau_{S}\left(\tilde{\pi}\left(x_{n}, \tau_{S}(x)+\beta_{n}\right)\right) \stackrel{n \rightarrow \infty}{\longrightarrow} 0
$$

By Lemma 4.4.2, we may assume that $\left\{x_{n}\right\}_{n \in \mathbb{N}} \subset \tilde{F}(S,(0, \infty)) \backslash M$. By Lemma 4.2.5, cases $(a)$ and $(b)$, we get

$$
\tau_{S}\left(x_{n}\right)=\tau_{S}(x)+\beta_{n} \pm \tau_{S}\left(\tilde{\pi}\left(x_{n}, \tau_{S}(x)+\beta_{n}\right)\right) \stackrel{n \rightarrow \infty}{\longrightarrow} \tau_{S}(x)
$$

Now, let $z_{x} \in S$ be such that $\tilde{\pi}\left(x, \tau_{S}(x)\right)=z_{x}$. By hypothesis $S \bigcap I(M)=\emptyset$, consequently, $\tau_{S}(x)$ is a non-jump time of $x$. Thus, by Lemma 4.1.5, $\tilde{\pi}\left(x_{n}, \tau_{S}\left(x_{n}\right)\right) \stackrel{n \rightarrow \infty}{\longrightarrow} \tilde{\pi}\left(x, \tau_{S}(x)\right)$. Hence,

$$
h\left(x_{n}\right)=\left(\tilde{\pi}\left(x_{n}, \tau_{S}\left(x_{n}\right)\right), \tau_{S}\left(x_{n}\right)\right) \stackrel{n \rightarrow \infty}{\longrightarrow}\left(\tilde{\pi}\left(x, \tau_{S}(x)\right), \tau_{S}(x)\right)=h(x) .
$$


Case 2: $x \in \tilde{\pi}(S,(0, \infty)) \backslash M$.

As in the previous case, let us prove that $\tau_{S}\left(x_{n}\right) \stackrel{n \rightarrow \infty}{\longrightarrow} \tau_{S}(x)$. Let $z_{x} \in S$ be such that $x=$ $\tilde{\pi}\left(z_{x}, \tau_{S}(x)\right)$. Since $S$ is a section, $\tilde{F}\left(x, \tau_{S}(x)\right)=\left\{z_{x}\right\}$. By hypothesis, there exists $w_{n} \in \tilde{F}\left(x_{n}, \tau_{S}(x)\right)$ for all $n \in \mathbb{N}$. Since $d_{H}\left(\tilde{F}\left(x_{n}, \tau_{S}(x)\right), \tilde{F}\left(x, \tau_{S}(x)\right) \stackrel{n \rightarrow \infty}{\longrightarrow} 0\right.$, we obtain $w_{n} \stackrel{n \rightarrow \infty}{\longrightarrow} z_{x} \in S$. By condition (T), $\tau_{S}\left(w_{n}\right) \stackrel{n \rightarrow \infty}{\longrightarrow} 0$.

Since $X=\tilde{F}\left(S, \mathbb{R}_{+}\right) \cup \tilde{\pi}\left(S, \mathbb{R}_{+}\right)$, we can decompose $\left\{w_{n}\right\}_{n \in \mathbb{N}}$ into

$$
\left\{w_{n}\right\}_{n \in \mathbb{N}}=\left\{w_{m}\right\}_{m \in \mathbb{N}_{1}} \bigcup\left\{w_{\ell}\right\}_{\ell \in \mathbb{N}_{2}},
$$

where $\mathbb{N}=\mathbb{N}_{1} \cup \mathbb{N}_{2},\left\{w_{m}\right\}_{m \in \mathbb{N}_{1}} \subset \tilde{F}\left(S, \mathbb{R}_{+}\right)$and $\left\{w_{\ell}\right\}_{\ell \in \mathbb{N}_{2}} \subset \tilde{\pi}\left(S, \mathbb{R}_{+}\right)$.

If $\mathbb{N}_{1}$ is a countably infinite set, then it follows by Lemma 4.2 .5 , case $(b)$, that

$$
\tau_{S}\left(x_{m}\right)=\tau_{S}\left(\tilde{\pi}\left(w_{m}, \tau_{S}(x)\right)\right)=\tau_{S}(x)-\tau_{S}\left(w_{m}\right) \stackrel{m \rightarrow \infty}{\longrightarrow} \tau_{S}(x) .
$$

If $\mathbb{N}_{2}$ is a countably infinite set, then it follows by Lemma 4.2 .5 , case $(c)$, that

$$
\tau_{S}\left(x_{\ell}\right)=\tau_{S}\left(\tilde{\pi}\left(w_{\ell}, \tau_{S}(x)\right)\right)=\tau_{S}\left(w_{\ell}\right)+\tau_{S}(x) \stackrel{\ell \rightarrow \infty}{\longrightarrow} \tau_{S}(x)
$$

Consequently, $\tau_{S}\left(x_{n}\right) \stackrel{n \rightarrow \infty}{\longrightarrow} \tau_{S}(x)$.

Now, we claim that there exists $n_{0} \in \mathbb{N}$ such tha $x_{n} \in \tilde{\pi}(S,(0, \infty)) \backslash M$ for all $n \geqslant n_{0}$. In fact, suppose to the contrary that there exists a subsequence $\left\{x_{n_{k}}\right\}_{k \in \mathbb{N}} \subset \tilde{F}(S,(0, \infty))$. Thus, one can find $v_{k} \in S$ such that $\tilde{\pi}\left(x_{n_{k}}, \tau_{S}\left(x_{n_{k}}\right)\right)=v_{k}, k \in \mathbb{N}$. Since $w_{n_{k}} \in \tilde{F}\left(x_{n_{k}}, \tau_{S}(x)\right)$ for all $k \in \mathbb{N}$, we obtain $\tilde{\pi}\left(w_{n_{k}}, \tau_{S}(x)+\tau_{S}\left(x_{n_{k}}\right)\right)=v_{k}$, i.e., $w_{n_{k}} \in \tilde{F}\left(S, \mathbb{R}_{+}\right)$for all $k \in \mathbb{N}$. Using Lemma 4.2.5, case $(b)$, for $k$ sufficiently large, we get

$$
0=\tau_{S}\left(v_{k}\right)=\tau_{S}\left(\tilde{\pi}\left(w_{n_{k}}, \tau_{S}(x)+\tau_{S}\left(x_{n_{k}}\right)\right)\right)=\tau_{S}(x)+\tau_{S}\left(x_{n_{k}}\right)-\tau_{S}\left(w_{n_{k}}\right)
$$

As $k \rightarrow \infty$ in the above equality, we obtain $\tau_{S}(x)=0$ which is an absurd because $\tau_{S}(x)>0(x \notin S)$. Hence, there exists $n_{0} \in \mathbb{N}$ such tha $x_{n} \in \tilde{\pi}(S,(0, \infty)) \backslash M$ for all $n \geqslant n_{0}$. Consequently, there exists $z_{x_{n}} \in S$ such that $\tilde{\pi}\left(z_{x_{n}}, \tau_{S}\left(x_{n}\right)\right)=x_{n}$ for all $n \geqslant n_{0}$.

On the other hand, since $d_{H}\left(\tilde{F}\left(x_{n}, \tau_{S}\left(x_{n}\right)\right), \tilde{F}\left(x, \tau_{S}(x)\right) \stackrel{n \rightarrow \infty}{\longrightarrow} 0\right.$ and $F\left(x, \tau_{S}(x)\right)=\left\{z_{x}\right\}$, we conclude that $z_{x_{n}} \stackrel{n \rightarrow \infty}{\longrightarrow} z_{x}$. Hence,

$$
h\left(x_{n}\right)=\left(z_{x_{n}}, \tau_{S}\left(x_{n}\right)\right) \stackrel{n \rightarrow \infty}{\longrightarrow}\left(z_{x}, \tau_{S}(x)\right)=h(x) .
$$




\section{Case 2.3: $x \in S$.}

In this case, $\tau_{S}(x)=0$. If $\left\{x_{n}\right\}_{n \in \mathbb{N}}$ admits a subsequence $\left\{x_{n_{k}}\right\}_{k \in \mathbb{N}} \subset \tilde{F}\left(S, \mathbb{R}_{+}\right)$then using the fact that $\tau_{S}\left(x_{n_{k}}\right) \stackrel{k \rightarrow \infty}{\longrightarrow} 0$ and Lemma 4.1.4, we get

$$
h\left(x_{n_{k}}\right)=\left(\tilde{\pi}\left(x_{n_{k}}, \tau_{S}\left(x_{n_{k}}\right)\right), \tau_{S}\left(x_{n_{k}}\right)\right) \stackrel{k \rightarrow \infty}{\longrightarrow}(x, 0)=h(x) .
$$

On the other hand, if $\left\{x_{n}\right\}_{n \in \mathbb{N}}$ admits a subsequence $\left\{x_{n_{m}}\right\}_{m \in \mathbb{N}} \subset \tilde{\pi}\left(S, \mathbb{R}_{+}\right)$then there exists $\left\{z_{x_{n_{m}}}\right\}_{m \in \mathbb{N}} \subset S$ such that $\tilde{\pi}\left(z_{x_{n_{m}}}, \tau_{S}\left(x_{n_{m}}\right)\right)=x_{n_{m}}$ for all $m \in \mathbb{N}$. Since

$$
d_{H}\left(\tilde{F}\left(x_{n}, \tau_{S}\left(x_{n}\right)\right), \tilde{F}(x, 0)\right) \stackrel{n \rightarrow \infty}{\longrightarrow} 0
$$

and $\tilde{F}(x, 0)=\{x\}$, we conclude that $z_{x_{m}} \stackrel{m \rightarrow \infty}{\longrightarrow} x$. Thus,

$$
h\left(x_{n_{m}}\right)=\left(z_{x_{n_{m}}}, \tau_{S}\left(x_{n_{m}}\right)\right) \stackrel{m \rightarrow \infty}{\longrightarrow}(x, 0)=h(x) .
$$

Therefore, $h\left(x_{n}\right) \stackrel{n \rightarrow \infty}{\longrightarrow} h(x)$.

This concludes the proof and $(X, \pi, M, I)$ is parallelizable. 
CHAPTER

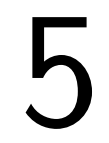

\section{PARALLELIZABILITY AND THE REGION OF WEAK ATTRACTION}

In this chapter, we present sufficient conditions for the region of weak attraction of a compact, strongly invariant and asymptotically stable set to be parallelizable. This study will be done for an impulsive semidynamical system $(X, \pi, M, I)$. When $M=\emptyset$, the results presented in this chapter still valid for semidynamical system without impulses.

The results of this chapter are presented in the paper (BONOTTO; PACIFICO, 2021a).

\subsection{Parallelizable impulsive region of weak attraction}

Let $(X, \pi, M, I)$ be an impulsive semidynamical system. Throughout this section, we shall assume the following general conditions:

H0) $X$ is locally compact.

H1) Any negative semisolution through $x \in X$ with respect to $\left(X, \pi, \mathbb{R}_{+}\right)$is defined on $I_{x}=$ $(-\infty, 0]$.

H2) The impulsive function $I: M \rightarrow N$ of the ISS $(X, \pi, M, I)$ is a homeomorphism with inverse $J: N \rightarrow M$, where $N=I(M)$.

H3) Each element of $M$ satisfies condition (C) and $I(M) \cap M=\emptyset$. 
We start by presenting an auxiliary result. The concept of an impulsive region of weak attraction is defined in Definition 2.2.11.

Proposition 5.1.1. Let $A \subset X$ be a closed asymptotically $\tilde{\pi}$-stable set. If $x \in \tilde{P}_{w}^{+}(A) \backslash M$ then $\tilde{J}^{+}(x) \subset A$.

Proof. Let $x \in \tilde{P}_{w}^{+}(A) \backslash M$ and $y \in \tilde{J}^{+}(x)$. Suppose to the contradiction that $y \notin A$. Set $\alpha=d(y, A)>$ 0 . Since $A$ is orbitally $\tilde{\pi}$-stable, there exists a positively $\tilde{\pi}$-invariant neighborhood $V_{\alpha}$ of $A$ such that $V_{\alpha} \subset B\left(A, \frac{\alpha}{2}\right)$. Consequently,

$$
\tilde{\pi}\left(V_{\alpha},[0, \infty)\right) \subset B\left(A, \frac{\alpha}{2}\right) .
$$

Since $x \in \tilde{P}_{w}^{+}(A) \backslash M$, one can find $T_{x}>0, T_{x} \neq \sum_{j=0}^{k} \phi\left(x_{j}^{+}\right)$for every $k \in \mathbb{N}$, such that $\tilde{\pi}\left(x, T_{x}\right) \in V_{\alpha}$. As $\pi$ is continuous on $X \times \mathbb{R}_{+}, I$ is continuous on $M$, and $\phi$ is continuous on $X \backslash M$ (we are assuming that condition $\mathrm{H} 3$ ) holds), we may obtain a neighborhood $V_{x}$ of $x$ such that

$$
\tilde{\pi}\left(V_{x}, T_{x}\right) \subset V_{\alpha}
$$

By (5.1.1) and (5.1.2), we conclude that

$$
\tilde{\pi}\left(V_{x}, T_{x}+t\right) \subset B\left(A, \frac{\alpha}{2}\right) \text { for all } t \geqslant 0 .
$$

On the other hand, since $y \in \tilde{J}^{+}(x)$, there are sequences $\left\{x_{n}\right\}_{n \in \mathbb{N}} \subset X$ and $\left\{t_{n}\right\}_{n \in \mathbb{N}} \subset \mathbb{R}_{+}$ such that $x_{n} \stackrel{n \rightarrow \infty}{\longrightarrow} x, t_{n} \stackrel{n \rightarrow \infty}{\longrightarrow} \infty$ and $\tilde{\pi}\left(x_{n}, t_{n}\right) \stackrel{n \rightarrow \infty}{\longrightarrow} y$. Thus, by (5.1.3), we get

$$
y=\lim _{n \rightarrow \infty} \tilde{\pi}\left(x_{n}, t_{n}\right) \in \overline{B\left(A, \frac{\alpha}{2}\right)}
$$

which is a contradiction. Therefore, $\tilde{J}^{+}(x) \subset A$.

In the next result, we present another auxiliary result concerning asymptotically $\tilde{\pi}$-stability.

Theorem 5.1.1. (BONOTTO; GRULHA JR, 2010, Theorem 3.14 ) Let $(X, \pi, M, I)$ be an ISS, $X$ be locally compact and $A \subset X$ be compact. The following conditions are equivalent:

(1) There exists a function $\psi: X \rightarrow \mathbb{R}_{+}$satisfying the following properties:

(i) $\psi$ is continuous in $X \backslash(M \backslash A)$. 
(ii) For every $\varepsilon>0$, there is a $\delta>0$ such that $\psi(x) \leqslant \varepsilon$ whenever $d(x, A) \leqslant \delta$.

(iii) For every $\varepsilon>0$, there is a $\delta>0$ such that $\psi(x) \geqslant \delta$ whenever $d(x, A) \geqslant \varepsilon$ and $x \notin M$.

(iv) $\psi(\tilde{\pi}(x, t)) \leqslant \psi(x)$ for every $x \in X \backslash M$ and $t \geqslant 0$.

(v) There is $\delta>0$ such that if $x \in B(A, \delta) \backslash A$ then $\psi(\tilde{\pi}(x, t)) \rightarrow 0$ when $t \rightarrow \infty$.

(2) $A$ is asymptotically $\tilde{\pi}$-stable.

In Theorem 5.1.2, we present a slightly modification in the statement of Theorem 5.1.1. More precisely, we added the condition that $\psi(\tilde{\pi}(x, t))<\psi(x)$ for all $t>0$, whenever $x \in$ $B(A, \delta) \backslash(A \cup M)$.

Theorem 5.1.2. Let $(X, \pi, M, I)$ be an ISS, $X$ be locally compact and $A \subset X$ be compact. The following conditions are equivalent:

(1) There exists a function $\psi: X \rightarrow \mathbb{R}_{+}$satisfying the following properties:

(i) $\psi$ is continuous in $X \backslash(M \backslash A)$.

(ii) For every $\varepsilon>0$, there is a $\delta>0$ such that $\psi(x) \leqslant \varepsilon$ whenever $d(x, A) \leqslant \delta$.

(iii) For every $\varepsilon>0$, there is a $\delta>0$ such that $\psi(x) \geqslant \delta$ whenever $d(x, A) \geqslant \varepsilon$ and $x \notin M$.

(iv) $\psi(\tilde{\pi}(x, t)) \leqslant \psi(x)$ for every $x \in X \backslash M$ and $t \geqslant 0$.

(v) There is $\delta>0$ such that if $x \in B(A, \delta) \backslash A$ then $\psi(\tilde{\pi}(x, t)) \rightarrow 0$ when $t \rightarrow \infty$. Further, if $x \in B(A, \delta) \backslash(A \cup M)$ then $\psi(\tilde{\pi}(x, t))<\psi(x)$ for all $t>0$.

(2) $A$ is asymptotically $\tilde{\pi}$-stable.

Proof. From Theorem 5.1.1, condition (1) implies condition (2). On the other hand, by Theorem 5.1.1, if condition (2) holds, then there exists a function $V: X \rightarrow \mathbb{R}_{+}$satisfying conditions $(i),(i i),(i i i)$ and $(i v)$. Moreover, there is $\delta>0$ such that if $x \in B(A, \delta) \backslash A$, then $V(\tilde{\pi}(x, t)) \rightarrow 0$ when $t \rightarrow \infty$.

Now, define the function $\psi: X \rightarrow \mathbb{R}_{+}$by

$$
\psi(x)=V(x)+\int_{0}^{\infty} V(\tilde{\pi}(x, t)) e^{-t} d t .
$$


This function satisfies conditions $(i),(i i),(i i i)$ and $(i v)$, and $\psi(\tilde{\pi}(x, t)) \rightarrow 0$ when $t \rightarrow \infty$ for all $x \in B(A, \delta) \backslash A$. In order to conclude that condition $(v)$ holds, suppose to the contradiction that there exist $x_{0} \in B(A, \delta) \backslash(A \cup M)$ and $\lambda>0$ such that $\psi\left(\tilde{\pi}\left(x_{0}, \lambda\right)\right)=\psi\left(x_{0}\right)$. Note that

$$
0=\psi\left(\tilde{\pi}\left(x_{0}, \lambda\right)\right)-\psi\left(x_{0}\right)=V\left(\tilde{\pi}\left(x_{0}, \lambda\right)\right)-V\left(x_{0}\right)+\int_{0}^{\infty}\left[V\left(\tilde{\pi}\left(x_{0}, \lambda+t\right)\right)-V\left(\tilde{\pi}\left(x_{0}, t\right)\right)\right] e^{-t} d t .
$$

Thus, using condition $(i v)$, we obtain

$$
0 \leqslant V\left(x_{0}\right)-V\left(\tilde{\pi}\left(x_{0}, \lambda\right)\right)=\int_{0}^{\infty}\left[V\left(\tilde{\pi}\left(x_{0}, \lambda+t\right)\right)-V\left(\tilde{\pi}\left(x_{0}, t\right)\right)\right] e^{-t} d t \leqslant 0 .
$$

Hence, $V\left(\tilde{\pi}\left(x_{0}, \lambda+t\right)\right)=V\left(\tilde{\pi}\left(x_{0}, t\right)\right)$ for all $t \geqslant 0$. Consequently, $V\left(\tilde{\pi}\left(x_{0}, k \lambda\right)\right)=V\left(x_{0}\right)$ for all $k \in \mathbb{N}$. Since $V\left(\tilde{\pi}\left(x_{0}, k \lambda\right)\right) \stackrel{k \rightarrow \infty}{\longrightarrow} 0$, we get $V\left(x_{0}\right)=0$ which is a contradiction as $x_{0} \notin A$. Therefore, $\psi(\tilde{\pi}(x, t))<\psi(x)$ for all $x \in B(A, \delta) \backslash(A \cup M)$ and all $t>0$, and the proof is complete.

Now, we present the main result of this section. This result involves the concept of property of negative unicity which was defined in Definition 3.4.1.

Theorem 5.1.3. Assume that conditions (H0)-(H3) hold. Let $(X, \pi, M, I)$ be an impulsive semidynamical system and $A \subset X$ be compact, strongly invariant and asymptotically $\tilde{\pi}$-stable. Assume that there exists $\gamma>0$ such that $\left(X, \pi, \mathbb{R}_{+}\right)$has the property of negative unicity on the set $B(A, \gamma) \backslash A$. If $B(A, \gamma) \cap M=\emptyset, A \cap I(M)=\emptyset$ and for every $x \in \tilde{P}_{w}^{+}(A) \backslash(A \cup M)$ one can find $k=k(x) \in \mathbb{N}_{0}$ such that $\phi\left(x_{k}^{+}\right)=\infty$, then $\left(\tilde{P}_{w}^{+}(A) \backslash(A \cup M), \pi, M, I\right)$ is parallelizable.

Proof. According to Theorem 4.3.2, we need to prove the existence of a compact section $S \subset$ $\tilde{P}_{w}^{+}(A) \backslash(A \cup M)$ with section function $\tau_{S}$ continuous on $\tilde{P}_{w}^{+}(A) \backslash(A \cup M)$. Note that $\tilde{P}_{w}^{+}(A) \backslash(A \cup M)$ is positively $\tilde{\pi}$-invariant since $A$ is strongly invariant and $I(M) \cap M=\emptyset$.

Since $A$ is asymptotically $\tilde{\pi}$-stable, by using Theorem 5.1.2, one can find a non-negative function $\psi$ defined on $X$ satisfying the conditions $(i),(i i),(i i i),(i v)$ and $(v)$ presented in Theorem 5.1.2.

By hypotheses, $A \cap I(M)=\emptyset, A$ is compact and $I(M)$ is closed. Thus there exists $\delta_{1}>0$ such that $I(M) \cap B\left(A, \delta_{1}\right)=\emptyset$. Further, by condition (iii) of $\psi$, there exists $\kappa>0$ such that $\psi(x) \geqslant \kappa$ for all $x \in I(M)$.

On the other hand, we have $A$ is compact and orbitally $\tilde{\pi}$-stable. By Theorem 2.2.1, $A$ is uniformly $\tilde{\pi}$-stable. Consider $\delta>0$ from condition $(v)$ of $\psi$. Then there exists $\delta_{0}>0, \delta_{0}<$ 
$\min \left\{\delta, \gamma, \delta_{1}\right\}$, such that $\overline{B\left(A, \delta_{0}\right)}$ is compact and $\tilde{\pi}^{+}\left(\overline{B\left(A, \delta_{0}\right)}\right) \subset B(A, \delta)$. Note that $\overline{B\left(A, \delta_{0}\right)} \subset$ $\tilde{P}_{w}^{+}(A)$. Now, define

$$
m_{0}=\inf \left\{\psi(x): x \in \partial B\left(A, \delta_{0}\right)\right\} .
$$

It follows by the property (iii) of $\psi$ that $m_{0}>0$. Also, by condition (iii), there exists $\beta>0$ such that

$$
\psi(y) \geqslant \beta \quad \text { whenever } \quad d(y, A) \geqslant \delta_{0} .
$$

Let $0<v<\min \left\{m_{0}, \kappa, \beta\right\}$. We claim that $S_{v}=\left\{x \in \overline{B\left(A, \delta_{0}\right)}: \psi(x)=v\right\}$ is a section for the ISS $\left(\tilde{P}_{w}^{+}(A) \backslash(A \cup M), \pi, M, I\right)$. Note that $S_{v}$ is compact in $\tilde{P}_{w}^{+}(A) \backslash(A \cup M)$. In order to prove the claim, we shall consider the following steps.

Step 1: Let $x \in \tilde{P}_{w}^{+}(A) \backslash(A \cup M)$. Then $x \in S_{v}$ if and only if $\psi(x)=v$.

In fact, suppose $\psi(x)=v$ and $x \notin \overline{B\left(A, \delta_{0}\right)}$. By hypothesis, there exists $k=k(x) \in \mathbb{N}_{0}$ such that $\phi\left(x_{k}^{+}\right)=\infty$. Since $x \in \tilde{P}_{w}^{+}(A) \backslash(A \cup M)$, there exists $T_{x}>0$ such that $\tilde{\pi}\left(x_{k}^{+}, T_{x}\right)=\pi\left(x_{k}^{+}, T_{x}\right) \in$ $B(A, \delta) \backslash A$. Consequently, by the property $(v)$ of $\psi$, we get $\psi\left(\tilde{\pi}\left(x_{k}^{+}, T_{x}+t\right)\right) \stackrel{t \rightarrow \infty}{\longrightarrow} 0$. Now, taking into account the condition (iii) of $\psi$, one can find $t_{x}^{1}>0$ such that $\tilde{\pi}\left(x_{k}^{+}, T_{x}+t_{x}^{1}\right) \in B\left(A, \delta_{0}\right) \backslash A$. As $\tilde{\pi}^{+}\left(x_{k}^{+}\right)=\pi^{+}\left(x_{k}^{+}\right)$, there exists $0 \leqslant t_{x}^{2} \leqslant t_{x}^{1}$ such that $\tilde{\pi}\left(x_{k}^{+}, T_{x}+t_{x}^{2}\right) \in \partial B\left(A, \delta_{0}\right)$. If $k=0$ (recall that $\left.x=x_{0}^{+}\right)$then

$$
m_{0} \leqslant \psi\left(\tilde{\pi}\left(x, T_{x}+t_{x}^{2}\right)\right) \leqslant \psi(x)=v<m_{0}
$$

which is a contradiction. And, if $k \geqslant 1$, then

$$
m_{0} \leqslant \psi\left(\tilde{\pi}\left(x_{k}^{+}, T_{x}+t_{x}^{2}\right)\right)=\psi\left(\tilde{\pi}\left(x, \sum_{j=0}^{k-1} \phi\left(x_{j}^{+}\right)+T_{x}+t_{x}^{2}\right)\right) \leqslant \psi(x)=v<m_{0}
$$

which is also a contradiction. Hence, $x \in \overline{B\left(A, \delta_{0}\right)}$ which implies $x \in S_{V}$.

Step 2: Let $x \in \tilde{P}_{w}^{+}(A) \backslash(A \cup M)$. Then $x \in \tilde{F}\left(S_{v},(0, \infty)\right)$ if and only if $\psi(x)>v$.

Indeed, assume at first that $x \in \tilde{F}\left(S_{v},(0, \infty)\right)$. Then there are $z_{x} \in S_{v}$ and $t_{x}>0$ such that $\tilde{\pi}\left(x, t_{x}\right)=z_{x}$. Since $S_{V} \cap I(M)=\emptyset\left(\delta_{0}<\delta_{1}\right)$, it follows that $t_{x}$ is a non-jump time (see Definition 2.2.7). Thus there exists $s_{x}<t_{x}$ such that $\tilde{\pi}\left(x, s_{x}\right) \in B(A, \delta) \backslash A$. According to conditions $(i v)$ and $(v)$ of $\psi$, we obtain

$$
v=\psi\left(z_{x}\right)=\psi\left(\tilde{\pi}\left(x, t_{x}\right)\right)=\psi\left(\tilde{\pi}\left(\tilde{\pi}\left(x, s_{x}\right), t_{x}-s_{x}\right)\right)<\psi\left(\tilde{\pi}\left(x, s_{x}\right)\right) \leqslant \psi(x) .
$$


Now, assume that $\psi(x)>v$. By hypothesis, there exists $k=k(x) \in \mathbb{N}_{0}$ such that $\phi\left(x_{k}^{+}\right)=\infty$. As in the proof of Step 1, we can obtain $T_{x}>0$ such that

$$
\tilde{\pi}\left(x_{k}^{+}, T_{x}\right)=\pi\left(x_{k}^{+}, T_{x}\right) \in B(A, \delta) \backslash A \quad \text { and } \quad \psi\left(\tilde{\pi}\left(x_{k}^{+}, T_{x}+t\right)\right) \stackrel{t \rightarrow \infty}{\longrightarrow} 0 .
$$

If $k=0$, then there exists $\bar{t}>0$ such that $\psi\left(\tilde{\pi}\left(x, T_{x}+\bar{t}\right)\right)=v$. By Step $1, \tilde{\pi}\left(x, T_{x}+\bar{t}\right) \in S_{v}$, i.e., $x \in \tilde{F}\left(S_{v},(0, \infty)\right)$.

If $k \geqslant 1$, then there is $T_{v}>T_{x}$ such that $\psi\left(\tilde{\pi}\left(x_{k}^{+}, T_{v}\right)\right)<v$. As $\tilde{\pi}^{+}\left(x_{k}^{+}\right)=\pi^{+}\left(x_{k}^{+}\right)$and $\psi\left(x_{k}^{+}\right) \geqslant \kappa>v$, there exists $s_{x}^{1} \in\left(0, T_{v}\right)$ such that $\psi\left(\tilde{\pi}\left(x_{k}^{+}, s_{x}^{1}\right)\right)=v$. By Step $1, \tilde{\pi}\left(x_{k}^{+}, s_{x}^{1}\right) \in S_{v}$. Hence, $\tilde{\pi}\left(x, \sum_{j=0}^{k-1} \phi\left(x_{j}^{+}\right)+s_{x}^{1}\right)=\tilde{\pi}\left(x_{k}^{+}, s_{x}^{1}\right) \in S_{v}$, i.e., $x \in \tilde{F}\left(S_{v},(0, \infty)\right)$.

Step 3: Let $x \in \tilde{P}_{w}^{+}(A) \backslash(A \cup M)$. Then $x \in \tilde{\pi}\left(S_{v},(0, \infty)\right)$ if and only if $\psi(x)<v$.

In fact, if $x \in \tilde{\pi}\left(S_{v},(0, \infty)\right)$ then $x=\tilde{\pi}\left(z_{x}, r_{x}\right)$ for some $z_{x} \in S_{v}$ and $r_{x}>0$. Since $S_{v} \subset$ $B(A, \delta) \backslash A$ and $S_{v} \cap M=\emptyset\left(\delta_{0}<\gamma\right)$, it follows by condition $(v)$ of $\psi$ that

$$
\psi(x)=\psi\left(\tilde{\pi}\left(z_{x}, r_{x}\right)\right)<\psi\left(z_{x}\right)=v .
$$

Now, suppose that $\psi(x)<v$. Since $v<\beta$ and (5.1.4) holds, we have $d(x, A)<\delta_{0}$. We claim that there is $y_{0} \in \tilde{F}(x,(0, \infty))$ such that $\psi\left(y_{0}\right)=v$. If it does not happen, then there exists an impulsive negative semisolution $\tilde{\sigma}_{x}$ through $x$ defined on $I_{x}=(-\infty, 0]$ such that $\psi(w)<v$ for all $w \in \tilde{\sigma}_{x}((-\infty, 0])$. In this case, $\tilde{\sigma}_{x}=\sigma_{x}$ as $\tilde{\sigma}_{x}((-\infty, 0]) \subset B\left(A, \delta_{0}\right)$ and $\delta_{0}<\min \left\{\gamma, \delta_{1}\right\}$. Since $\left\{\tilde{\sigma}_{x}(-n)\right\}_{n \in \mathbb{N}} \subset B\left(A, \delta_{0}\right)$, the sequence $\left\{\tilde{\sigma}_{x}(-n)\right\}_{n \in \mathbb{N}}$ admits a convergent subsequence (which we denote by the same), to some point $z \in \overline{B\left(A, \delta_{0}\right)} \subset \tilde{P}_{w}^{+}(A) \backslash M$. By Proposition 2.3.1, we have

$$
\tilde{\pi}\left(\tilde{\sigma}_{x}(-n), n\right)=\tilde{\sigma}_{x}(-n+n)=\tilde{\sigma}_{x}(0)=x
$$

for every $n \in \mathbb{N}$, which implies that $x \in \tilde{J}^{+}(z) \subset A$ (by Proposition 5.1.1) and it is a contradiction. Therefore, there is $y_{0} \in \tilde{F}(x,(0, \infty))$ with $\psi\left(y_{0}\right)=v$, i.e., $x \in \tilde{\pi}\left(S_{v},(0, \infty)\right)$.

Step 4: The sets $\tilde{F}\left(S_{v},(0, \infty)\right), S_{v}$ and $\tilde{\pi}\left(S_{v},(0, \infty)\right)$ are pairwise disjoint and

$$
\tilde{P}_{w}^{+}(A) \backslash(A \cup M)=\tilde{F}\left(S_{V},(0, \infty)\right) \cup S_{V} \cup \tilde{\pi}\left(S_{V},(0, \infty)\right) .
$$

In fact, it is a consequence of Steps 1, 2, 3.

Step 5: Let $x \in \tilde{P}_{w}^{+}(A) \backslash(A \cup M)$. Then either there is a unique $t_{x} \in \mathbb{R}_{+}$such that $\tilde{\pi}(x, t) \in S_{v}$ or there are unique $z_{x} \in S_{v}$ and $t_{x}>0$ such that $\tilde{\pi}\left(z_{x}, t_{x}\right)=x$. 
Indeed, assume at first that $x \in S_{v}$. If $\tilde{\pi}\left(x, r_{1}\right) \in S_{v}$ for some $r_{1}>0$ then, using condition $(v)$ of $\psi$, we get $v=\psi\left(\tilde{\pi}\left(x, r_{1}\right)\right)<\psi(x)=v$ which is an absurd. Analogously, if $\pi\left(z, r_{2}\right)=x$ for some $z \in S_{v}$ and $r_{2}>0$ then it leads to the contradiction $v=\psi(x)=\psi\left(\pi\left(z, r_{2}\right)\right)<\psi(z)=v$. Hence, $t_{x}=0$ is the unique time for which $\tilde{\pi}\left(x, t_{x}\right) \in S_{v}$.

Now, suppose that $x \in \tilde{F}\left(S_{v},(0, \infty)\right)$. If there are $t_{1}, t_{2} \in \mathbb{R}_{+}$, with $t_{1}<t_{2}$, such that $\tilde{\pi}\left(x, t_{1}\right), \tilde{\pi}\left(x, t_{2}\right) \in S_{V}$ then

$$
v=\psi\left(\tilde{\pi}\left(x, t_{2}\right)\right)=\psi\left(\tilde{\pi}\left(\tilde{\pi}\left(x, t_{1}\right), t_{2}-t_{1}\right)\right)<\psi\left(\tilde{\pi}\left(x, t_{1}\right)\right)=v
$$

which is a contradiction. Hence, there is a unique $t_{x} \in \mathbb{R}_{+}$such that $\tilde{\pi}\left(x, t_{x}\right) \in S_{v}$. On the other hand, if $\tilde{F}\left(x, \mathbb{R}_{+}\right) \cap S_{v} \neq \emptyset$ then there are $z \in S_{v}$ and $t_{z} \geqslant 0$ such that $\tilde{\pi}\left(z, t_{z}\right)=x$. Thus $\tilde{\pi}\left(z, t_{z}+t_{x}\right) \in S_{v}$ and $v=\psi\left(\tilde{\pi}\left(z, t_{z}+t_{x}\right)\right)<\psi(z)=v$, which is a contradiction. Hence, in this case, $\tilde{F}\left(x, \mathbb{R}_{+}\right) \cap S_{v}=\emptyset$.

Lastly, suppose $x \in \tilde{\pi}\left(S_{v},(0, \infty)\right)$. By Step 3, $\psi(x)<v$. Further, by the proof of Step 3, we conclude that there is $z_{x} \in \tilde{F}(x,(0, \infty)) \cap S_{v}$. Let $t_{x}>0$ be such that $\tilde{\pi}\left(z_{x}, t_{x}\right)=x$. The uniqueness of $z_{x}$ and $t_{x}$ is due to the fact that $\left(X, \pi, \mathbb{R}_{+}\right)$has the property of negative unicity on $B(A, \gamma) \backslash A$. In this case, it is not difficult to see that $\pi\left(x, \mathbb{R}_{+}\right) \cap S_{v}=\emptyset$.

According to the Steps 1, 2, 3, 4 and 5, we conclude that $S_{V}$ is a section for the ISS $\left(\tilde{P}_{w}^{+}(A) \backslash(A \cup M), \pi, M, I\right)$. Thus, we can consider the section function $\tau_{v}: \tilde{P}_{w}^{+}(A) \backslash(A \cup M) \rightarrow \mathbb{R}_{+}$ associated with the section $S_{v}$ as defined in (4.2.1).

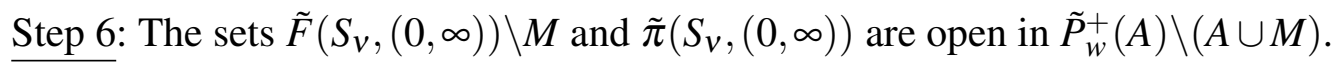

To conclude Step 6, it is enough to use Steps 2 and 3, and the continuity of $\psi$ on $X \backslash(M \backslash A)$. Note, by Lemma 4.2.3, that $M \subset \tilde{F}\left(S_{V},(0, \infty)\right)$.

In order to conclude this proof, we need to show that the section function $\tau_{S_{v}}$ is continuous on $\tilde{P}_{w}^{+}(A) \backslash(A \cup M)$. In fact, suppose to the contradiction that $\tau_{S_{v}}$ is discontinuous at some point $x \in \tilde{P}_{w}^{+}(A) \backslash(A \cup M)$. Then there are $\varepsilon>0$ and a sequence $\left\{x_{n}\right\}_{n \in \mathbb{N}} \subset \tilde{P}_{w}^{+}(A) \backslash(A \cup M)$ with $x_{n} \stackrel{n \rightarrow \infty}{\longrightarrow} x$ such that

$$
\left|\tau_{S_{v}}\left(x_{n}\right)-\tau_{S_{v}}(x)\right| \geqslant \varepsilon
$$

for all $n \in \mathbb{N}$. We have some cases to consider:

Case 1: $x \in \tilde{F}\left(S_{v},(0, \infty)\right) \backslash M$. 
By Step 6, we may assume that $\left\{x_{n}\right\}_{n \in \mathbb{N}} \subset \tilde{F}\left(S_{v},(0, \infty)\right) \backslash M$. Thus, there exist $z, z_{n} \in S_{v}$ such that $\tilde{\pi}\left(x, \tau_{S_{v}}(x)\right)=z$ and $\tilde{\pi}\left(x_{n}, \tau_{S_{v}}\left(x_{n}\right)\right)=z_{n}, n \in \mathbb{N}$. Using the compactness of $S_{v}$, we may assume without loss of generality that $\tilde{\pi}\left(x_{n}, \tau_{S_{v}}\left(x_{n}\right)\right) \stackrel{n \rightarrow \infty}{\longrightarrow} z_{0} \in S_{V}$. If $\tau_{S_{v}}\left(x_{n}\right) \stackrel{n \rightarrow \infty}{\longrightarrow} \infty$ (up to a subsequence), then $z_{0} \in \tilde{J}^{+}(x)$ which is a contradiction as $\tilde{J}^{+}(x) \subset A$ (see Proposition 5.1.1). Hence, we may assume that $\tau_{S_{v}}\left(x_{n}\right) \stackrel{n \rightarrow \infty}{\longrightarrow} t^{\prime}$.

We claim that $t^{\prime}$ is a non-jump time of $x$. In fact, otherwise, by Remark 4.1.1, there would exists a sequence $\left\{\beta_{n}\right\}_{n \in \mathbb{N}}$ in $\mathbb{R}$ such that $\beta_{n} \stackrel{n \rightarrow \infty}{\longrightarrow} 0, \tilde{\pi}\left(x_{n}, t^{\prime}+\beta_{n}\right) \in I(M)$ and $\tilde{\pi}\left(x_{n}, t^{\prime}+\beta_{n}\right) \stackrel{n \rightarrow \infty}{\longrightarrow}$ $\tilde{\pi}\left(x, t^{\prime}\right)$. Since $\psi\left(\tilde{\pi}\left(x_{n}, t^{\prime}+\beta_{n}\right)\right) \geqslant \kappa$ for every $n \in \mathbb{N}(\psi(w) \geqslant \kappa$ for all $w \in I(M)), \tilde{\pi}\left(x, t^{\prime}\right) \notin M$ and $\psi$ is continuous on $X \backslash(M \backslash A)$, we obtain the following estimate as $n \rightarrow \infty$,

$$
\psi\left(\tilde{\pi}\left(x, t^{\prime}\right)\right) \geqslant \kappa>v .
$$

On the other hand, by Lemma 4.1.5, there exists a sequence $\left\{\gamma_{n}\right\}_{n \in \mathbb{N}}$ in $\mathbb{R}_{+}$such that $\gamma_{n} \stackrel{n \rightarrow \infty}{\longrightarrow} 0$ and $\tilde{\pi}\left(x_{n}, \tau_{S_{v}}\left(x_{n}\right)+\gamma_{n}\right) \stackrel{n \rightarrow \infty}{\longrightarrow} \tilde{\pi}\left(x, t^{\prime}\right)$. Due to condition $(v)$ of $\psi$, we have

$$
\psi\left(\tilde{\pi}\left(x_{n}, \tau_{S_{v}}\left(x_{n}\right)+\gamma_{n}\right)\right)=\psi\left(\tilde{\pi}\left(z_{n}, \gamma_{n}\right)\right)<\psi\left(z_{n}\right)=v, \quad n \in \mathbb{N} .
$$

As $n \rightarrow \infty$ in the above inequality, we get

$$
\psi\left(\tilde{\pi}\left(x, t^{\prime}\right)\right) \leqslant v
$$

which contradicts (5.1.6). Hence, $t^{\prime}$ is a non-jump time of $x$. Consequently, by Remark 4.1.2,

$$
z_{n}=\tilde{\pi}\left(x_{n}, \tau_{S_{v}}\left(x_{n}\right)\right) \stackrel{n \rightarrow \infty}{\longrightarrow} \tilde{\pi}\left(x, t^{\prime}\right) .
$$

Since $\left\{z_{n}\right\}_{n \in \mathbb{N}} \subset S_{v}$, we conclude that $\tilde{\pi}\left(x, t^{\prime}\right) \in S_{v}$. By uniqueness of $\tau_{S_{v}}(x), \tau_{S_{v}}(x)=t^{\prime}$. Therefore, $\tau_{S_{V}}\left(x_{n}\right) \stackrel{n \rightarrow \infty}{\longrightarrow} \tau_{S_{v}}(x)$ and this contradicts (5.1.5).

Case 2: $x \in \tilde{\pi}\left(S_{v},(0, \infty)\right)$.

By Step 6, we may assume that $\left\{x_{n}\right\}_{n \in \mathbb{N}} \subset \tilde{\pi}\left(S_{v},(0, \infty)\right)$. Note that, there are $z_{n}, z \in S_{v}$ such that $\tilde{\pi}\left(z, \tau_{S_{v}}(x)\right)=x$ and $\tilde{\pi}\left(z_{n}, \tau_{S_{v}}\left(x_{n}\right)\right)=x_{n}, n \in \mathbb{N}$. By compactness of $S_{v}$, up to a subsequence, we have $z_{n} \stackrel{n \rightarrow \infty}{\longrightarrow} z^{\prime} \in S_{v}$. We may assume without loss of generality that $\tau_{S_{v}}\left(x_{n}\right) \stackrel{n \rightarrow \infty}{\longrightarrow} t^{\prime}$ (otherwise, we would have $\left.x \in \tilde{J}^{+}\left(z^{\prime}\right) \subset A\right)$.

If $t^{\prime}$ is a jump time of $z^{\prime}$, then by proceeding as in the proof of Case 1 , one can find a sequence $\left\{\beta_{n}\right\}_{n \in \mathbb{N}}$ in $\mathbb{R}$ such that $\beta_{n} \stackrel{n \rightarrow \infty}{\longrightarrow} 0, \tilde{\pi}\left(z_{n}, \tau_{S_{v}}\left(x_{n}\right)+\beta_{n}\right) \in I(M)$ and $\tilde{\pi}\left(z_{n}, \tau_{S_{v}}\left(x_{n}\right)+\beta_{n}\right) \stackrel{n \rightarrow \infty}{\longrightarrow} \tilde{\pi}\left(z^{\prime}, t^{\prime}\right)$. Since $\psi(w) \geqslant \kappa$ for all $w \in I(M)$, we get

$$
v<\kappa \leqslant \psi\left(\tilde{\pi}\left(z_{n}, \tau_{S_{v}}\left(x_{n}\right)+\beta_{n}\right)\right)<\psi\left(z_{n}\right)=v
$$


which is a contradiction. Hence, $t^{\prime}$ is a non-jump time of $z^{\prime}$. Thus, by Remark 4.1.2,

$$
x_{n}=\tilde{\pi}\left(z_{n}, \tau_{S_{v}}\left(x_{n}\right)\right) \stackrel{n \rightarrow \infty}{\longrightarrow} \tilde{\pi}\left(z^{\prime}, t^{\prime}\right) .
$$

Since $x_{n} \stackrel{n \rightarrow \infty}{\longrightarrow} x$, we get $\tilde{\pi}\left(z^{\prime}, t^{\prime}\right)=x=\tilde{\pi}\left(z, \tau_{S_{v}}(x)\right)$ which implies $z=z^{\prime}$ and $\tau_{S_{v}}(x)=t^{\prime}$ as $S_{V}$ is a section. Therefore, $\tau_{S_{v}}\left(x_{n}\right) \stackrel{n \rightarrow \infty}{\longrightarrow} \tau_{S_{v}}(x)$ contradicting (5.1.5).

Case 3: $x \in S_{V}$.

If $\left\{x_{n}\right\}_{n \in \mathbb{N}}$ admits a subsequence $\left\{x_{n_{k}}\right\}_{k \in \mathbb{N}} \subset \tilde{F}\left(S_{v},(0, \infty)\right)\left(\left\{x_{n_{k}}\right\}_{k \in \mathbb{N}} \subset \tilde{\pi}\left(S_{v},(0, \infty)\right)\right)$, then using the proof of Case 1 (Case 2), up to a subsequence, we obtain

$$
\tau_{S_{v}}\left(x_{n_{k}}\right) \stackrel{k \rightarrow \infty}{\longrightarrow} 0=\tau_{S_{v}}(x)
$$

contradicting (5.1.5). Analogously, if $\left\{x_{n}\right\}_{n \in \mathbb{N}}$ admits a subsequence $\left\{x_{n_{k}}\right\}_{k \in \mathbb{N}} \subset S_{v}$, then $\tau_{S_{v}}\left(x_{n_{k}}\right)=$ $0=\tau_{S_{v}}(x), k \in \mathbb{N}$, a contradiction.

Therefore, $\tau_{S_{v}}$ is continuous on $\tilde{P}_{w}^{+}(A) \backslash(A \cup M)$. Consequently, by Theorem 4.3.2, the ISS $\left(\tilde{P}_{w}^{+}(A) \backslash(A \cup M), \pi, M, I\right)$ is parallelizable.

If the impulsive set $M=\emptyset$ then we can obtain the following result on semidynamical systems without impulses.

Corollary 5.1.1. Let $\left(X, \pi, \mathbb{R}_{+}\right)$be a semidynamical system with $X$ locally compact. Let $A \subset X$ be compact, strongly invariant and asymptotically $\pi$-stable. Assume that there exists $\gamma>0$ such that $\left(X, \pi, \mathbb{R}_{+}\right)$has the property of negative unicity on the set $B(A, \gamma) \backslash A$. Then $\left(P_{w}^{+}(A) \backslash A, \pi, \mathbb{R}_{+}\right)$ is parallelizable.

\subsection{Application in autonomous differential equations}

Let $\|\cdot\|$ be a norm in $\mathbb{R}^{n}$ and $d$ be an induced metric. Let $f: \mathbb{R}^{n} \rightarrow \mathbb{R}^{n}$ be a function satisfying sufficient conditions such that the ordinary differential equation (ODE)

$$
x^{\prime}=f(x)
$$

defines a semidynamical system $\left(\mathbb{R}^{n}, \pi, \mathbb{R}_{+}\right)$on $\mathbb{R}^{n}$, where $\pi\left(x_{0}, t\right)=x\left(t, x_{0}\right)$ for all $x_{0} \in \mathbb{R}^{n}$ and all $t \in \mathbb{R}_{+}\left(x\left(t, x_{0}\right)\right.$ denotes a solution of (5.2.1) passing through the point $x_{0}$ at $\left.t=0\right)$. Now, let 
$M \subset \mathbb{R}^{n}$ be a hypersurface in $\mathbb{R}^{n}$ of class $C^{k}, k \geqslant 1$, satisfying the transversality condition: for each $p \in M$ we have $\left\langle\vec{n}_{p}, f(p)\right\rangle \neq 0$, where $\vec{n}_{p}$ denotes the normal vector of $M$ at $p$. Now, let $I: M \rightarrow \mathbb{R}^{n}$ be a continuous function such that $I(M) \cap M=\emptyset$. Then,

$$
\left(\mathbb{R}^{n}, \pi, M, I\right)
$$

defines an impulsive semidynamical system on $\mathbb{R}^{n}$ associated do the impulsive ordinary differential equation

$$
\left\{\begin{array}{l}
x^{\prime}=f(x), \\
I: M \rightarrow \mathbb{R}^{n} .
\end{array}\right.
$$

According to (BONOTTO et al., 2016), the impulsive set $M$ satisfies the condition STC. By Lemma 4.1.1, $M$ satisfies condition (C).

In the next definition, we characterize the concepts of parallelizability and dispersiveness for impulsive ODEs.

Definition 5.2.1. The impulsive ordinary differential equation (5.2.3) is called parallelizable (dispersive) on a strongly invariant subset $\mathscr{A} \subset \mathbb{R}^{n}$, when its associated impulsive semidynamical system on $\mathscr{A}$, that is, $(\mathscr{A}, \pi, M, I)$, is parallelizable (dispersive).

In what follows, we prove the absence of bounded solutions of the system (5.2.3) with closure contained in a parallelizable region.

Proposition 5.2.1. Let $A \subset \mathbb{R}^{n}$ be closed and strongly invariant with respect to the ISS (5.2.2). If the impulsive ODE (5.2.3) is parallelizable on $\mathbb{R}^{n} \backslash A$, then it does not admit any bounded solution $x$ such that

$$
\overline{\{x(t): t \geqslant 0\}} \subset \mathbb{R}^{n} \backslash A .
$$

Proof. Suppose to the contrary that there exist $x_{0} \in \mathbb{R}^{n} \backslash A$ and a bounded solution $x$ of (5.2.3) passing through the point $x_{0}$ at $t=0$ such that $\overline{\left\{x\left(t, x_{0}\right): t \geqslant 0\right\}} \subset \mathbb{R}^{n} \backslash A$. Let $\left\{t_{k}\right\}_{k \in \mathbb{N}} \subset \mathbb{R}_{+}$be a sequence such that $t_{k} \stackrel{k \rightarrow \infty}{\longrightarrow} \infty$ and consider the associated impulsive semidynamical system $\left(\mathbb{R}^{n} \backslash A, \pi, M, I\right)$. Thus, the sequence $\left\{\tilde{\pi}\left(x_{0}, t_{k}\right)\right\}_{k \in \mathbb{N}}$ admits a convergent subsequence with limit in $\mathbb{R}^{n} \backslash A$ and it implies that $\tilde{J}^{+}\left(x_{0}\right) \cap\left(\mathbb{R}^{n} \backslash A\right) \neq \emptyset$. Hence, $\left(\mathbb{R}^{n} \backslash A, \pi, M, I\right)$ is not dispersive. Consequently, by Theorem 4.4.1, $\left(\mathbb{R}^{n} \backslash A, \pi, M, I\right)$ is not parallelizable and it contradicts the hypothesis. Therefore, the result follows. 
Corollary 5.2.1. If the impulsive ODE (5.2.3) is parallelizable on $\mathbb{R}^{n}$, then it does not admit any bounded solution $x:[0, \infty) \rightarrow \mathbb{R}^{n}$.

If $M=\emptyset$, then the previous results are valid for continuous semidynamical systems.

Corollary 5.2.2. Assume that $M=\emptyset$. Let $A \subset \mathbb{R}^{n}$ be closed and strongly invariant subset with respect to $\left(\mathbb{R}^{n}, \pi, \mathbb{R}_{+}\right)$. If the ODE (5.2.1) is parallelizable on $\mathbb{R}^{n} \backslash A$, then it does not admit any bounded solution $x$ such that

$$
\overline{\{x(t): t \geqslant 0\}} \subset \mathbb{R}^{n} \backslash A .
$$

Corollary 5.2.3. Assume that $M=\emptyset$. If the ODE (5.2.1) is parallelizable on $\mathbb{R}^{n}$, then it does not admit any bounded solution $x:[0, \infty) \rightarrow \mathbb{R}^{n}$.

In the next proposition, we show that it is possible to obtain a function $V: \mathbb{R}^{n} \rightarrow \mathbb{R}$ whose derivative changes sign in the sets $\tilde{F}\left(S, \mathbb{R}_{+}\right)$and $\tilde{\pi}\left(S, \mathbb{R}_{+}\right)$, provided $S$ is a section.

Proposition 5.2.2. Assume that the impulsive ODE (5.2.3) is parallelizable on $\mathbb{R}^{n}$ with base $S$. Then there exists a continuous function $V: \mathbb{R}^{n} \rightarrow \mathbb{R}$ satisfying the conditions:

a) $\langle\nabla V(x), f(x)\rangle \leqslant 0$ if $x \in \tilde{F}\left(S, \mathbb{R}_{+}\right)$;

b) $\langle\nabla V(x), f(x)\rangle \geqslant 0$ if $x \in \tilde{\pi}\left(S, \mathbb{R}_{+}\right)$.

Proof. By Theorem 4.3.1, $S$ is a section with section function $\tau_{S}$ continuous on $\mathbb{R}^{n} \backslash M$. Define $V: \mathbb{R}^{n} \rightarrow \mathbb{R}$ by $V(x)=\tau_{S}(x)$ for each $x \in \mathbb{R}^{n}$. If $x \in \tilde{F}\left(S, \mathbb{R}_{+}\right)$, then it follows by Lemma 4.2.5 that $\tau_{S}(x)-\tau_{S}(\tilde{\pi}(x, t))>0$ for all $t \in\left[0, \tau_{S}(x)\right)$. Hence, $\langle\nabla V(x), f(x)\rangle \leqslant 0$. On the other hand, if $x \in$ $\tilde{\pi}\left(S, \mathbb{R}_{+}\right)$, then by Lemma 4.2.5, $\tau_{S}(\tilde{\pi}(x, t))-\tau_{S}(x)>0$ for all $t>0$. Thus, $\langle\nabla V(x), f(x)\rangle \geqslant 0$.

Corollary 5.2.4. Assume that $M=\emptyset$ and the ODE (5.2.1) is parallelizable on $\mathbb{R}^{n}$ with base $S$. Then there exists a continuous function $V: \mathbb{R}^{n} \rightarrow \mathbb{R}$ satisfying the conditions:

a) $\langle\nabla V(x), f(x)\rangle \leqslant 0$ if $x \in F\left(S, \mathbb{R}_{+}\right)$;

b) $\langle\nabla V(x), f(x)\rangle \geqslant 0$ if $x \in \pi\left(S, \mathbb{R}_{+}\right)$.

Let $A \subset \mathbb{R}^{n}$. Next, we establish sufficient conditions for the impulsive ODE (5.2.3) to be parallelizable on $\mathbb{R}^{n} \backslash A$. 
Theorem 5.2.1. Assume that conditions (H1)-(H2) presented in Section 5.1 hold. Let $A \subset \mathbb{R}^{n}$ be a compact strongly invariant set with respect to the impulsive ODE (5.2.3). Assume that there exists $\gamma>0$ such that $\left(\mathbb{R}^{n}, \pi, \mathbb{R}_{+}\right)$has the property of negative unicity on the set $B(A, \gamma) \backslash A$ and $B(A, \gamma) \cap M=\emptyset$. Further, assume there exists a function $V \in C^{1}\left(\mathbb{R}^{n}, \mathbb{R}_{+}\right)$satisfying the following conditions:

(a) $V(x)=0$ if $x \in A$, and for every sequence $\left\{x_{k}\right\}_{k \in \mathbb{N}} \subset \mathbb{R}^{n} \backslash A$ such that $V\left(x_{k}\right) \stackrel{k \rightarrow \infty}{\longrightarrow} 0$ we have $d\left(x_{k}, A\right) \stackrel{k \rightarrow \infty}{\longrightarrow} 0$

(b) $\nabla V(x) \cdot f(x) \leqslant-\alpha V(x)$ for all $x \in \mathbb{R}^{n} \backslash A$, where $\alpha>0$;

(c) $V(I(x))<V(x)$ for all $x \in M$.

Then the impulsive ODE (5.2.3) is parallelizable on $\mathbb{R}^{n} \backslash(A \cup M)$.

Proof. At first, we claim that $A$ is asymptotically $\tilde{\pi}$-stable. For that, we are going to show that the conditions of Theorem 5.1.2 are satisfied. Indeed, by hypothesis $V$ is continuous on $\mathbb{R}^{n}$. Let $\varepsilon>0$ be given. By the first part of condition $(a)$, there exists $\delta>0$ such that if $d(x, A) \leqslant \delta$ then $V(x) \leqslant \varepsilon$.

Now, let $\varepsilon>0$ be given and suppose that for every $k \in \mathbb{N}$ there exists $x_{k} \in \mathbb{R}^{n} \backslash B(A, \varepsilon)$ such that $V\left(x_{k}\right)<\frac{1}{k}$. But this contradicts the second part of condition $(a)$. Hence, there is $\delta>0$ such that $V(x) \geqslant \delta$ whenever $d(x, A) \geqslant \varepsilon$.

Let $x \in \mathbb{R}^{n} \backslash M$. If $x \in A$ then $V(\tilde{\pi}(x, t))=0=V(x)$ for all $t \geqslant 0$, since $A$ is strongly invariant. But, if $x \notin A$ then

$$
\frac{d}{d t} V(\pi(x, t))=\nabla V(\pi(x, t)) f(\pi(x, t)) \leqslant-\alpha V(\pi(x, t))
$$

for all $t \geqslant 0$. Consequently,

$$
V(\pi(x, t)) \leqslant e^{-\alpha t} V(x)<V(x)
$$

for all $t>0$. Now, using the fact that $V(I(z))<V(z)$ whenever $z \in M$, we conclude that $V(\tilde{\pi}(x, t)) \leqslant$ $V(x)$ for every $t \geqslant 0$. In conclusion, $V(\tilde{\pi}(x, t)) \leqslant V(x)$ for all $x \in \mathbb{R}^{n} \backslash M$ and $t \geqslant 0$.

By (5.2.4), if $x \in \mathbb{R}^{n} \backslash(A \cup M)$ then $V(\pi(x, t))<V(x)$ for all $t>0$, and $V(\tilde{\pi}(x, t)) \rightarrow 0$ when $t \rightarrow \infty$.

Hence, by Theorem 5.1.2, $A$ is asymptotically $\tilde{\pi}$-stable. Further, by (5.2.4), $\tilde{P}_{w}^{+}(A)=\mathbb{R}^{n}$. 
Since $A$ is strongly invariant and $M \cap B(A, \gamma)=\emptyset$ then $I(M) \cap A=\emptyset$.

Lastly, let $x \in \mathbb{R}^{n} \backslash(A \cup M)$. Since $A$ is asymptotically $\tilde{\pi}$-stable, there exists $t_{x} \geqslant 0$ such that $\tilde{\pi}(x, t) \in B(A, \gamma)$ for all $t \geqslant t_{x}$. But as $B(A, \gamma) \cap M=\emptyset$, one can find $k \in \mathbb{N}_{0}$ such that $\phi\left(x_{k}^{+}\right)=\infty$.

In conclusion, according to Theorem 5.1.3, the impulsive ODE (5.2.3) is parallelizable on $\mathbb{R}^{n} \backslash(A \cup M)$.

Finally, if $M=\emptyset$ then Theorem 5.2.1 holds for continuous semidynamical systems.

Corollary 5.2.5. Assume that $M=\emptyset$ and condition (H1) presented in Section 5.1 holds. Let $A \subset \mathbb{R}^{n}$ be a compact strongly invariant set with respect to the ODE (5.2.1). Assume that there exists $\gamma>0$ such that $\left(\mathbb{R}^{n}, \pi, \mathbb{R}_{+}\right)$has the property of negative unicity on the set $B(A, \gamma) \backslash A$. Further, assume there exists a function $V \in C^{1}\left(\mathbb{R}^{n}, \mathbb{R}_{+}\right)$satisfying the following conditions:

(a) $V(x)=0$ if $x \in A$, and for every sequence $\left\{x_{k}\right\}_{k \in \mathbb{N}} \subset \mathbb{R}^{n} \backslash A$ such that $V\left(x_{k}\right) \stackrel{k \rightarrow \infty}{\longrightarrow} 0$ we have $d\left(x_{k}, A\right) \stackrel{k \rightarrow \infty}{\longrightarrow} 0$

(b) $\nabla V(x) \cdot f(x) \leqslant-\alpha V(x)$ for all $x \in \mathbb{R}^{n} \backslash A$, where $\alpha>0$.

Then the ODE (5.2.1) is parallelizable on $\mathbb{R}^{n} \backslash A$. 

AFONSO, S. M.; BONOTTO, E. M.; JIMENEZ, M. Z. Negative trajectories in impulsive semidynamical systems. Journal of Differential Equations, v. 259, p. 964-988, 03 2015. Citations on pages $18,21,27,36,39$, and 40 .

ANTOSIEWICZ, H. A.; DUGUNDJI, J. Parallelizable flows and lyapunov's second method. Boletín de la Sociedad Matemática Mexicana. Segunda Serie, v. 5, 01 1960. Citations on pages 17,41 , and 56 .

BHATIA, N. P.; HAJEK, O. Local Semi-Dynamical Systems. Springer Berlin Heidelberg, 1969. (Lecture Notes in Mathematics). ISBN 9783540360995. Available: <https://books.google.com.br/ books?id=oit8CwAAQBAJ>. Citations on pages 21, 22, 24, 25, and 36.

BHATIA, N. P.; SZEGÖ, G. P. Stability Theory of Dynamical Systems. Springer Berlin Heidelberg, 2002. (Classics in Mathematics). ISBN 9783540427483. Available: <https://books.google. com.br/books?id=wP5dwTS6jg0C $>$. Citations on pages 17, 41, 57, and 74.

BISTROŃ, A. On minimal and invariant sets in semidynamical systems. Univ. Iagel. Acta Math., n. 41, p. 189-204, 2003. ISSN 0083-4386. Citations on pages 21 and 36.

. The semidynamical system near a closed negatively strongly invariant set. Univ. Iagel. Acta Math., n. 43, p. 109-124, 2005. Citations on pages 21 and 36.

BONOTTO, E. M. Flows of characteristic 0+ in impulsive semidynamical systems. Journal of Mathematical Analysis and Applications, v. 332, p. 81-96, 08 2007. Citations on pages 18, 21, 27 , and 29.

BONOTTO, E. M.; BORTOLAN, M. C.; CARABALLO, T.; COLLEGARI, R. Impulsive surfaces on dynamical systems. Acta Mathematica Hungarica, v. 150, p. 209-216, 06 2016. Citations on pages 27 and 94.

BONOTTO, E. M.; BORTOLAN, M. C.; CARVALHO, A. N.; CZAJA, R. Global attractors for impulsive dynamical systems - a precompact approach. Journal of Differential Equations, v. 259, p. 2602-2625, 04 2015. Citations on pages 18, 21, 27, 30, 34, 66, and 67.

BONOTTO, E. M.; FEDERSON, M. Topological conjugation and asymptotic stability in impulsive semidynamical systems. Journal of Mathematical Analysis and Applications, v. 326, p. 869881, 02 2007. Citations on pages 18, 21, 27, and 31. 
BONOTTO, E. M.; GIMENES, L. P.; SOUTO, G. M. Asymptotically almost periodic motions in impulsive semidynamical systems. Topological Methods in Nonlinear Analysis, v. 49, p. 133163, 2017. Citation on page 34.

BONOTTO, E. M.; GRULHA JR, N. G. Lyapunov stability of closed sets in impulsive semidynamical systems. Electronic Journal of Differential Equations, v. 2010, p. 1-18, 06 2010. Citations on pages $18,27,32$, and 86 .

BONOTTO, E. M.; KALITA, P. On attractors of generalized semiflows with impulses. J. Geom. Anal., v. 30, n. 2, p. 1412-1449, 2020. ISSN 1050-6926. Available: <https://doi.org/10.1007/ s12220-019-00143-0>. Citation on page 27.

BONOTTO, E. M.; MESQUITA, J.; PARREIRA, R. Global mild solutions for a nonautonomous $2 \mathrm{~d}$ navier-stokes equations with impulses at variable times. Journal of Mathematical Fluid Mechanics, v. 20, p. 801-818, 06 2018. Citation on page 27.

BONOTTO, E. M.; PACIFICO, T. A. Parallelizable impulsive semidynamical systems. Pre-print, 2021. Citations on pages $11,13,61$, and 85 .

Recursive properties on semidynamical systems: parallelizability and dispersiveness. Preprint, 2021. Citations on pages 11, 13, and 41.

BONOTTO, E. M.; SOUTO, G. M. On the lyapunov stability theory for impulsive dynamical systems. Topological Methods in Nonlinear Analysis, v. 53, p. 127-150, 02 2019. Citation on page 27.

CHURCH, K. E. M.; LIU, X. Cost-effective robust stabilization and bifurcation suppression. SIAM Journal on Control and Optimization, v. 57, p. 2240-2268, 01 2019. Citation on page 18.

CIESIELSKI, K. On semicontinuity in impulsive dynamical systems. Bulletin of The Polish Academy of Sciences Mathematics, v. 52, p. 71-80, 01 2004. Citations on pages 18, 21, 27, 32, 33 , and 34 .

On stability in impulsive dynamical systems. Bulletin of the Polish Academy of Sciences Mathematics, v. 52, p. 81-91, 01 2004. Citations on pages 18 and 27.

DASHKOVSKIY, S.; FEKETA, P.; KAPUSTYAN, O.; ROMANIUK, I. Invariance and stability of global attractors for multi-valued impulsive dynamical systems. Journal of Mathematical Analysis and Applications, v. 458, p. 193-218, 09 2018. Citation on page 18.

DING, B.; DING, C. Generalized recurrence in impulsive semidynamical systems. Topological Methods in Nonlinear Analysis, v. 50, p. 217-229, 08 2017. Citations on pages 18 and 27.

DING, C. Limit sets in impulsive semidynamical systems. Topological Methods in Nonlinear Analysis, v. 43, p. 97-115, 03 2014. Citations on pages 18 and 27. 
DING, C.; DUAN, Z.; PAN, S. Bendixson criterion in impulsive systems. Mathematical Methods in the Applied Sciences, v. 43, p. 1176-1182, 11 2020. Citations on pages 18 and 27.

EGAWA, J. Global parallelizability of local dynamical systems. Mathematical Systems Theory, v. 6, p. 133-144, 03 1972. Citation on page 56.

GUO, Y.; SHEN, Y.; GUI, W. Asymptotical stability of logic dynamical systems with random impulsive disturbances. IEEE Transactions on Automatic Control, v. 66, p. 513-525, 042021. Citation on page 18.

HáJEK, O. Parallelizability revisited. Proceedings of the American Mathematical Society, v. 27, p. 77-84, 01 1971. Citations on pages 41 and 56.

KAPUSTYAN, O. V.; PERESTYUK, M. O. Existence of global attractors for impulsive dynamical systems. Reports of the National Academy of Sciences of Ukraine, v. 12, p. 13-18, 122015. Citations on pages 18 and 27.

Global attractors in impulsive infinite-dimensional systems. Ukrainian Mathematical Journal, v. 68, p. 517-528, 11 2016. Citations on pages 18 and 27.

KAUL, S. On impulsive semidynamical systems ii. Journal of Mathematical Analysis and Applications, v. 150, p. 120-128, 07 1990. Citations on pages 18, 21, and 27.

. On impulsive semidynamical systems-ii. recursive properties. Nonlinear Analysis-theory Methods and Applications, v. 16, p. 635-645, 12 1991. Citations on pages 18 and 27.

LUO, D.; WANG, Q.-R. Dynamic analysis on an almost periodic predator-prey system with impulsive effects and time delays. Discrete \& Continuous Dynamical Systems - B, v. 26, p. 3427-3453, 01 2021. Citation on page 18.

MCCANN, R. C. Negative escape time in semidynamical systems. Funkcialaj Ekvacioj. Serio Internacia, v. 20, p. 39-47, 01 1977. Citation on page 35.

NEMYTSKII, V. V.; STEPANOV, V. V. Qualitative Theory of Differential Equations. Dover Publications, 1989. (Dover books on advanced mathematics). ISBN 9780486659541. Available: $<$ https://books.google.com.br/books?id=3-mpZDFifoYC>. Citation on page 17.

PACIFICO, T.; SOUZA, J.; TOZATTI, H. A note on parallelizable dynamical systems. Electronic Research Announcements in Mathematical Sciences, v. 24, p. 64-67, 06 2017. Citation on page 17 .

ROZKO, V. F. A certain class of almost periodic motions in systems with pulses. Diff. Uravm., v. 8, p. 2012-2022, 1972. Citation on page 26.

Ljapunov stability in discontinuous dynamic systems. Diff. Uravm., v. 11, p. 1005-1012, 1975. Citation on page 26. 
SUGIE, J.; ISHIHARA, Y. Attraction region for the classical lotka-volterra predator-prey model caused by impulsive effects. Qualitative Theory of Dynamical Systems, v. 20, p. paper no. 46, 26pp, 07 2021. Citation on page 18.

URA, T.; EGAWA, J. Isomorphism and parallelizability in dynamical systems theory. Mathematical Systems Theory, v. 7, p. 258-264, 09 1973. Citation on page 17.

VADIVOO, B. S.; CAO, J.; RAJA, R.; RAJCHAKIT, G.; SEADAWY, A. Controllability criteria of fractional differential dynamical systems with non-instantaneous impulses. IMA Journal of Mathematical Control and Information, v. 37, p. 777-793, 10 2020. Citation on page 18.

WHITNEY, H. Regular families of curves: I. Proceedings of the National Academy of Sciences of the United States of America, v. 18, p. 275-278, 04 1932. Citation on page 17.

YU , J.; TANG, X. Global attractivity in a delay population model under impulsive perturbations. Bulletin of the London Mathematical Society, v. 34, p. 319 - 328, 05 2002. Citation on page 18. 


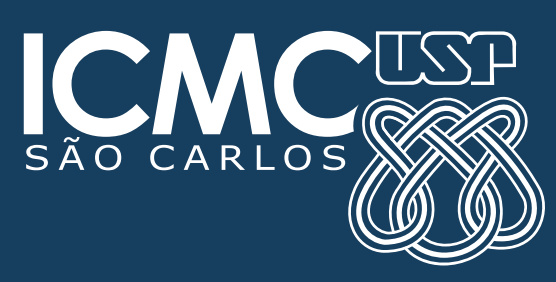

